Noname manuscript No.

(will be inserted by the editor)

\title{
The Multiplicative-Additive Lambek Calculus with Subexponential and Bracket Modalities
}

\author{
Max Kanovich • Stepan Kuznetsov • \\ Andre Scedrov
}

Received: date / Accepted: date

\begin{abstract}
We give a proof-theoretic and algorithmic complexity analysis for systems introduced by Morrill to serve as the core of the CatLog categorial grammar parser. We consider two recent versions of Morrill's calculi, and focus on their fragments including multiplicative (Lambek) connectives, additive conjunction and disjunction, brackets and bracket modalities, and the ! subexponential modality. For both systems, we resolve issues connected with the cut rule and provide necessary modifications, after which we prove admissibility of cut (cut elimination theorem). We also prove algorithmic undecidability for both calculi, and show that categorial grammars based on them can generate arbitrary recursively enumerable languages.
\end{abstract}

Keywords Lambek calculus · categorial grammars · subexponential modalities · bracket modalities · undecidability · cut elimination

M. Kanovich
University College London,
Gowers St., London, U.K.

and National Research University Higher School of Economics,

11 Pokrovsky Blvd., Moscow, Russia

E-mail: m.kanovich@ucl.ac.uk

S. Kuznetsov

Steklov Mathematical Institute of the Russian Academy of Sciences,

8 Gubkina St., Moscow, Russia

and National Research University Higher School of Economics,

11 Pokrovsky Blvd., Moscow, Russia

E-mail: sk@mi-ras.ru

A. Scedrov

University of Pennsylvania,

209 South 33rd St., Philadelphia, PA, U.S.A.

and National Research University Higher School of Economics (until July 2020),

11 Pokrovsky Blvd., Moscow, Russia

E-mail: scedrov@math.upenn.edu 
Mathematics Subject Classification (2010) 03B47 • 03F52 - 03F05 • $03 \mathrm{D} 03 \cdot 03 \mathrm{D} 25$

\section{Linguistic Introduction}

The Lambek calculus (Lambek, 1958) was introduced for mathematical modelling of natural language syntax via categorial grammars. The concept of categorial grammar goes back to ideas of Ajdukiewicz (1935) and Bar-Hillel (1953). The framework of categorial grammars aims to describe natural language by means of logical derivability (see Buszkowski (2003); Carpenter (1997); Morrill (2011); Moot and Retoré (2012) etc). From the modern logical point of view, the calculus of Lambek grammars (the Lambek calculus) is a variant of Girard's linear logic (Girard, 1987) in its non-commutative intuitionistic version (Abrusci, 1990). Nowadays Lambek-style categorial grammars form one framework in a family of closely related formalisms, including combinatory categorial grammars (Steedman, 2000), categorial dependency grammars (Dekhtyar and Dikovsky, 2008), and others.

A categorial grammar assigns logical formulae to lexemes (words) of the language. These formulae are syntactic categories, or types, of these words. In Lambek grammars, types are constructed using three binary connectives, namely two divisions, \and /, and the product, $\cdot$

Following the usual introduction into categorial grammars, we start with the standard example: "John loves Mary." Here "John" and "Mary" receive syntactic type $N$ (noun); "loves," as a transitive verb, is of type $(N \backslash S) / N$. Here $S$ is the syntactic category of grammatically valid sentences. Thus, a transitive verb is handled as something that needs a noun phrase on the left and a noun phrase on the right to become a complete sentence. In the Lambek calculus, $A, A \backslash B$ yields $B$, and so does $B / A, A$ (the complete formulation of the Lambek calculus is presented in Section 2). Thus, $N,(N \backslash S) / N, N \rightarrow S$ is a theorem of the Lambek calculus, which validates "John loves Mary" as a correct sentence.

Lambek grammars are also capable of handling more sophisticated syntactic constructions, in particular, coordination ("and," "or") and some cases of dependent clauses. These cases include examples like "the girl whom John loves" (parsed as $N$ ). Here the most interesting syntactic type is the one for "whom": $(C N \backslash C N) /(S / N)$. The type $C N$ stands for "common noun," i.e., a noun without article. "Whom" takes, as its right argument, an incomplete sentence "John loves," which lacks a noun phrase on the right to become a complete sentence (like "John loves Mary") and is therefore of type $S$ / $N$. The complete analysis of "the girl whom John loves" corresponds to the following theorem of the Lambek calculus:

$$
N / C N, C N,(C N \backslash C N) /(S / N), N,(N \backslash S) / N \rightarrow N .
$$

Coordination between two sentences ( "John loves Mary and Pete loves Ann") is handled by assigning $(S \backslash S) / S$ to "and." 
There are, however, serious limitations of the expressive power of Lambek grammars. Namely, the famous result of Pentus (1993) states that any language described by a Lambek grammar is necessarily context-free. On the other hand, context-freeness of real natural language syntax had been a disputed question in the linguistic community, see Pullum and Gazdar (1982). Finally, Shieber (1985) demonstrated a non-context-free construction in Swiss German. Though examples like Shieber's one may seem exotic, constructing context-free grammars for sophisticated natural phenomena, even if such grammars exist, is practically quite hard. This discrepancy motivates extending and modifying the Lambek calculus in order to obtain more powerful categorial grammar formalisms.

In this paper we consider some of these extensions. In the analysis of linguistic examples, we generally follow Morrill (2011) and later papers by Morrill and his co-authors.

The first extension handles the syntactic phenomenon called medial extraction by means of a subexponential modality allowing permutation. To make it clear what medial extraction is, recall the "the girl whom John loves" example. In this example, the dependent clause "John loves" is a sentence which lacks a noun phrase. Let us call the place where this noun phrase is omitted a gap and denote it by []. A sentence with a gap in the end ("John loves []," cf. "John loves Mary") is of type $S / N$. Symmetrically, a gap in the beginning yields type $N \backslash S$, like for "[ loves Mary" in "the boy who loves Mary." Here "who" receives type $(C N \backslash C N) /(N \backslash S)$. Unfortunately, this does not cover dependent clauses in which the gap is located in the middle of the sentence, i.e., examples like "the girl whom John met [] yesterday." This dependent clause is neither of type $S / N$, nor of type $N \backslash S$.

Medial extraction can be handled by adding a subexponential modality (cf. Kanovich et al. (2019a)), denoted by !, which allows permutation. In general, the Lambek calculus is non-commutative, thus, the order of the words in a sentence matters. For formulae of the form $! A$, however, permutation is allowed, and they can be freely moved. Now the gap gets type $! N$ and can be relocated to an arbitrary place of the dependent clause; the clause in whole receives type $S / ! N$.

Another issue connected to dependent clauses is overgeneration (i.e., wrong judgement of incorrect syntactic structures as valid ones), which arises when dependent clauses and "and"-coordination appear together. An example is * "the girl whom John loves Mary and Pete loves." This is not a correct noun phrase (which is denoted by the asterisk put before it). Unfortunately, "John loves Mary and Pete loves []" is still of type $S / N$ (cf. "John loves Mary and Pete loves Ann" being of type $S$ ), which incorrectly validates our example as a noun phrase. Another example is * "the paper that John saw the person who wrote" (again, we have "John saw the person who wrote []" is of type $S / N$ ).

These wrong derivations can be cut off using the mechanism of brackets (Morrill, 1992, Moortgat, 1996), which introduces controlled non-associativity. Brackets are instantiated by special bracket modalities (see Section 3 for details) and embrace certain parts of the sentence into islands. Islands 
typically include and-coordinated sentences, that-clauses, gerund clauses, etc. Brackets (borders of islands) cannot be penetrated by the permutation rules for $! N$. Thus, the dependent clause with brackets inside is no longer of type $S / ! N$, and the whole wrong derivation gets invalidated.

Finally, we consider a more rare syntactic phenomenon called parasitic extraction, a typical example of which is given by the following noun phrase: "the paper that John signed without reading." In this example we have two gaps: "John signed [] without reading []," and both gaps should be filled with the same object of type $N$ : "John signed the paper without reading the paper." Of course, one can think of examples with three and more gaps, like "the paper that the author of [] signed [] without reading []," and so on. In a series of papers (Morrill, 2014: Morrill and Valentín, 2015a, Morrill, 2017b a, 2018b, 2019), Morrill, with his co-author Valentín, uses several different calculi for handling parasitic extraction. All these approaches, however, use a subexponential modality the contraction rule, which makes proof search problematic and often yields algorithmic undecidability. Generally, contraction is a rule of the form

$$
\frac{\ldots, ! A, \ldots, ! A, \ldots \rightarrow C}{\ldots, ! A, \ldots \rightarrow C}
$$

Morrill and his co-authors, however, suggest more sophisticated versions of contraction, which involve brackets. The general idea of their approaches is as follows: in the situation of parasitic extraction, only one gap lies plainly in the dependent clause; other gaps, which are called parasitic, reside in bracketed subislands of the clause. Moreover, they can get nested. Thus, the contraction rule becomes highly non-standard.

Morrill's systems differ one from another in the rules for !. In this article, we give a logical analysis for two of these systems. One is presented in Morrill and Valentín (2015a); Morrill (2017b) and is closely related to the one of Morrill (2017a). The other one is from Morrill (2019, 2018b). For both systems, we discuss issues connected to cut elimination, and then prove cut elimination for modified versions of these systems. Next, we provide a generic method of encoding semi-Thue systems in extensions of the Lambek calculus with subexponential modalities, and use this method to prove undecidability of the derivability problems for Morrill's systems. We also show that categorial grammars based on these systems generate all recursively enumerable languages. Finally, using methods of Buszkowski (1982), we strengthen these algorithmic results by restricting ourselves to smallest reasonable fragments, which includes only one division, subexponential, brackets, and bracket modalities.

This journal article extends our conference papers in the 21st International Symposium on Fundamentals of Computation Theory, FCT 2017, held in Bordeaux in September 2017 (Kanovich et al., 2017b), and in the 24th Conference on Formal Grammar, FG 2019, held in Riga in August 2019 (Kanovich et al. 2019b). However, here we provide a significant refinement of the results presented in the FCT '17 and FG '19 papers. First, here we consider the system with additive connectives. This makes cut elimination results stronger. Second, besides undecidability, we also show that categorial grammars based on each of 
the calculi in question generate all recursively enumerable languages, not just one $\Sigma_{1}^{0}$-hard one (Section 10. Third, using a variant of Buszkowski's translation (Buszkowski, 1982), we establish undecidability even for the one-division fragments of the calculi in question (Section 11).

In comparison with a series of our papers on the Lambek calculus and non-commutative linear logic with subexponential modalities (Kanovich et al. 2016a, 2020, 2016b, 2019a, 2018), the principal difference of this paper is the presence of brackets and bracket modalities. Contraction rules used by Morrill in the bracketed calculi essentially interact with brackets and become disfunctional in the bracket-free fragment. Undecidability results, on their turn, rely on contraction. Thus, they should be proved for calculi with brackets independently from the bracket-free case.

On the other side, two papers on the bracketed Lambek calculus (Kanovich et al. 2017a; Morrill et al., 2018) do not deal with the subexponential modality (!), and feature effective algorithms instead of undecidability results.

\section{The Multiplicative-Additive Lambek Calculus with Exponential/Relevant Modality}

We start with more traditional calculi without brackets and bracket modalities, namely, the multiplicative-additive Lambek calculus extended with a (sub)exponential modality.

Formulae of the calculi we are going to define in this section are constructed from a countable set Var of variables and the unit constant 1 using five binary connectives: · (product, or multiplicative conjunction), \(left division), / (right division), $\wedge$ (additive conjunction), and $\vee$ (additive disjuncton), and one unary connective, ! (exponential). Sequents are expressions of the form $\Pi \rightarrow A$, where $A$ is a formula, and $\Pi$ is a finite linearly ordered sequence of formulae. Notice that these calculi are in general non-commutative, $\Pi$ is a sequence, not a set or multiset.

The first calculus we consider is ${ }^{\mathbf{r}} \mathbf{M} \mathbf{A L C} \mathbf{C}^{*}$, the multiplicative-additive Lambek calculus extended with a relevant subexponential modality (r stands for "relevant," see below). The axioms of ! ${ }^{\mathbf{r}} \mathbf{M A L C}{ }^{*}$ are sequents of the form $A \rightarrow A$ and $\Lambda \rightarrow \mathbf{1}$, and the rules of inference are as follows:

$$
\begin{gathered}
\frac{A, \Pi \rightarrow B}{\Pi \rightarrow A \backslash B} \backslash R \quad \frac{\Pi \rightarrow A \quad \Delta_{1}, B, \Delta_{2} \rightarrow C}{\Delta_{1}, \Pi, A \backslash B, \Delta_{2} \rightarrow C} \backslash L \\
\frac{\Pi, A \rightarrow B}{\Pi \rightarrow B / A} / R \quad \frac{\Pi \rightarrow A \quad \Delta_{1}, B, \Delta_{2} \rightarrow C}{\Delta_{1}, B / A, \Pi, \Delta_{2} \rightarrow C} / L \\
\frac{\Gamma \rightarrow A \quad \Delta \rightarrow B}{\Gamma, \Delta \rightarrow A \cdot B} \cdot R \\
\frac{\Delta_{1}, A, B, \Delta_{2} \rightarrow C}{\Delta_{1}, A \cdot B, \Delta_{2} \rightarrow C} \cdot L \quad \frac{\Delta_{1}, \Delta_{2} \rightarrow C}{\Delta_{1}, \mathbf{1}, \Delta_{2} \rightarrow C} \mathbf{1} \\
\frac{\Pi \rightarrow A_{1} \Pi \rightarrow A_{2}}{\Pi \rightarrow A_{1} \wedge A_{2}} \wedge R \quad \frac{\Delta_{1}, A_{i}, \Delta_{2} \rightarrow C}{\Delta_{1}, A_{1} \wedge A_{2}, \Delta_{2} \rightarrow C} \wedge L_{i}, i=1,2
\end{gathered}
$$




$$
\begin{gathered}
\frac{\Pi \rightarrow A_{i}}{\Pi \rightarrow A_{1} \vee A_{2}} \vee R_{i}, i=1,2 \quad \frac{\Delta_{1}, A_{1}, \Delta_{2} \rightarrow C \quad \Delta_{1}, A_{2}, \Delta_{2} \rightarrow C}{\Delta_{1}, A_{1} \vee A_{2}, \Delta_{2} \rightarrow C} \vee L \\
\frac{! A_{1}, \ldots, ! A_{n} \rightarrow B}{! A_{1}, \ldots, ! A_{n} \rightarrow ! B} ! R \quad \frac{\Delta_{1}, A, \Delta_{2} \rightarrow C}{\Delta_{1}, ! A, \Delta_{2} \rightarrow C} ! L \\
\frac{\Delta_{1}, ! A, \Phi, \Delta_{2} \rightarrow C}{\Delta_{1}, \Phi, ! A, \Delta_{2} \rightarrow C} ! P_{1} \quad \frac{\Delta_{1}, \Phi, ! A, \Delta_{2} \rightarrow C}{\Delta_{1}, ! A, \Phi, \Delta_{2} \rightarrow C} ! P_{2} \\
\frac{\Delta_{1}, ! A, ! A, \Delta_{2} \rightarrow C}{\Delta_{1}, ! A, \Delta_{2} \rightarrow C} ! C \\
\frac{\Pi \rightarrow A}{\Delta_{1}, A, \Delta_{2} \rightarrow C} \\
\text { cut }
\end{gathered}
$$

Notice that, from the proof-theoretic point of view, it is better to use, instead of (contr), the following non-local contraction rules (Kanovich et al. 2019a):

$$
\frac{\Delta_{1}, ! A, \Phi, ! A, \Delta_{2} \rightarrow C}{\Delta_{1}, \Phi, ! A, \Delta_{2} \rightarrow C} ! N C_{1} \quad \frac{\Delta_{1}, ! A, \Phi, ! A, \Delta_{2} \rightarrow C}{\Delta_{1}, ! A, \Phi, \Delta_{2} \rightarrow C} ! N C_{2}
$$

In the presence of $! P_{1,2}$, however, ! $C$ has the same power as $! N C_{1,2}$.

The ! modality here is called "relevant," since it allows contraction and permutation, but not weakening, like in relevant logic.

The second system without brackets is !MALC* ${ }^{*}$, the multiplicative-additive Lambek calculus extended with a full-power exponential modality. It is obtained from ' $^{\mathrm{r}} \mathbf{M} \mathbf{A L C}{ }^{*}$ by adding the lacking structural rule for !, namely weakening:

$$
\frac{\Gamma, \Delta \rightarrow C}{\Gamma, ! A, \Delta \rightarrow C} ! W
$$

Both !' $\mathbf{M A L C}{ }^{*}$ and :MALC $^{*}$ are particular cases of $\mathbf{S M A L C} \mathbf{C}_{\Sigma}$, the multiplicative-additive Lambek calculus extended with an arbitrary family of subexponentials $\Sigma$, considered by Kanovich et al. (2019a). In that paper it is shown that these calculi enjoy cut elimination and that the derivability problems for these calculi are undecidable.

Cut elimination yields the subformula property (each formula occurring in the cut-free derivation is a subformula of the goal sequent) and thus conservativity of elementary fragments. Namely, if one wants to derive only sequents that include formulae with a restricted set of connectives, it is sufficient just to restrict the set of rules of the calculus to this set of connectives. For convenience, we use a shorter notation, $!^{\mathrm{r}} \mathbf{L}^{*}$ and $! \mathbf{L}^{*}$, for the fragments without additive connectives $\left(\vee\right.$ and $\wedge$ ) of ' $^{\mathbf{r}} \mathbf{M A L C}{ }^{*}$ and !MALC ${ }^{*}$ respectively.

Let us formally define the notion of categorial grammar based on a noncommutative intuitionistic-style sequent calculus $\mathcal{L}$ without brackets, like the systems ! ${ }^{\mathbf{r}} \mathbf{M A L C} \mathbf{C}^{*}$ and !MALC* defined above. 
Definition 1 An $\mathcal{L}$-grammar is a triple $\mathcal{G}=\langle\Sigma, \triangleright, H\rangle$, where $\Sigma$ is a finite alphabet, $H$ is a formula, and $\triangleright$ is a finite binary correspondence between letters of $\Sigma$ and formulae (called lexicon). A word $w=a_{1} \ldots a_{n}$ over $\Sigma$ is accepted by $\mathcal{G}$ if there exist formulae $A_{1}, \ldots, A_{n}$ such that $a_{i} \triangleright A_{i}(i=1, \ldots, n)$ and the sequent $A_{1}, \ldots, A_{n} \rightarrow H$ is derivable in $\mathcal{L}$. The language generated, or recognised, by $\mathcal{G}$ consists of all words recognised by $\mathcal{G}$.

The system with weakening, !MALC* ${ }^{*}$, has not so much to do with linguistic applications, but is interesting from the logical point of view. In particular, we use it as an intermediate calculus in our undecidability proofs (Sections 9 and 11).

The system with a relevant modality, ! ${ }^{\mathrm{r}} \mathbf{M A L C}{ }^{*}$, supports analysis of many cases of extraction from dependent clauses, including parasitic extraction. For example, "the paper that John signed without reading" is analysed as follows. First, we define the necessary fragment of the lexicon:

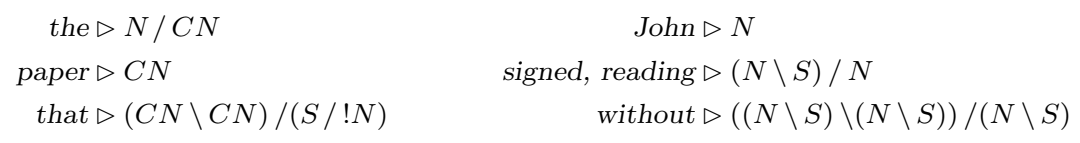

Here $N$ stands for "noun phrase," $C N$ states for "common noun" (without an article), and $S$ stands for "sentence." Next, we derive the sequent

$$
\begin{aligned}
& N / C N, C N,(C N \backslash C N) /(S / ! N), N,(N \backslash S) / N, \\
& ((N \backslash S) \backslash(N \backslash S)) /(N \backslash S),(N \backslash S) / N \rightarrow N
\end{aligned}
$$

in ${ }^{\mathbf{r}}$ MALC*, as shown on Figure 1 .

Without brackets, however, categorial grammars based on ! $^{\mathbf{M}}$ MALC ${ }^{*}$ suffer from overgeneration, parsing ungrammatical phrases like * "the girl whom John loves Mary and Pete loves" (see Introduction). In the next section, we introduce systems including both brackets and a restricted subexponential, developed by Morrill in a series of papers.

\section{Morrill's Calculi with Brackets and Subexponential}

In this section we describe extensions of the Lambek calculus, which include both brackets (and bracket modalities which control them) and a subexponential, which interacts with brackets in an intricate way.

In his papers, Morrill (sometimes with his co-author Valentín) introduces different variants of his calculus - the difference is in the most interesting rule, contraction. We consider two of Morrill's calculi, and denote these calculi by $!_{b}^{2015} \mathrm{MALC}^{*} \mathrm{~b}(\mathrm{st})$ and $!_{\mathrm{b}}^{\mathbf{2 0 1 8}} \mathrm{MALC}^{*} \mathrm{~b}(\mathrm{st})$, by the year of first publication. In this notation, "(st)" means the presence of stoups, the $\mathbf{b}$ on the right stands for "brackets," and $!_{\mathrm{b}}$ means that the subexponential ! interacts with the bracketing structure.

The $!_{\mathrm{b}}^{\mathbf{2 0 1 5}} \mathbf{M A L C} \mathbf{C}^{*} \mathbf{b}(\mathrm{st})$ system, in its version without stoups, appears in Morrill and Valentín (2015a), and then in Morrill (2017b) (Morrill (2017a) 


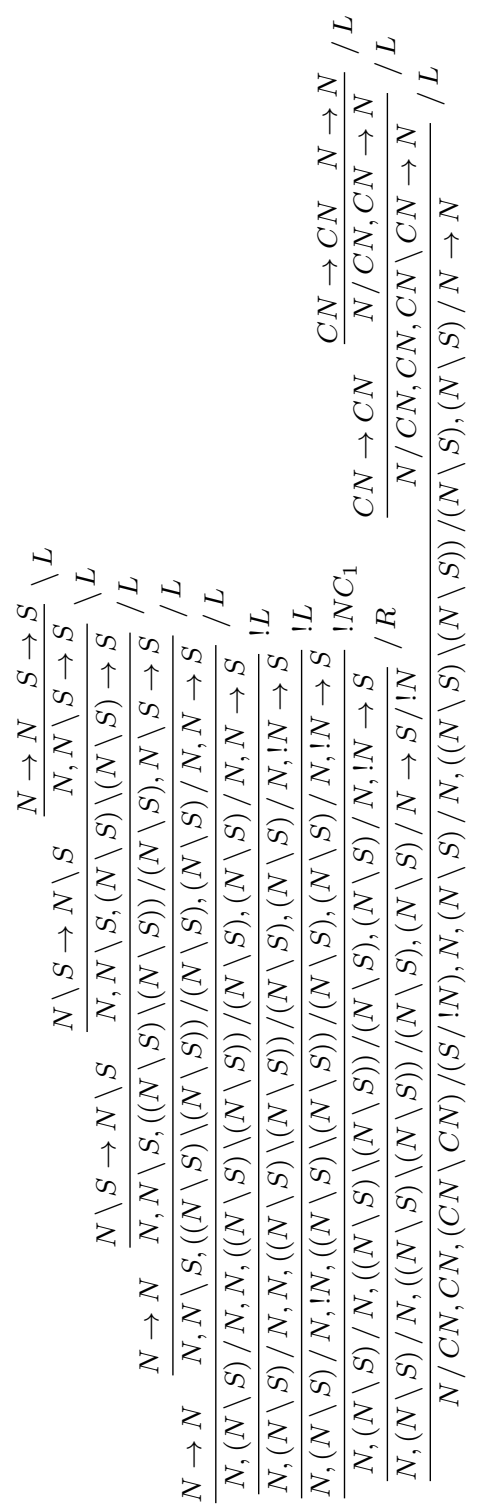

Fig. 1 Derivation for "the paper that John signed without reading" in !r MALC* (like (Morrill 2019, Fig. 24), but with brackets and bracket modalities removed)

features a slightly different version of this system). The $!_{\mathbf{b}}^{2018} \mathbf{M A L C} \mathbf{C}^{*} \mathbf{b}(\mathrm{st})$ system appears in Morrill's recent papers (Morrill, 2018b, 2019); however, essentially here Morrill returns to an older formulation of the bracket-aware contraction rule (Morrill, 2011, 2014). 
Morrill's systems are quite involved, including up to 45 connectives. In this article we consider their simpler fragments, including multiplicative and additive Lambek connectives $(\backslash, /, \cdot, \mathbf{1}, \vee, \wedge)$, brackets and bracket modalities $\left(\langle\rangle\right.$ and []$\left.^{-1}\right)$, and the subexponential !. Since all Morrill's systems do not include cut as a rule, these fragments are conservative inside the bigger systems, and our undecidability results also work for the latter. (The question of admissibility of cut in Morrill's system is more subtle, and we discuss it later on.)

Before going forward, let us notice that full Morrill's systems also include Kleene star, axiomatised by means of an $\omega$-rule (Morrill calls it "existential exponential" and denotes by "?"). In the presence of Kleene star, the Lambek calculus is known to be at least $\Pi_{1}^{0}$-hard (Buszkowski and Palka, 2008 Kuznetsov, 2017), if the $\omega$-rule is used, and at least $\Sigma_{1}^{0}$-hard (Kuznetsov 2019), if the Kleene star is axiomatised by means of induction axioms. In both cases, this means undecidability. Moreover, in the view of Kozen's results on complexity of Horn theories of Kleene algebras (Kozen, 2002), the complexity of a system with both Kleene star (with an $\omega$-rule) and a subexponential modality allowing contraction is likely to rise up to $\Pi_{1}^{1}$-completeness. Morrill, however, emphasizes the fact that in formulae used in categorial grammars designed for real languages the Kleene star never occurs with positive polarity. Thus, the $\omega$-rule is never used, and the Kleene star does not incur problems with decidability. Thus, the only possible source of undecidability is the specific contraction rule for the subexponential. We consider fragments of Morrill's systems with this rule, which are sufficient to show undecidability.

The syntax and metasyntax of sequents in Morrill's systems (in particular, their fragments considered throughout this article) is more involved, if compared to the calculi without brackets. First, in the antecedents we now have brackets which operate along with the structural comma (a metasyntactic correspondent of the product connective), introducing partial non-associativity. Second, in order to avoid superfluous usage of permutation rules for !-formulae and to facilitate proof search, in his systems Morrill groups the !-formulae to specifically designated commutative areas in the sequent. Using the terminology of Girard (1991, 1993), Morrill calles these areas stoups. Morrill's calculi, both technically and ideologically, are close to the sequent system by Hodas and Miller (1994). In that system, antecedents are split into two zones, $\zeta ; \Delta$, where $\zeta$ is the intuitionistic zone (formulae there are allowed to contract and weaken) and $\Delta$ is the linear one. In Morrill's terms, $\zeta$ is the stoup. Morrill's rules are more complicated, because of non-commutativity of the system in general, and also partial non-associativity introduced by brackets. Introducing the stoups, in fact, is the first step towards a focused proof system (Andreoli 1992, Morrill and Valentín, 2015b; Kanovich et al., 2018). Since permutations for !-formulae cannot penetrate brackets, each pair of brackets has its own stoup.

Let us define the syntax formally. Formulae will be built from variables (primitive types) $p, q, \ldots$ and the multiplicative unit constant $\mathbf{1}$ using three binary operations: \(left division), / (right division), · (product), and three 
unary operations: \langle\rangle and []$^{-1}$ (bracket modalities) and ! (subexponential). Sequents (in Morrill's terminology, h-sequents) are expressions of the form $\Xi \Rightarrow A$, where $A$ is a formula and $\Xi$ is a complex metasyntactic structure which we call meta-formula (Morrill calls them zones). Meta-formulae are built from formulae using comma and brackets; also formulae which are intended to be marked by the subexponential !, which allows permutation, are placed into stoups. Following Morrill (2019), we define the notion of meta-formula along with two auxiliary notions, stoup and tree term, simultaneously.

- A stoup is a multiset of formulae: $\zeta=\left\{A_{1}, \ldots, A_{n}\right\}$. A stoup could be empty, the empty stoup is denoted by $\varnothing$.

- A tree term is either a formula or a bracketed expression of the form $[\Xi]$, where $\Xi$ is a meta-formula.

- A meta-formula is an expression of the form $\zeta ; \Gamma$, where $\zeta$ is a stoup and $\Gamma$ is a linearly ordered sequence of tree terms. Here $\Gamma$ could also be empty; the empty sequence is denoted by $\Lambda$.

We use comma both for concatenation of tree term sequences and for multiset union of stoups (Morrill uses $\uplus$ for the latter). Moreover, for adding one formula into a stoup we write $\zeta, A$ instead of $\zeta,\{A\}$. Empty stoups are omitted: instead of $\varnothing ; \Gamma$ we write just $\Gamma$.

Let us first formulate the rules which do not operate!, since these rules are the same in all Morrill's systems.

$$
\begin{aligned}
& \overline{A \rightarrow A} \text { id } \\
& \frac{\zeta_{1} ; \Gamma \rightarrow B \quad \Xi\left(\zeta_{2} ; \Delta_{1}, C, \Delta_{2}\right) \rightarrow D}{\Xi\left(\zeta_{1}, \zeta_{2} ; \Delta_{1}, C / B, \Gamma, \Delta_{2}\right) \rightarrow D} / L \quad \frac{\zeta ; \Gamma, B \rightarrow C}{\zeta ; \Gamma \rightarrow C / B} / R \\
& \frac{\zeta_{1} ; \Gamma \rightarrow A \quad \Xi\left(\zeta_{2} ; \Delta_{1}, C, \Delta_{2}\right) \rightarrow D}{\Xi\left(\zeta_{1}, \zeta_{2} ; \Delta_{1}, \Gamma, A \backslash C, \Delta_{2}\right) \rightarrow D} \backslash L \quad \frac{\zeta ; A, \Gamma \rightarrow C}{\zeta ; \Gamma \rightarrow A \backslash C} \backslash R \\
& \frac{\Xi\left(\zeta ; \Delta_{1}, A, B, \Delta_{2}\right) \rightarrow D}{\Xi\left(\zeta ; \Delta_{1}, A \cdot B, \Delta_{2}\right) \rightarrow D} \cdot L \quad \frac{\zeta_{1} ; \Delta \rightarrow A \quad \zeta_{2} ; \Gamma \rightarrow B}{\zeta_{1}, \zeta_{2} ; \Delta, \Gamma \rightarrow A \cdot B} \cdot R \\
& \frac{\Xi \rightarrow A_{i}}{\Xi \rightarrow A_{1} \vee A_{2}} \vee R_{i} i=1,2 \quad \frac{\Xi\left(\zeta ; \Delta_{1}, \Delta_{2}\right) \rightarrow A}{\Xi\left(\zeta ; \Delta_{1}, \mathbf{1}, \Delta_{2}\right) \rightarrow A} \mathbf{1} L \\
& \frac{\Xi\left(\zeta ; \Delta_{1}, A_{1}, \Delta_{2}\right) \rightarrow C \quad \Xi\left(\zeta ; \Delta_{1}, A_{2}, \Delta_{2}\right) \rightarrow C}{\Xi\left(\zeta ; \Delta_{1}, A_{1} \vee A_{2}, \Delta_{2}\right) \rightarrow C} \vee L \quad \overline{\Lambda \rightarrow \mathbf{1}} \mathbf{1} R \\
& \frac{\Xi\left(\zeta ; \Delta_{1}, A_{j}, \Delta_{2}\right) \rightarrow C}{\Xi\left(\zeta ; \Delta_{1}, A_{1} \wedge A_{2}, \Delta_{2}\right) \rightarrow C} \wedge L_{j} j=1,2 \quad \frac{\Xi \rightarrow A_{1} \quad \Xi \wedge A_{2}}{\Xi \rightarrow A_{1} \wedge A_{2}} \wedge R
\end{aligned}
$$




$$
\begin{array}{cc}
\frac{\Xi\left(\zeta ; \Delta_{1}, A, \Delta_{2}\right) \rightarrow B}{\Xi\left(\zeta ; \Delta_{1},\left[[]^{-1} A\right], \Delta_{2}\right) \rightarrow B}[]^{-1} L & \frac{[\Xi] \rightarrow A}{\Xi \rightarrow[]^{-1} A}[]^{-1} R \\
\frac{\Xi\left(\zeta ; \Delta_{1},[A], \Delta_{2}\right) \rightarrow B}{\Xi\left(\zeta ; \Delta_{1},\langle\rangle A, \Delta_{2}\right) \rightarrow B}\langle\rangle L & \frac{\Xi \rightarrow A}{[\Xi] \rightarrow\langle\rangle A}\langle\rangle R
\end{array}
$$

The two calculi, $!_{\mathbf{b}}^{\mathbf{2 0 1 5}} \mathbf{M A L C} \mathbf{C}^{*} \mathbf{b}(\mathrm{st})$ and $!_{\mathbf{b}}^{\mathbf{2 0 1 8}} \mathbf{M} \mathbf{A L C} \mathbf{L}(\mathrm{st})$, also share two rules for !:

$$
\frac{\Xi\left(\zeta, A ; \Gamma_{1}, \Gamma_{2}\right) \rightarrow B}{\Xi\left(\zeta ; \Gamma_{1}, ! A, \Gamma_{2}\right) \rightarrow B} ! L \quad \frac{\Xi\left(\zeta ; \Gamma_{1}, A, \Gamma_{2}\right) \rightarrow B}{\Xi\left(\zeta, A ; \Gamma_{1}, \Gamma_{2}\right) \rightarrow B} ! P
$$

However, the $! R$ rule and, most importantly, the contraction rule $! C$ are different. In the "older" system $!_{\mathbf{b}}^{\mathbf{2 0 1 5}} \mathbf{M} \mathbf{A L C} \mathbf{C}^{*} \mathbf{b}(\mathrm{st})$ they are formulated as follows:

$$
\frac{\zeta ; \Lambda \rightarrow B}{\zeta ; \Lambda \rightarrow ! B} ! R \quad \frac{\Xi\left(\zeta_{1}, \zeta_{2} ; \Gamma_{1},\left[\zeta_{2} ; \Gamma_{2}\right], \Gamma_{3}\right) \rightarrow B}{\Xi\left(\zeta_{1}, \zeta_{2} ; \Gamma_{1}, \Gamma_{2}, \Gamma_{3}\right) \rightarrow B} ! C, \zeta_{2} \neq \varnothing
$$

The "newer" system $!_{\mathbf{b}}^{\mathbf{2 0 1 8}} \mathbf{M A L C}{ }^{*} \mathbf{b}($ st) uses the following formulation of $! R$ and $! C$ :

$$
\frac{! A \rightarrow B}{! A \rightarrow ! B} ! R \quad \frac{\Xi\left(\zeta, A ; \Gamma_{1},\left[A ; \Gamma_{2}\right], \Gamma_{3}\right) \rightarrow B}{\Xi\left(\zeta, A ; \Gamma_{1},\left[\left[\Gamma_{2}\right]\right], \Gamma_{3}\right) \rightarrow B} ! C
$$

As noticed above, in the absence of cut we can easily formulate fragments of $!_{\mathrm{b}}^{\mathbf{2 0 1 5}} \mathbf{M A L C} \mathbf{C}^{*} \mathbf{b}(\mathrm{st})$ and $!_{\mathrm{b}}^{\mathbf{2 0 1 8}} \mathbf{M A L C} \mathbf{C}^{*} \mathbf{b}(\mathrm{st})$ without additive connectives: one just removes the corresponding rules $\left(\vee L, \vee R_{1,2}, \wedge L_{1,2}, \wedge R\right)$. In the notations, we just replace "MALC" with "L": $!_{\mathbf{b}}^{\mathbf{2 0 1 5}} \mathbf{L}^{*} \mathbf{b}(\mathrm{st}), !_{\mathbf{b}}^{\mathbf{2 0 1 8}} \mathbf{L}^{*} \mathbf{b}(\mathrm{st})$. In the following sections we use the same naming convention: if a calculus' name includes "MALC," then replacing it with "L" gives a name for the fragment of this calculus without additive connectives.

For calculi with brackets, defining recognition of words in categorial grammars is trickier. One can keep the definition from Section 2 and say that $w=a_{1} \ldots a_{n}$ is accepted by the grammar if $A_{1}, \ldots, A_{n} \rightarrow H$ is derivable, for some $A_{i}$ such that $a_{i} \triangleright A_{i}(i=1, \ldots, n)$. Notice that this sequent does not include brackets, but may include bracket modalities, \langle\rangle and []$^{-1}$. Thus, brackets could appear inside the derivation. This notion of recognition is called $s$-recognition (Jäger, 2003).

Linguistic applications, however, suggest another notion of recognition for Lambek grammars with brackets, called t-recognition. A word $w=a_{1} \ldots a_{n}$ is t-accepted by an grammar $\mathcal{G}$ if the sequent $\Pi \rightarrow H$ is derivable for some $\Pi$ such that if one removes all brackets (but not bracket modalities!) from $\Pi$, it yields $A_{1}, \ldots, A_{n}$, where $a_{i} \triangleright A_{i}(i=1, \ldots, n)$. In other words, a word is accepted if there corresponding sequent is derivable for some bracketing $\Pi$.

In the implementation of Morrill's bracketed calculi in the CatLog parser, the bracket structure on $A_{1}, \ldots, A_{n}$ is requested from the user as part of input data (Morrill, 2018a). There is an ongoing project of implementing automatic 
guessing of the correct bracket structure (so-called bracket induction); at the present time, there exists such an algorithm for the fragment with only multiplicative connectives and bracket modalities, without subexponential (Morrill et al. 2018).

As an example, we analyse the phrase "the paper that John signed without reading" using $!_{\mathbf{b}}^{\mathbf{2 0 1 8}} \mathbf{M A L C} \mathbf{C}^{*} \mathbf{b}(\mathrm{st})$. Our analysis is a simplification of the one of Morrill (2019). In comparison with the analysis in Section 2 (Figure 1), here we take care of the bracketed domains, which cannot be penetrated by associativity of product or permutations of !-formulae. Also notice that the contraction rule here implements parasitic extension in the following sense: applying contraction to $! N$ (actually, to $N$ located in the stoup) instantiates a secondary (parasitic) copy of $! N$ into an island. In order to prevent reusage of islands for parasitic extraction, the island transforms from a strong (doublebracketed) to a weak (single-bracketed) one. The lexicon now is as follows (if compared to the one in Section 2 the types here are augmented with bracket modalities):
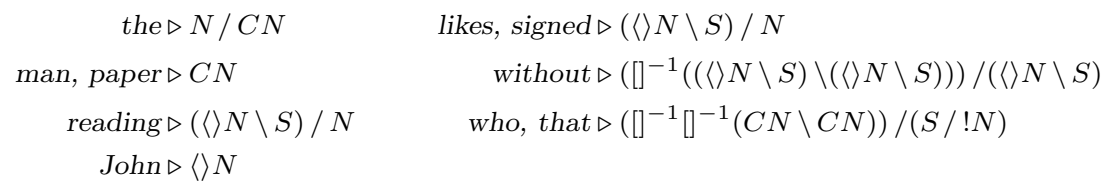

Before parsing, we have to impose the right bracket structure on our phrase. This is done as follows: "the paper [[that [John] signed [[without reading]] ]]." Indeed, in Morrill's CatLog categorial grammar the subject group and the without-clause form islands, and the that-clause forms a strong island, embraced by double brackets. Moreover, we also have to double-bracket our without-clause (make it a "strong island"), since it will be used for parasitic extraction.

Now the sequent we have to derive in $!_{\mathbf{b}}^{\mathbf{2 0 1 8}} \mathbf{M A L C} \mathbf{C}^{*} \mathbf{b}(\mathrm{st})$ is as follows:

$$
\begin{aligned}
N / C N,\left[\left[\left([]^{-1}[]^{-1}(C N \backslash C N)\right) /(S / ! N),[N],(\langle\rangle N \backslash S) / N,\right.\right. \\
{\left.\left.\left[\left[\left([]^{-1}((\langle\rangle N \backslash S) \backslash(\langle\rangle N \backslash S))\right) /(\langle\rangle N \backslash S),(\langle\rangle N \backslash S) / N\right]\right]\right]\right] \rightarrow N }
\end{aligned}
$$

The derivation is presented on Figure 2 .

\section{Issues with Cut Elimination}

Cut elimination is one of the standard logical properties which is expected from a reasonable Gentzen-style sequent calculus. Since in the systems discussed in this article cut is not included as an official rule, the question of cut elimination appears as the question of the admissibility of cut. From the linguistic perspective, cut supports the principle of compositionality: once we have proved that a phrase has syntactic type, say, $N P$, we can use it at any place where a noun phrase is allowed. 


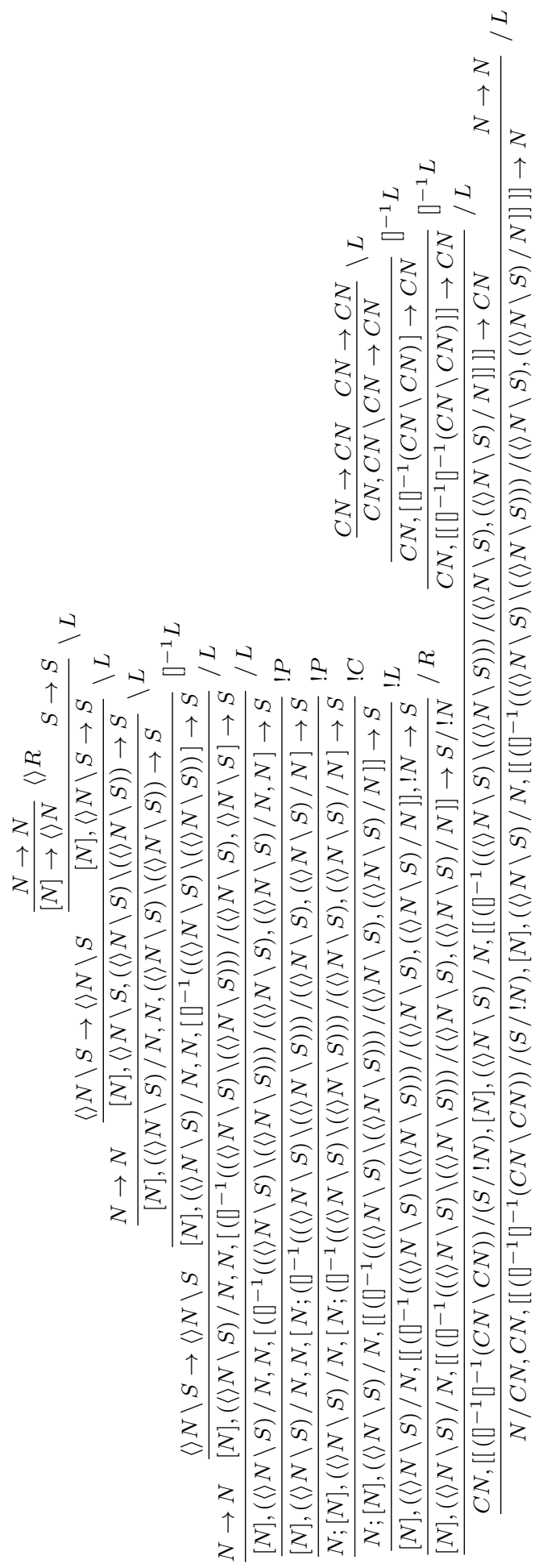

Fig. 2 Derivation for "the paper that John signed without reading" in $\mathbf{~}_{\mathbf{b}}^{\mathbf{2 0 1 8}} \mathbf{M} \mathbf{M L C} \mathbf{\text { }} \mathbf{b}(\mathrm{st})$ (cf. Morrill (2019 Fig. 24)) 
Morrill (2019) mentions a semantic approach to prove admissibility of cut in $!_{\mathbf{b}}^{2018} \mathbf{M A L C} \mathbf{L}^{*} \mathbf{b}($ st) as an ongoing work by O. Valentín. In this paper, we wish to pursue the more traditional syntactic approach for cut elimination, both in $!_{\mathrm{b}}^{\mathbf{2 0 1 5}} \mathbf{M A L C}{ }^{*} \mathbf{b}(\mathrm{st})$ and $!_{\mathrm{b}}^{\mathbf{2 0 1 8}} \mathbf{M A L C}{ }^{*} \mathbf{b}(\mathrm{st})$.

Unfortunately, Morrill's systems, as formulated above (Section 3), fail to enjoy cut elimination (cut admissibility). For $\mathbf{~}_{\mathbf{b}}^{\mathbf{2 0 1 8}} \mathbf{M A L C} \mathbf{M}^{*} \mathbf{b}(\mathrm{st})$, the counterexample is $! p, q \rightarrow q \cdot ! p$. This sequent expresses the natural property that !-formulae commute with arbitrary formulae, and it is derivable using cut:

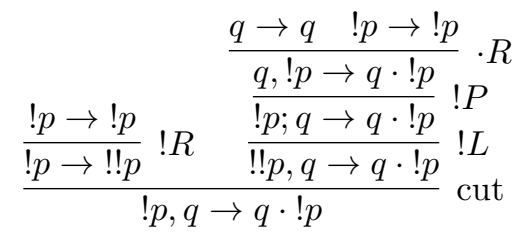

However, no cut-free derivation is available. Indeed, the lowermost rule of such a derivation should be either $R$ or $! L$. The former is impossible, since neither $\Lambda \rightarrow q$, nor $! p \rightarrow q$, nor $! p, q \rightarrow q$ is derivable. In the latter case, we get $p ; q \rightarrow q \cdot ! p$ and again have two possibilities: $R$ or $! P$. For $\cdot R$, the only possible way of splitting could be $q \rightarrow q$ and $p ; \Lambda \rightarrow ! p$. The latter, however, is counter-intuitively not derivable (though $p$ in stoup should mean $! p$ ): one cannot immediately apply $! R$, and applying $! P$ gives $p \rightarrow ! p$. Applying $! P$ would give either $p, q \rightarrow q \cdot p$ or $q, p \rightarrow q \cdot ! p$, none of which is derivable. Notice that the proof search here is finite, since the contraction rule could not be used in the absence of brackets.

Thus, we have to modify $!_{\mathbf{b}}^{\mathbf{2 0 1 8}} \mathbf{M} \mathbf{A L C} \mathbf{C}^{*} \mathbf{b}(\mathrm{st})$ in order to restore the cut elimination property. We do this by replacing $! R$ and $! C$ with the following rules:

$$
\frac{A ; \Lambda \rightarrow B}{A ; \Lambda \rightarrow ! B} ! R^{\prime} \quad \frac{\Xi\left(\zeta, A ; \Gamma_{1},\left[\zeta^{\prime}, A ; \Gamma_{2}\right], \Gamma_{3}\right) \rightarrow B}{\Xi\left(\zeta, A ; \Gamma_{1},\left[\left[\zeta^{\prime} ; \Gamma_{2}\right]\right], \Gamma_{3}\right) \rightarrow B} ! C^{\prime}
$$

Notice that $! R^{\prime}$ corresponds to the $! R$ rule of Morrill's $!_{\mathbf{b}}^{\mathbf{2 0 1 5}} \mathbf{M A L C}{ }^{*} \mathbf{b}(\mathrm{st})$. The only difference is that here the stoup should include exactly one formula.

We denote the modified calculi by $!_{\mathbf{b}}^{\mathbf{2 0 1 8}} \mathbf{M A L C} \mathbf{M} \mathbf{b}(\mathrm{st})^{\prime}$.

In what follows, we shall show (Theorem 1 that $!_{\mathbf{b}}^{\mathbf{2 0 1 8}} \mathbf{M A L C} \mathbf{M}^{*} \mathbf{b}(\mathrm{st})^{\prime}$ admits the cut rule in the following stoup-aware form:

$$
\frac{\xi ; \Pi \rightarrow A \quad \Xi\left(\zeta ; \Gamma_{1}, A, \Gamma_{2}\right) \rightarrow C}{\Xi\left(\xi, \zeta ; \Gamma_{1}, \Pi, \Gamma_{2}\right) \rightarrow C} \mathrm{cut}
$$

Using cut and the left rules for !, one can derive the old $! R$ rule from the new $! R^{\prime}$ one:

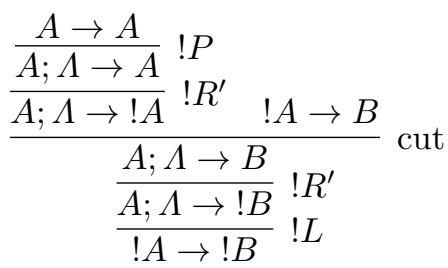


As for $! C^{\prime}$, the old rule $! C$ is just its particular case, for $\zeta^{\prime}=\varnothing$. Thus, $!_{\mathrm{b}}^{\mathbf{2 0 1 8}} \mathrm{MALC}{ }^{*} \mathbf{b}(\mathrm{st})^{\prime}$ is an extension of $!_{\mathrm{b}}^{\mathbf{2 0 1 8}} \mathbf{M A L C} \mathbf{M}(\mathrm{st})$.

For $!_{\mathbf{b}}^{\mathbf{2 0 1 5}} \mathbf{M A L C}{ }^{*} \mathbf{b}($ st), problems come from the non-emptiness restriction imposed on the contraction rule. The $! C$ rule in $!_{\mathbf{b}}^{\mathbf{2 0 1 5}} \mathbf{M A L C} \mathbf{C}^{*} \mathbf{b}(\mathrm{st})$ is formulated in the "multi-contraction" form, allowing to contract several formulae in the stoup at once. However, it should contract at least one formula. This constraint can be easily violated by cut with $\Lambda \rightarrow ! 1$ (which is derivable in $!_{\mathbf{b}}^{\mathbf{2 0 1 5}} \mathbf{M A L C} \mathbf{C}^{*} \mathbf{b}(\mathrm{st})$ ). In systems without brackets this would not be an issue, since in such systems contraction of zero formulae does nothing. In $!_{\mathbf{b}}^{\mathbf{2 0 1 5}} \mathbf{M A L C} \mathbf{C}^{*} \mathbf{b}(\mathrm{st})$, however, !C operates brackets, so such a "zerocontraction" would violate bracket discipline.

The concrete counter-example is $q \rightarrow\langle\rangle q$. This sequent clearly has no cutfree derivation, but can be derived using cut:

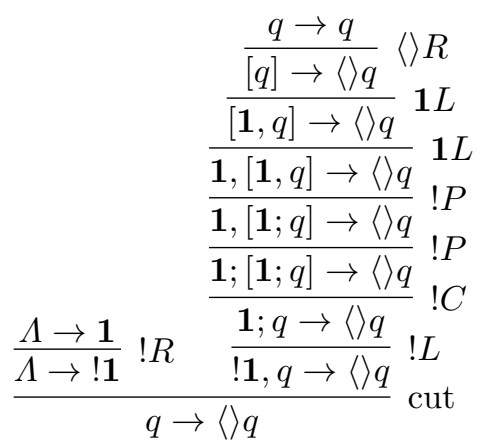

We modify $!_{\mathbf{b}}^{\mathbf{2 0 1 5}} \mathbf{M A L C} \mathbf{C}^{*} \mathbf{b}$ (st) in the following way, yielding the system $!_{\mathrm{b}}^{\mathbf{2 0 1 5}} \mathrm{MALC}^{*} \mathrm{~b}(\mathrm{st})^{\prime}$ :

$$
\frac{\zeta ; \Lambda \rightarrow B}{\zeta ; \Lambda \rightarrow ! B} ! R^{\prime}, \zeta \neq \varnothing \quad \frac{\Xi\left(\zeta_{1}, \zeta_{2} ; \Gamma_{1},\left[\zeta^{\prime}, \zeta_{2} ; \Gamma_{2}\right], \Gamma_{3}\right) \rightarrow C}{\Xi\left(\zeta_{1}, \zeta_{2}, \zeta^{\prime} ; \Gamma_{1}, \Gamma_{2}, \Gamma_{3}\right) \rightarrow C} ! C^{\prime}, \zeta_{2} \neq \varnothing
$$

Theorem 2 establishes cut admissiblity in $!_{\mathbf{b}}^{\mathbf{2 0 1 5}} \mathbf{M A L C} \mathbf{M}(\mathrm{st})^{\prime}$.

\section{Lambek's Restriction}

The original Lambek calculus (Lambek, 1958) has an important difference from the systems discussed above, namely Lambek's non-emptiness restriction. Let us start with a linguistic example (Moot and Retoré, 2012, Sect. 2.5). In the calculi defined above, one can derive $(N / N) /(N / N), N \rightarrow N$. This sequent validates "very book" as an object of type $N$ (common noun), which is incorrect. Indeed, the type $(N / N) /(N / N)$ for "very" is a left modifier for adjective, cf. "very interesting book," analyzed as $(N / N) /(N / N), N / N, N \rightarrow N$.

This example motivates the following constraint: left-hand sides of all sequents are required to be non-empty. This constraint existed in the original Lambek calculus (Lambek, 1958). It is quite strange from the logical point of 
view, but is natural from the linguistic side and also in the view of algebraic interpretations (considering residuated semigroups instead of monoids).

In the presence of a full-power exponential modality, however, imposing Lambek's restriction is quite a subtle matter (Kanovich et al., 2016a, 2020). Actually, there is no way of doing it without losing at least one of the desired properties of a good logical system - cut elimination and substitution. Similar issues arise with reconciling Lambek's restriction with the relevant modality.

The subexponential modalities used by Morrill, however, are not that powerful, and their behaviour is constrained by brackets. This makes it possible to impose Lambek's restriction in a linguistically consistent manner. In this section, we present $!_{\mathrm{b}}^{2018} \mathrm{MALCb}(\mathrm{st})^{\prime}$, a version of $!_{\mathrm{b}}^{2018} \mathrm{MALC}{ }^{*} \mathrm{~b}(\mathrm{st})^{\prime}$ with Lambek's restriction imposed.

Before going into the formalism, let us consider one more linguistic example (Morrill, 2018b). This example features an incorrect noun phrase, * "man who likes." The dependent clause here is analysed with two gaps, * "man who [] likes []." The intended semantics (and the correct version of the phrase) is "man who likes himself," that is, both gaps should be filled with the same $N$, using the parasitic extraction mechanism. The lexicon here is the same as in the example in Section 3 .

Since the dependent clause forms a strong (double-bracketed) island, the brackets are imposed as follows: "man [[who likes]]." Next, we recall that the subject should form a weak (single-bracketed) island, and in these brackets can be generated in $!_{\mathrm{b}}^{\mathbf{2 0 1 5}} \mathbf{M A L C} \mathbf{C}^{*} \mathbf{b}(\mathrm{st})$ by the contraction rule. This allows

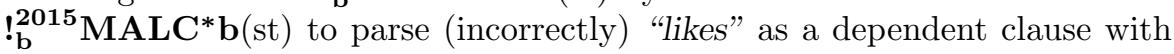
two gaps, a host one for the object and a parasitic one for the subject:

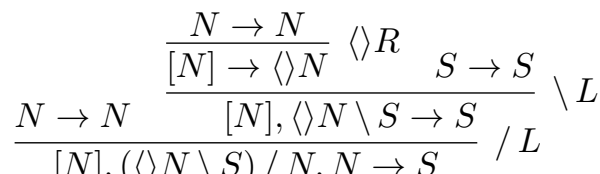

$$
\begin{aligned}
& \frac{\frac{[N],(\langle\rangle N \backslash S) / N, N \rightarrow S}{[N ; \Lambda],(\langle\rangle N \backslash S) / N, N \rightarrow S} \frac{D}{N ;[N ; \Lambda],(\langle>\backslash S) / N \rightarrow S} ! P}{\frac{N ;(\langle\rangle N \backslash S) / N \rightarrow S}{(\langle\rangle N \backslash S) / N, ! N \rightarrow S} ! L} ! C
\end{aligned}
$$

The complete derivation for * "man who likes" as a common noun group $(C N)$ in $!_{\mathbf{b}}^{\mathbf{2 0 1 5}} \mathbf{M A L C} \mathbf{C}^{*} \mathbf{b}(\mathrm{st})$ is given on Figure 3

The problem here is the empty island (subject of the dependent clause) generated by the $! C$ rule. This issue was one of the motivations for Morrill (2018b) to introduce the new system $!_{\mathbf{b}}^{\mathbf{2 0 1 8}} \mathbf{M A L C} \mathbf{B}$ (st), which features another version of ! $C$.

With this new version, the island for parasitic extraction should be given in the bracketing of the goal sequent. Moreover, it should be declared as a strong (double-bracketed) island, and then the $! C$ rule will transform it into a 


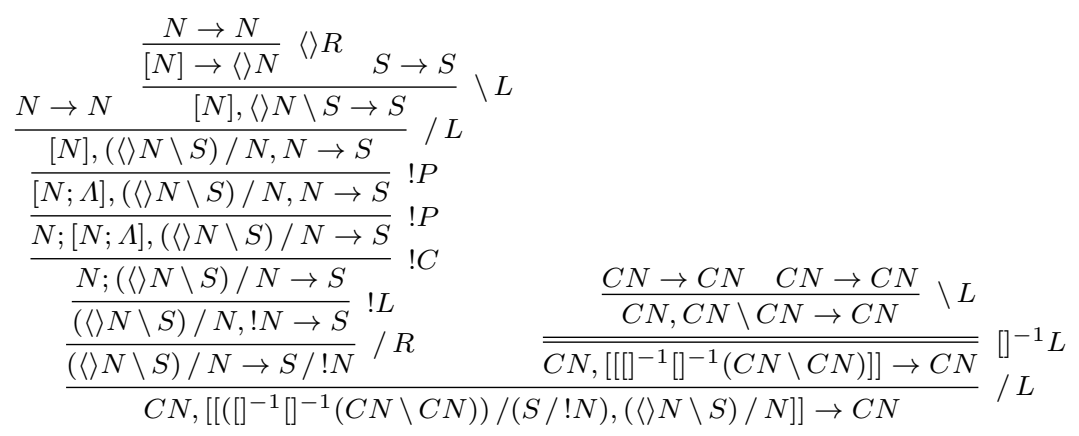

Fig. 3 Derivation for * "man [[who likes]]" in $!_{\mathbf{b}}^{\mathbf{2 0 1 5}} \mathbf{M A L C *} \mathbf{b}(\mathrm{st})$ (cf. Morrill (2018b))

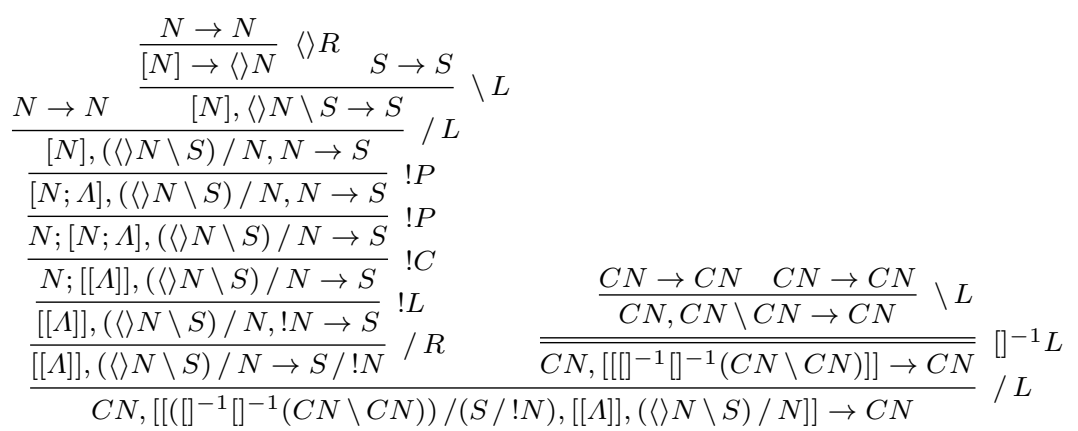

Fig. 4 Derivation of * "man [[who [[ ]] likes]]" in $!_{\mathbf{b}}^{\mathbf{2 0 1 8}} \mathbf{M A L C} \mathbf{C}^{*} \mathbf{b}($ st) (notice the empty subject island)

weak one. The erroneous phrase * "man who likes," however, can still be parsed by $!_{\mathbf{b}}^{\mathbf{2 0 1 8}} \mathbf{M A L C} \mathbf{C}^{*} \mathbf{b}(\mathrm{st})$, but requires the empty subject island to be explicitly introduced in the bracketing, see Figure 4.

An easy way of making phrases like * "man who likes" invalid in grammars based on $!_{\mathbf{b}}^{\mathbf{2 0 1 8}} \mathbf{M A L C}{ }^{*} \mathbf{b}$ (st) is to forbid the user (or an automated bracketinducing system) to put empty bracket domains on the original sentence. This is essentially the idea which motivates the usage of $!_{\mathbf{b}}^{\mathbf{2 0 1 8}} \mathbf{M A L C} \mathbf{C}^{*} \mathbf{b}(\mathrm{st})$ in favour of $!_{b}^{2015} \mathbf{M A L C}{ }^{*} \mathbf{b}(\mathrm{st})$ : in $!_{\mathrm{b}}^{\mathbf{2 0 1 5}} \mathbf{M A L C} \mathbf{C}^{*} \mathbf{b}(\mathrm{st})$, the brackets embracing an empty island appeared only inside the derivation, while in the newer system $!_{\mathbf{b}}^{\mathbf{2 0 1 8}} \mathbf{M A L C}{ }^{*} \mathbf{b}($ st $)$ they should be provided as an input, which could be disallowed externally.

A more logically consistent approach, however, requires imposing nonemptiness restriction systematically for all sequents in derivations. The restriction is formulated as follows:

every meta-formula, both the whole antecedent and each bracketed domain, should be non-empty.

Non-emptiness of a meta-formula means that it is not equal to the empty one, $\varnothing ; \Lambda$. In other words, it should either include a non-empty sequence of formulae, or have a non-empty stoup. 
The first thing one has to do in order to maintain Lambek's restriction is to remove the unit constant $\mathbf{1}$. The unit essentially means "empty," and there is no consistent way of reconciling it with Lambek's restriction. Indeed, having the unit, we can put it into any meta-formula, thus making it formally non-empty. (Unfortunately, it seems that Morrill needs the unit for handling discontinuity, that is why he does not impose Lambek's restriction on his systems.)

Most of the $!_{\mathbf{b}}^{\mathbf{2 0 1 8}} \mathbf{M A L C} \mathbf{C}^{*} \mathbf{b}(\mathrm{st})^{\prime}$ rules keep this restriction, i.e., if in the premises all meta-formulae are non-empty, then the same holds for the conclusion. Only three rules need specifically imposed restrictions:

- for $\backslash R$ and $/ R$, we require that either $\Gamma \neq \Lambda$ or $\zeta \neq \varnothing$ (this is the original Lambek's restriction);

- for the contraction rule, ! $C^{\prime}$, we require that either $\Gamma_{2} \neq \Lambda$ or $\zeta^{\prime} \neq \varnothing$.

The latter constraint exactly captures the idea that parasitic gapping into an empty bracketed island is ungrammatical (cf. the "man that likes" example above).

We denote the version of $\mathbf{~}_{\mathbf{b}}^{\mathbf{2 0 1 8}} \mathbf{M} \mathbf{A L C} \mathbf{C}^{*} \mathbf{b}(\text { st })^{\prime}$ with Lambek's restriction by $!_{\mathrm{b}}^{2018} \mathrm{MALCb}(\mathrm{st})^{\prime}$.

\section{Cut Elimination in Modified Systems}

In this section we prove that the cut rule in the following form

$$
\frac{\xi ; \Pi \rightarrow A \quad \Xi\left(\zeta ; \Gamma_{1}, A, \Gamma_{2}\right) \rightarrow C}{\Xi\left(\xi, \zeta ; \Gamma_{1}, \Pi, \Gamma_{2}\right) \rightarrow C} \mathrm{cut}
$$

is admissible in the following calculi: $!_{\mathbf{b}}^{\mathbf{2 0 1 8}} \mathbf{M A L C} \mathbf{A}(\mathrm{st})^{\prime}, !_{\mathbf{b}}^{\mathbf{2 0 1 8}} \mathbf{M A L C b}(\mathrm{st})^{\prime}$, and $!_{\mathrm{b}}^{\mathbf{2 0 1 5}} \mathbf{M A L C} \mathbf{C}^{*} \mathbf{b}(\mathrm{st})^{\prime}$. We show this by a single inductive argument for $!_{\mathrm{b}}^{2018} \mathrm{MALC}{ }^{*} \mathrm{~b}(\mathrm{st})^{\prime}$ and $!_{\mathrm{b}}^{\mathbf{2 0 1 8}} \mathrm{MALCb}(\mathrm{st})^{\prime}$, and then make necessary changes for $!_{\mathrm{b}}^{2015} \mathrm{MALC}{ }^{*} \mathrm{~b}(\mathrm{st})^{\prime}$.

Theorem 1 Let sequents $\xi ; \Pi \rightarrow A$ and $\Xi\left(\zeta ; \Gamma_{1}, A, \Gamma_{2}\right) \rightarrow C$ be derivable in $!_{\mathbf{b}}^{\mathbf{2 0 1 8}} \mathbf{M A L C}{ }^{*} \mathbf{b}(\mathrm{st})^{\prime}$ or $!_{\mathbf{b}}^{\mathbf{2 0 1 8}} \mathbf{M A L C b}(\mathrm{st})^{\prime}$. Then $\Xi\left(\xi, \zeta ; \Gamma_{1}, \Pi, \Gamma_{2}\right) \rightarrow C$ is also derivable in $!_{\mathbf{b}}^{\mathbf{2 0 1 8}} \mathbf{M A L C} \mathbf{M}(\mathrm{st})^{\prime}$ or, respectively, $!_{\mathbf{b}}^{\mathbf{2 0 1 8}} \mathbf{M A L C b}(\mathrm{st})^{\prime}$.

The proof of cut elimination traditionally goes by nested induction: on the complexity of the formula being cut, and on the depth of the cut, that is, the number of rules applied in the derivation above the cut.

For the original Lambek calculus, cut elimination was shown by Lambek (1958). Moortgat (1996) extended Lambek's proof to the Lambek calculus with brackets. The presence of ! and stoups, however, makes cut elimination more involved. Namely, the principal case for ! moves the active formula being cut to the stoup:

$$
\frac{\frac{B ; \Lambda \rightarrow A}{B ; \Lambda \rightarrow ! A} ! R^{\prime} \quad \frac{\Xi\left(\zeta, A ; \Gamma_{1}, \Gamma_{2}\right) \rightarrow C}{\Xi\left(\zeta ; \Gamma_{1}, ! A, \Gamma_{2}\right) \rightarrow C} ! L}{\Xi\left(\zeta, B ; \Gamma_{1}, \Gamma_{2}\right) \rightarrow C} \mathrm{cut}
$$


Propagating the cut upwards in this situation would require a specific version of cut for formulae inside the stoup, and eliminate it together with the usual cut rule by simultaneous induction. Contraction, however, raises yet another issue with propagating cut. Namely, if we contract the formula $A$ being cut, then after propagation we get two cut applications, one under another. For the lower cut, we fail to maintain the decrease of induction parameters, see Kanovich et al. (2019a).

The standard strategy, going back to Gentzen (1935) and applied to linear logic with exponentials by Girard (1987) and Lincoln et al. (1992), replaces the cut rule with a more general rule called mix. Mix is a combination of cut and contractions, and this more general rule is then eliminated by a straightforward inductive argument. In the presence of brackets and stoups, however, formulating mix becomes an extremely tedious job. In the view of that, we follow another strategy, "deep cut elimination" by Braüner and de Paiva (1996. 1998); see also Braüner (2000); Eades III and de Paiva (2020).

Proof Let $\xi ; \Pi \rightarrow A$ and $\Xi\left(\zeta ; \Gamma_{1}, A, \Gamma_{2} \rightarrow C\right)$ have cut-free derivations $\mathscr{D}_{\text {left }}$ and $\mathscr{D}_{\text {right }}$ respectively. We proceed by nested induction on two parameters: $\kappa$, the complexity of the formula $A$ being cut; $\sigma$, the total number of rule applications in the derivations of $\mathscr{D}_{\text {left }}$ and $\mathscr{D}_{\text {right }}$. In each case either $\kappa$ gets reduces, or $\sigma$ gets reduced with the same $\kappa$.

We consider the lowermost rules of $\mathscr{D}_{\text {left }}$ and $\mathscr{D}_{\text {right }}$.

We call !P and !C structural rules; all other rules (excluding cut, which is not allowed in our derivations) are logical ones. Being the lowermost rule of $\mathscr{D}_{\text {left }}$ or $\mathscr{D}_{\text {right }}$, a logical rule is called principal, if it introduces the formula $A$ being cut. The axiom $\Lambda \rightarrow \mathbf{1}$ in this proof is considered a principal rule (with no premises) introducing 1 . Structural rules are never principal, since they operate only inside the stoup, while the formula $A$ being cut is not in the stoup.

First we list all possible cases, with short comments, and then accurately consider each of them:

1. The lowermost rule in $\mathscr{D}_{\text {left }}$ is $! R$ and the lowermost rule in $\mathscr{D}_{\text {right }}$ is $! L$ (i.e., the principal case with !). This is actually the most interesting case, in which deep cut elimination differs from traditional cut elimination schemes. In this case, we are going to perform a non-local transformation of the $\mathscr{D}_{\text {right }}$ tree, as shown below.

2. Both lowermost rules of $\mathscr{D}_{\text {left }}$ and $\mathscr{D}_{\text {right }}$ are principal, and $A$ is not of the form $! A^{\prime}$ (if it is, we are in Case 1 ). This is the standard principal case for cut elimination in the Lambek calculus: the tricky part with ! is considered in Case 1, not here.

3. The lowermost rule in $\mathscr{D}_{\text {left }}$ is a non-principal one. In this case we propagate cut to the left.

4. The lowermost rule in $\mathscr{D}_{\text {right }}$ is a non-principal one. Propagate cut to the right.

5. One of the premises of cut is an axiom of the form $A \rightarrow A$. Cut disappears. 


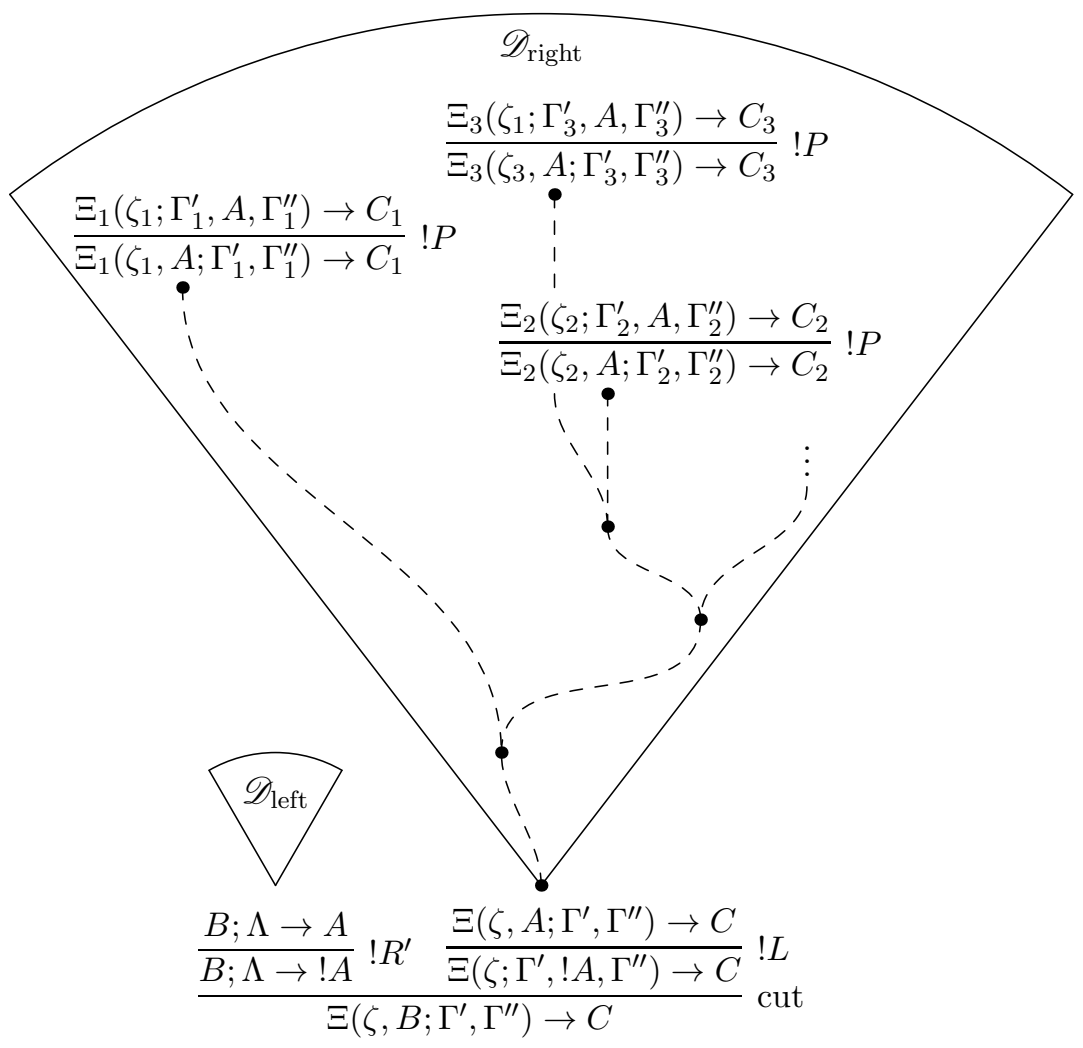

Fig. 5 Tracing $A$ in the stoup up to $! P$

Case 1 (deep: principal for !): the lowermost rule in $\mathscr{D}_{\text {left }}$ is $! R$ and the lowermost rule in $\mathscr{D}_{\text {right }}$ is $! L$. Cut is applied as follows:

$$
\frac{\frac{B ; \Lambda \rightarrow A}{B ; \Lambda \rightarrow ! A} ! R \quad \frac{\left.\Xi\left(\zeta, A ; \Gamma^{\prime}, \Gamma^{\prime \prime}\right) \rightarrow C\right)}{\Xi\left(\zeta ; \Gamma^{\prime}, ! A, \Gamma^{\prime \prime}\right) \rightarrow C} ! L}{\Xi\left(\zeta, B ; \Gamma^{\prime}, \Gamma^{\prime \prime}\right) \rightarrow C} \mathrm{cut}
$$

Let us trace the designated occurrence of $A$ inside the stoup upwards along $\mathscr{D}_{\text {right }}$. Each principal ! $C^{\prime}$ application branches the trace. The trace also branches on applications of $\wedge R$ and $\vee L$. Each branch ends at a principal application of $! P$ (see Figure 5).

Now we perform the deep cut elimination step. In $\mathscr{D}_{\text {right }}$, we replace the designated occurrences of $A$ in the stoup with $B$. The applications of $! C^{\prime}$ remain valid. Other rules do not operate $A$ in the stoup and therefore remain intact. After this replacement applications of $! P$ transform into applications of cut with $B ; \Lambda \rightarrow A$ as the left premise (Figure 6). One trace could go through several instances of $! P$ with the active $A$, like $\Xi_{2}$ and $\Xi_{3}$ in the example; in this case we go from top to bottom. 


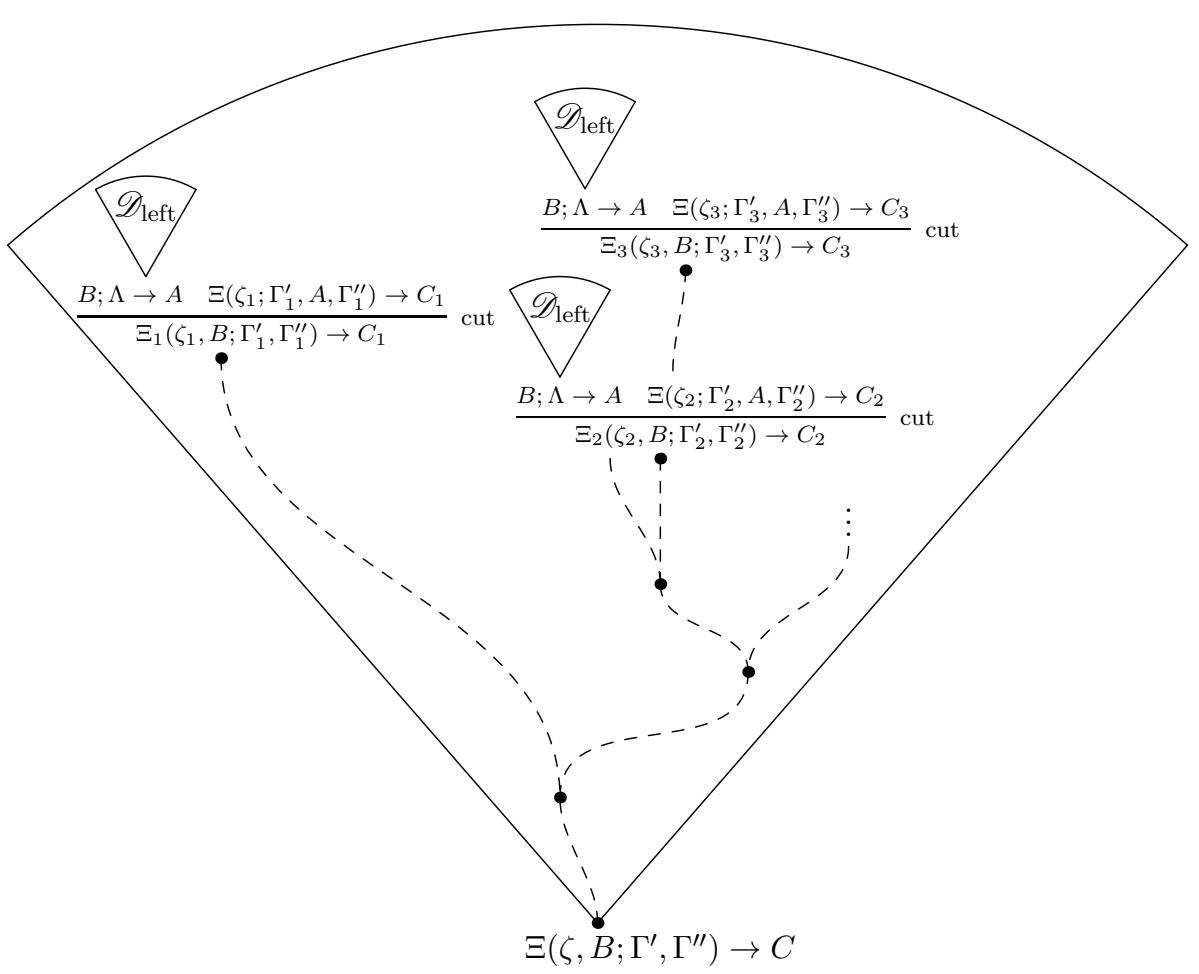

Fig. 6 Deep cut elimination

The new cuts have lower $\kappa$ (the cut formula is $A$ instead of $! A)$, and therefore they are eliminable by induction hypothesis.

For the case with Lambek's restriction, notice that in the deep cut elimination step we just changed $A$ to $B$ in the stoups, so Lambek's restriction could not get violated.

The figures illustrating deep cut elimination (Figure 5 and Figure 60 are taken from Kanovich et al. (2017b), with the necessary changes for calculi with stoups.

Case 2 (principal, but not !). As said before, this case is the standard principal case for cut elimination in the multiplicative-additive Lambek calculus with brackets (Moortgat, 1996), since ! does not appear in this case. Adding the stoups makes only a minor difference. All the interesting things about ! have already happened in the "deep" Case 1.

So, the main connective of $A$ is not !. Consider other possible cases.

Subcase 2.a. $A=A_{1} \backslash A_{2}$ or $A=A_{2} / A_{1}$ (the latter is of course symmetric to the former). In this case

$$
\frac{\frac{\xi ; A_{1}, \Pi \rightarrow A_{2}}{\xi ; \Pi \rightarrow A_{1} \backslash A_{2}} \backslash R \quad \frac{\zeta_{1} ; \Gamma \rightarrow A_{1} \quad \Xi\left(\zeta_{2} ; \Delta_{1}, A_{2}, \Delta_{2}\right) \rightarrow C}{\Xi\left(\zeta_{1}, \zeta_{2} ; \Delta_{1}, \Gamma, A_{1} \backslash A_{2}, \Delta_{2}\right) \rightarrow C} \backslash L}{\Xi\left(\xi, \zeta_{1}, \zeta_{2} ; \Delta_{1}, \Gamma, \Pi, \Delta_{2}\right) \rightarrow C} \text { cut }
$$


transforms into

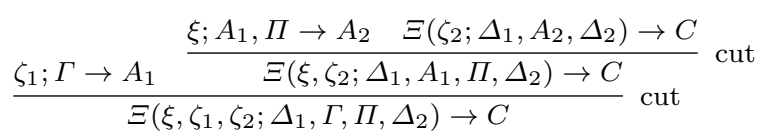

Both new cuts have a smaller $\kappa$ parameter (and then we do not care for $\sigma$ ).

Here in the new derivation no new $\backslash R$ instance was added, so Lambek's restriction is observed.

Subcase 2.b. $A=A_{1} \cdot A_{2}$. In this case

$$
\frac{\frac{\xi_{1} ; \Pi_{1} \rightarrow A_{1} \quad \xi_{2} ; \Pi_{2} \rightarrow A_{2}}{\xi_{1}, \xi_{2} ; \Pi_{1}, \Pi_{2} \rightarrow A_{1} \cdot A_{2}} \cdot R \frac{\Xi\left(\zeta ; \Delta_{1}, A_{1}, A_{2}, \Delta_{2}\right) \rightarrow C}{\Xi\left(\zeta ; \Delta_{1}, A_{1} \cdot A_{2}, \Delta_{2}\right) \rightarrow C}}{\Xi\left(\xi_{1}, \xi_{2}, \zeta ; \Delta_{1}, \Pi_{1}, \Pi_{2}, \Delta_{2}\right) \rightarrow C} \cdot L
$$

transforms into

$$
\frac{\xi_{1} ; \Pi_{1} \rightarrow A_{1} \frac{\xi_{2} ; \Pi_{2} \rightarrow A_{2} \quad \Xi\left(\zeta ; \Delta_{1}, A_{1}, A_{2}, \Delta_{2}\right) \rightarrow C}{\Xi\left(\xi_{2}, \zeta ; \Delta_{1}, A_{1}, \Pi_{2}, \Delta_{2}\right) \rightarrow C}}{\Xi\left(\xi_{1}, \xi_{2}, \zeta ; \Delta_{1}, \Pi_{1}, \Pi_{2}, \Delta_{2}\right) \rightarrow C} \text { cut } \mathrm{cut}
$$

(again $\kappa$ decreases).

Subcase 2.c. $A=\mathbf{1}$ :

$$
\frac{\Lambda \rightarrow \mathbf{1} \frac{\Xi\left(\zeta ; \Delta_{1}, \Delta_{2}\right) \rightarrow C}{\Xi\left(\zeta ; \Delta_{1}, \mathbf{1}, \Delta_{2}\right) \rightarrow C}}{\Xi\left(\zeta ; \Delta_{1}, \Delta_{2}\right) \rightarrow C} \text { cut }
$$

The goal coincides with the right premise, so we just remove this detour and arrive at a cut-free proof of $\Xi\left(\zeta ; \Delta_{1}, \Delta_{2}\right) \rightarrow C$.

Subcase 2.d. $A=A_{1} \vee A_{2}$ :

$$
\frac{\frac{\xi ; \Pi \rightarrow A_{i}}{\xi ; \Pi \rightarrow A_{1} \vee A_{2}} \vee R_{i} \frac{\Xi\left(\zeta ; \Delta_{1}, A_{1}, \Delta_{2}\right) \rightarrow C \quad \Xi\left(\zeta ; \Delta_{1}, A_{2}, \Delta_{2}\right) \rightarrow C}{\Xi\left(\zeta ; \Delta_{1}, A_{1} \vee A_{2}, \Delta_{2}\right) \rightarrow C}}{\Xi\left(\xi, \zeta ; \Delta_{1}, \Pi, \Delta_{2}\right) \rightarrow C} \vee L
$$

transforms into

$$
\frac{\xi ; \Pi \rightarrow A_{i} \quad \Xi\left(\zeta ; \Delta_{1}, A_{i}, \Delta_{2}\right) \rightarrow C}{\Xi\left(\xi, \zeta ; \Delta_{1}, \Pi, \Delta_{2}\right) \rightarrow C} \mathrm{cut}
$$

( $\kappa$ gets decreased, and the derivation of $\Pi \rightarrow A_{j}$ for $j \neq i$ gets forgotten).

Subcase 2.e. $A=A_{1} \wedge A_{2}$ :

$$
\frac{\frac{\xi ; \Pi \rightarrow A_{1} \quad \xi ; \Pi \rightarrow A_{2}}{\xi ; \Pi \rightarrow A_{1} \wedge A_{2}} \wedge R \quad \frac{\Xi\left(\xi, \zeta ; \Delta_{1}, A_{j}, \Delta_{2}\right) \rightarrow C}{\Xi\left(\xi, \zeta ; \Delta_{1}, A_{1} \wedge A_{2}, \Delta_{2}\right) \rightarrow C}}{\Xi\left(\xi, \zeta ; \Delta_{1}, \Pi, \Delta_{2}\right) \rightarrow C} \text { cut }
$$

transforms into

$$
\frac{\xi ; \Pi \rightarrow A_{j} \quad \Xi\left(\xi, \zeta ; \Delta_{1}, A_{j}, \Delta_{2}\right) \rightarrow C}{\Xi\left(\xi, \zeta ; \Delta_{1}, \Pi, \Delta_{2}\right) \rightarrow C} \mathrm{cut}
$$

( $\kappa$ gets decreased, and the derivation of $\xi ; \Pi \rightarrow A_{i}$ for $i \neq j$ gets forgotten). 
Subcase 2.f. $A=\langle\rangle A^{\prime}$. Notice that here $\xi$ is empty, otherwise \langle\rangle $R$ could not be applied.

$$
\frac{\frac{\Pi \rightarrow A^{\prime}}{[\Pi] \rightarrow\langle\rangle A^{\prime}}\langle\rangle R \frac{\Xi\left(\zeta ; \Delta_{1},\left[A^{\prime}\right], \Delta_{2}\right) \rightarrow C}{\Xi\left(\zeta ; \Delta_{1},\langle\rangle A^{\prime}, \Delta_{2} \rightarrow C\right.}}{\Xi\left(\zeta ; \Delta_{1},[\Pi], \Delta_{2}\right) \rightarrow C} \text { cut }
$$

transforms into

$$
\frac{\Pi \rightarrow A^{\prime} \quad \Xi\left(\zeta ; \Delta_{1},\left[A^{\prime}\right], \Delta_{2}\right) \rightarrow C}{\Xi\left(\zeta ; \Delta_{1},[\Pi], \Delta_{2}\right) \rightarrow C} \mathrm{cut}
$$

( $\kappa$ decreases).

Subcase 2.g. $A=[]^{-1} A^{\prime}$. In this subcase, notice that the stoup of the metaformula including the active []$^{-1} A^{\prime}$ is empty, by the []$^{-1} L$ rule; $\zeta^{\prime}$ below is the stoup of a different bracketed domain.

$$
\left.\frac{\frac{[\xi ; \Pi] \rightarrow A^{\prime}}{\xi ; \Pi \rightarrow[]^{-1} A^{\prime}}[]^{-1} R \quad \frac{\Xi\left(\zeta^{\prime} ; \Delta_{1}, A^{\prime}, \Delta_{2}\right) \rightarrow C}{\Xi\left(\zeta^{\prime} ; \Delta_{1},\left[[]^{-1} A^{\prime}\right], \Delta_{2}\right) \rightarrow C}}{\Xi\left(\zeta^{\prime} ; \Delta_{1},[\xi ; \Pi], \Delta_{2}\right) \rightarrow C} \text { cut }\right]^{-1} L
$$

transforms into

$$
\frac{[\xi ; \Pi] \rightarrow A^{\prime} \quad \Xi\left(\zeta^{\prime} ; \Delta_{1}, A^{\prime}, \Delta_{2}\right) \rightarrow C}{\Xi\left(\zeta^{\prime} ; \Delta_{1},[\xi ; \Pi], \Delta_{2}\right) \rightarrow C} \text { cut }
$$

$(\kappa$ decreases).

Case 3 (left non-principal). The lowermost rule of $\mathscr{D}_{\text {left }}$ is non-principal if and only if it is a left rule, i.e., operates in the antecedent.

Subcase 3.a. The lowermost rule in $\mathscr{D}_{\text {left }}$ is one of the one-premise rules, $\cdot L$, $1 L, \vee L,\langle\rangle L,[]^{-1} L, ! L, ! P, ! C^{\prime}$. Such rules are called easy rules in Kanovich et al. (2019a), therefore we denote this rule by $E R$ :

$$
\frac{\frac{\xi^{\prime} ; \Pi^{\prime} \rightarrow A}{\xi ; \Pi \rightarrow A} E R \quad \Xi\left(\zeta ; \Gamma_{1}, A, \Gamma_{2}\right) \rightarrow C}{\Xi\left(\xi, \zeta ; \Gamma_{1}, \Pi, \Gamma_{2}\right) \rightarrow C} \mathrm{cut}
$$

The easy rule is still valid in a larger context, where $\Pi$ is put between $\Gamma_{1}$ and $\Gamma_{2}$ and $\xi$ is added to $\zeta$, therefore one can reconstruct the derivation as follows:

$$
\frac{\xi^{\prime} ; \Pi^{\prime} \rightarrow A \quad \Xi\left(\zeta ; \Gamma_{1}, A, \Gamma_{2}\right) \rightarrow C}{\frac{\Xi\left(\xi^{\prime}, \zeta ; \Gamma_{1}, \Pi^{\prime}, \Gamma_{2}\right) \rightarrow C}{\Xi\left(\xi, \zeta ; \Gamma_{1}, \Pi, \Gamma_{2}\right) \rightarrow C} E R} \text { cut }
$$

The new cut has a smaller $\sigma$ with the same $\kappa$, and can be eliminated by induction. Lambek's restriction on! $C^{\prime}$, if it was imposed, is kept.

Subcase 3.b. The lowermost rule in $\mathscr{D}_{\text {left }}$ is $\backslash L$ or $/ L$. Here

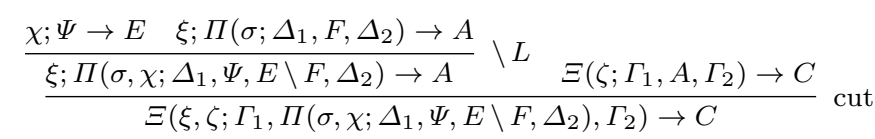

transforms into

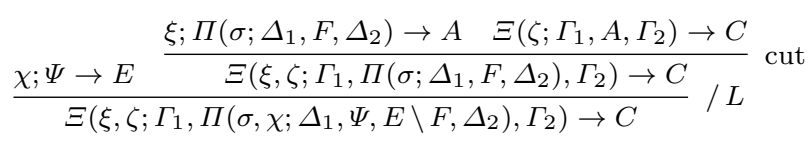


In particular, $\Pi$ could just coincide with the designated meta-formula inside. In this case $\sigma, \chi$ gets merged with $\xi, \zeta$ into one stoup.

The new cut has a smaller $\sigma$ with the same $\kappa$. The / case is symmetric.

Subcase 3.c. The lowermost rule in $\mathscr{D}_{\text {left }}$ is $\vee L$. Then a derivation of the sequent $\Xi\left(\xi, \zeta ; \Gamma_{1}, \Pi\left(\sigma ; \Delta_{1}, B_{1} \vee B_{2}, \Delta_{2}\right), \Gamma_{2}\right) \rightarrow C$ by cut from $\xi ; \Pi\left(\sigma ; \Delta_{1}, B_{1} \vee\right.$ $\left.B_{2}, \Delta_{2}\right) \rightarrow A$ and $\Xi\left(\zeta ; \Gamma_{1}, A, \Gamma_{2}\right) \rightarrow C$ is transformed into a derivation by $\vee L$, whose premises are derived as follows $(i=1,2)$ :

$$
\frac{\xi ; \Pi\left(\sigma ; \Delta_{1}, B_{i}, \Delta_{2}\right) \rightarrow A \quad \Xi\left(\zeta ; \Gamma_{1}, A, \Gamma_{2}\right) \rightarrow C}{\Xi\left(\xi, \zeta ; \Gamma_{1}, \Pi\left(\sigma ; \Delta_{1}, B_{i}, \Delta_{2}\right), \Gamma_{2}\right) \rightarrow C} \mathrm{cut}
$$

In particular, $\Pi$ could just coincide with the designated meta-formula inside. In this case $\sigma$ gets merged with $\xi, \zeta$ into one stoup.

Now we have two cuts, but each of it has a smaller $\sigma$ parameter with the same $\kappa$, and they are independent, i.e., derivations of the premises of these cuts are already cut-free. Thus, we proceed by induction.

\section{Case 4 (right non-principal).}

Subcase 4.a. In the right case we also have the notion of easy rule, which is a one-premise rule that does something in the context $\left(\Xi, \zeta, \Gamma_{1}, \Gamma_{2}, C\right)$ while keeping the active occurrence of $A$ intact. These rules are non-principal instances of $\backslash R, / R, \cdot L, \mathbf{1} L, \wedge L_{i}, \vee R_{i},\langle\rangle L,\langle\rangle R,[]^{-1} L,[]^{-1} R, ! L, ! P$. Contraction, ! $C^{\prime}$, is also essentially an easy rule, but we consider it more accurately below (Subcase $3 \cdot \mathrm{a}^{\prime}$ ). For easy rules, the transformation is as follows:

$$
\frac{\xi ; \Pi \rightarrow A \frac{\Xi^{\prime}\left(\zeta^{\prime} ; \Gamma_{1}^{\prime}, A, \Gamma_{2}^{\prime}\right) \rightarrow C^{\prime}}{\Xi\left(\zeta ; \Gamma_{1}, A, \Gamma_{2}\right) \rightarrow C}}{\Xi\left(\xi, \zeta ; \Gamma_{1}, \Pi, \Gamma_{2}\right) \rightarrow C} \mathrm{cut}
$$

transforms into

$$
\frac{\xi ; \Pi \rightarrow A \quad \Xi^{\prime}\left(\zeta^{\prime} ; \Gamma_{1}^{\prime}, A, \Gamma_{2}^{\prime}\right) \rightarrow C^{\prime}}{\frac{\Xi^{\prime}\left(\xi, \zeta^{\prime} ; \Gamma_{1}^{\prime}, \Pi, \Gamma_{2}^{\prime}\right) \rightarrow C^{\prime}}{\Xi\left(\xi, \zeta ; \Gamma_{1}, \Pi, \Gamma_{2}\right) \rightarrow C} E R} \text { cut }
$$

which is legal, since the easy rule is still valid with $\Pi$ substituted for $A$ (recall that $A$ was not the active formula in the easy rule) and $\xi$ added to the stoup. Moreover, if the original application of the easy rule, $\backslash R$ or $/ R$, obeyed Lambek's restriction, so will the new one. The $\sigma$ parameter decreases, with the same $\kappa$.

Subcase 4.a'. The lowermost rule in $\mathscr{D}_{\text {right }}$ is $! C^{\prime}$. The interesting situation here is when contraction and cut are performed in the same meta-formula; otherwise ! $C^{\prime}$ acts as an easy rule, considered in the previous subcase. Since $! C^{\prime}$ operates two bracketed domains (the outer domain and the island), we have two subsituations.

Namely,

$$
\frac{\xi ; \Pi \rightarrow A}{\Xi\left(\xi, \zeta, B ; \Gamma_{1}^{\prime}, \Pi, \Gamma_{1}^{\prime \prime},\left[\left[\zeta_{1}^{\prime} ; \Gamma_{2}\right]\right], \Gamma_{3}\right) \rightarrow C} \mathrm{\Xi ut}
$$


transforms into

$$
\frac{\xi ; \Pi \rightarrow A \quad \Xi\left(\zeta, B ; \Gamma_{1}^{\prime}, A, \Gamma_{1}^{\prime \prime},\left[\zeta^{\prime}, B ; \Gamma_{2}\right], \Gamma_{3}\right) \rightarrow C}{\frac{\Xi\left(\xi, \zeta, B ; \Gamma_{1}^{\prime}, \Pi, \Gamma_{1}^{\prime \prime},\left[\zeta^{\prime}, B ; \Gamma_{2}\right], \Gamma_{3}\right) \rightarrow C}{\Xi\left(\xi, \zeta, B ; \Gamma_{1}^{\prime}, \Pi, \Gamma_{1}^{\prime \prime},\left[\left[\zeta^{\prime} ; \Gamma_{2}\right]\right], \Gamma_{3}\right) \rightarrow C} ! C^{\prime}} \mathrm{cut}
$$

(the case with $A$ in $\Gamma_{3}$ is considered symmetrically), and

$$
\frac{\xi ; \Pi \rightarrow A \frac{\Xi\left(\zeta, B ; \Gamma_{1},\left[\zeta^{\prime}, B ; \Gamma_{2}^{\prime}, A, \Gamma_{2}^{\prime \prime}\right], \Gamma_{3}\right) \rightarrow C}{\Xi\left(\zeta, B ; \Gamma_{1},\left[\left[\zeta^{\prime} ; \Gamma_{2}^{\prime}, A, \Gamma_{2}^{\prime \prime}\right]\right], \Gamma_{3}\right) \rightarrow C} ! C^{\prime}}{\Xi\left(\zeta, B ; \Gamma_{1},\left[\left[\xi, \zeta^{\prime} ; \Gamma_{2}^{\prime}, \Pi, \Gamma_{2}^{\prime \prime}\right]\right], \Gamma_{3}\right) \rightarrow C} \text { cut }
$$

transforms into

$$
\frac{\xi ; \Pi \rightarrow A \quad \Xi\left(\zeta, B ; \Gamma_{1},\left[\zeta^{\prime}, B ; \Gamma_{2}^{\prime}, A, \Gamma_{2}^{\prime \prime}\right], \Gamma_{3}\right) \rightarrow C}{\frac{\Xi\left(\zeta, B ; \Gamma_{1},\left[\xi, \zeta^{\prime}, B ; \Gamma_{2}^{\prime}, \Pi, \Gamma_{2}^{\prime \prime}\right], \Gamma_{3}\right) \rightarrow C}{\Xi\left(\zeta, B ; \Gamma_{1},\left[\left[\xi, \zeta^{\prime} ; \Gamma_{2}^{\prime}, \Pi, \Gamma_{2}^{\prime \prime}\right]\right], \Gamma_{3}\right) \rightarrow C} ! C^{\prime}} \mathrm{cut}
$$

The $\sigma$ parameter decreases, with the same $\kappa$. Lambek's restriction on $! C$, if imposed, is kept.

Subcase $4 . b$ (the counterpart of $3 . \mathrm{b}$ ). The lowermost rule in $\mathscr{D}_{\text {right }}$ is a nonprincipal instance of $\backslash L$ or $/ L$. We consider $\backslash L ; / L$ is symmetric. There are three possible situations, depending on the relative locations of the active $A$ of cut and the active $E \backslash F$ of $\backslash L$.

If the active $A$ goes to the left premise of $\backslash L$, then

$$
\frac{\xi ; \Pi \rightarrow A \quad \frac{\chi ; \Phi\left(\zeta ; \Gamma_{1}, A, \Gamma_{2}\right) \rightarrow E \quad \Xi\left(\sigma ; \Delta_{1}, F, \Delta_{2}\right) \rightarrow C}{\Xi\left(\chi, \sigma ; \Delta_{1}, \Phi\left(\zeta ; \Gamma_{1}, A, \Gamma_{2}\right), E \backslash F, \Delta_{2}\right) \rightarrow C}}{\Xi\left(\chi, \sigma ; \Delta_{1}, \Phi\left(\xi, \zeta ; \Gamma_{1}, \Pi, \Gamma_{2}\right), E \backslash F, \Delta_{2}\right) \rightarrow C} \text { cut }
$$

transforms into

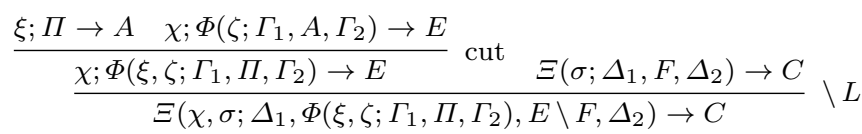

In particular, $\Phi$ could be just $\xi, \zeta ; \Gamma_{1}, \Pi, \Gamma_{2}$, in which case $\xi, \zeta$ gets merged with $\chi, \sigma$ into one stoup.

If the active $A$ goes to the right premise of $\backslash L$, there are two more cases:

$$
\frac{\xi ; \Pi \rightarrow A \quad \frac{\chi ; \Phi \rightarrow E \quad \Xi\left(\sigma ; \Delta_{1}\left(\zeta ; \Gamma_{1}, A, \Gamma_{2}\right), F, \Delta_{2}\right) \rightarrow C}{\Xi\left(\chi, \sigma ; \Delta_{1}\left(\zeta ; \Gamma_{1}, A, \Gamma_{2}\right), \Phi, E \backslash F, \Delta_{2}\right) \rightarrow C}}{\Xi\left(\chi, \sigma ; \Delta_{1}\left(\xi, \zeta ; \Gamma_{1}, \Pi, \Gamma_{2}\right), \Phi, E \backslash F, \Delta_{2}\right) \rightarrow C} \text { cut }
$$

transforms into

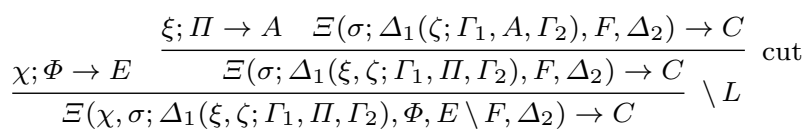


(in particular, $\Delta_{1}$ could be just $\xi, \zeta ; \Gamma_{1}, \Pi, \Gamma_{2}$, in which case $\xi, \zeta$ gets merged with $\chi, \sigma$ into one stoup; the $\Delta_{2}$ case is symmetric), and, finally,

$$
\frac{\xi ; \Pi \rightarrow A \quad \frac{\chi ; \Phi \rightarrow E \quad \Xi\left(\zeta ; \Gamma_{1}, A, \Gamma_{2}\right)\left(\sigma ; \Delta_{1}, F, \Delta_{2}\right) \rightarrow C}{\Xi\left(\zeta ; \Gamma_{1}, A, \Gamma_{2}\right)\left(\chi, \sigma ; \Delta_{1}, \Phi, E \backslash F, \Delta_{2}\right) \rightarrow C} \backslash L}{\Xi\left(\xi, \zeta ; \Gamma_{1}, \Pi, \Gamma_{2}\right)\left(\chi, \sigma ; \Delta_{1}, \Phi, E \backslash F, \Delta_{2}\right) \rightarrow C} \text { cut }
$$

transforms into

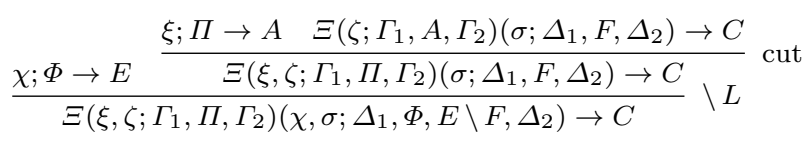

The notation $\Xi(\ldots)(\ldots)$ means a meta-formula with two designated sub-metaformulae, which are independent (i.e., do not intersect).

The $\sigma$ parameter decreases, with the same $\kappa$. $\cdot R$

Subcase 4.c (similar to the previous one). The lowermost rule of $\mathscr{D}_{\text {right }}$ is

$$
\frac{\xi ; \Pi \rightarrow A}{\sigma_{1}, \sigma_{2} ; \Gamma_{1}\left(\xi, \zeta ; \Delta_{1}, \Pi, \Delta_{2}\right), \Gamma_{2} \rightarrow E \cdot F} \mathrm{cut}
$$

transforms into

$$
\frac{\xi ; \Pi \rightarrow A \quad \sigma_{1} ; \Gamma_{1}\left(\zeta ; \Delta_{1}, A, \Delta_{2}\right) \rightarrow E}{\frac{\sigma_{1} ; \Gamma_{1}\left(\xi, \zeta ; \Delta_{1}, \Pi, \Delta_{2}\right) \rightarrow E}{\sigma_{1}, \sigma_{2} ; \Gamma_{1}\left(\xi, \zeta ; \Delta_{1}, \Pi, \Delta_{2}\right), \Gamma_{2} \rightarrow E \cdot F} \sigma_{2} ; \Gamma_{2} \rightarrow F} \cdot R
$$

Again, $\Gamma_{1}$ could coincide with its designated part; in this situation, $\xi, \zeta$ gets merged into $\sigma_{1}$. The case where $A$ appears in $\Gamma_{2}$ is symmetric.

Here $\sigma$ decreases, with the same $\kappa$.

Subcase 4.d (the counterpart of 3.c). The lowermost rule in $\mathscr{D}_{\text {right }}$ is $\vee L$. Here we have two cases, depending on the mutual location of the active $A$ of cut and the active $B_{1} \vee B_{2}$ of $\vee L$. Namely, if they are in different (nonintersecting) meta-formulae, then

$$
\frac{\xi ; \Pi \rightarrow A \frac{\Xi\left(\zeta ; \Gamma_{1}, A, \Gamma_{2}\right)\left(\sigma ; \Delta_{1}, B_{1}, \Delta_{2}\right) \rightarrow C \quad \Xi\left(\zeta ; \Gamma_{1}, A, \Gamma_{2}\right)\left(\sigma ; \Delta_{1}, B_{2}, \Delta_{2}\right) \rightarrow C}{\Xi\left(\zeta ; \Gamma_{1}, A, \Gamma_{2}\right)\left(\sigma ; \Delta_{1}, B_{1} \vee B_{2}, \Delta_{2}\right) \rightarrow C} \mathrm{cut}}{\Xi\left(\xi, \zeta ; \Gamma_{1}, \Pi, \Gamma_{2}\right)\left(\sigma ; \Delta_{1}, B_{1} \vee B_{2}, \Delta_{2}\right) \rightarrow C} \vee L
$$

transforms into two cuts $(i=1,2)$ with simpler cut formulae:

$$
\frac{\xi ; \Pi \rightarrow A \quad \Xi\left(\zeta ; \Gamma_{1}, A, \Gamma_{2}\right)\left(\sigma ; \Delta_{1}, B_{i}, \Delta_{2}\right) \rightarrow C}{\Xi\left(\xi, \zeta ; \Gamma_{1}, \Pi, \Gamma_{2}\right)\left(\sigma ; \Delta_{1}, B_{i}, \Delta_{2}\right) \rightarrow C} \text { cut }
$$

whose goals are then merged by $\vee L$. and if $B_{1} \vee B_{2}$ is in $\Gamma_{1}$ (or, symmetrically, in $\Gamma_{2}$ ), then

$$
\frac{\xi ; \Pi \rightarrow A \frac{\Xi\left(\zeta ; \Gamma_{1}\left(\sigma ; \Delta_{1}, B_{1}, \Delta_{2}\right), A, \Gamma_{2}\right) \rightarrow C \quad \Xi\left(\zeta ; \Gamma_{1}\left(\sigma ; \Delta_{1}, B_{2}, \Delta_{2}\right), A, \Gamma_{2}\right) \rightarrow C}{\Xi\left(\zeta ; \Gamma_{1}\left(\sigma ; \Delta_{1}, B_{1} \vee B_{2}, \Delta_{2}\right), A, \Gamma_{2}\right) \rightarrow C}}{\Xi\left(\xi, \zeta ; \Gamma_{1}\left(\sigma ; \Delta_{1}, B_{1} \vee B_{2}, \Delta_{2}\right), \Pi, \Gamma_{2}\right) \rightarrow C} \vee L
$$


transforms into two cuts $(i=1,2)$, whose goals again can be merged by $\vee L$ :

$$
\frac{\xi ; \Pi \rightarrow A \quad \Xi\left(\zeta ; \Gamma_{1}\left(\sigma ; \Delta_{1}, B_{2}, \Delta_{2}\right), A, \Gamma_{2}\right) \rightarrow C}{\Xi\left(\xi, \zeta ; \Gamma_{1}\left(\sigma ; \Delta_{1}, B_{2}, \Delta_{2}\right), \Pi, \Gamma_{2}\right) \rightarrow C} \mathrm{cut}
$$

Again, $\Gamma_{1}$ could coincide with its designated sub-meta-formula; in this case, $\sigma$ gets merged with $\xi, \zeta$.

In both situations, the two new cuts are independent and have a smaller $\sigma$ parameter with the same $\kappa$.

Subcase 4.e (similar to the previous one). The lowermost rule in $\mathscr{D}_{\text {right }}$ is $\wedge R$ :

$$
\frac{\xi ; \Pi \rightarrow A \frac{\Xi\left(\zeta ; \Gamma_{1}, A, \Gamma_{2}\right) \rightarrow C_{1} \quad \Xi\left(\zeta ; \Gamma_{1}, A, \Gamma_{2}\right) \rightarrow C_{2}}{\Xi\left(\zeta ; \Gamma_{1}, A, \Gamma_{2}\right) \rightarrow C_{1} \wedge C_{2}}}{\Xi\left(\xi, \zeta ; \Gamma_{1}, \Pi, \Gamma_{2}\right) \rightarrow C_{1} \wedge C_{2}} \text { cut } \wedge R
$$

transforms into

$$
\frac{\xi ; \Pi \rightarrow A \quad \Xi\left(\zeta ; \Gamma_{1}, A, \Gamma_{2}\right) \rightarrow C_{1}}{\frac{\Xi\left(\xi, \zeta ; \Gamma_{1}, \Pi, \Gamma_{2}\right) \rightarrow C_{1}}{\Xi\left(\xi, \zeta ; \Gamma_{1}, \Pi, \Gamma_{2}\right) \rightarrow C_{1} \wedge C_{2}} \text { cut } \frac{\xi ; \Pi \rightarrow A \quad \Xi\left(\zeta ; \Gamma_{1}, A, \Gamma_{2}\right) \rightarrow C_{2}}{\Xi\left(\xi \zeta ; \Gamma_{1}, \Pi, \Gamma_{2}\right) \rightarrow C_{2}}} \text { cut }
$$

Again, the two new cuts have a smaller $\sigma$ and the same $\kappa$.

Case 5 (axiom). One of the sequents is an axiom of the form $A \rightarrow A$. Then cut disappears, since the other premise coincides with the goal.

Theorem 2 Let sequents $\xi ; \Pi \rightarrow A$ and $\Xi\left(\zeta ; \Gamma_{1}, A, \Gamma_{2} \rightarrow C\right)$ be derivable in $!_{\mathbf{b}}^{\mathbf{2 0 1 5}} \mathbf{M A L C} \mathbf{C}^{*} \mathbf{b}(\mathrm{st})^{\prime}$. Then the sequent $\left.\Xi\left(\xi, \zeta ; \Gamma_{1}, \Pi, \Gamma_{2}\right) \rightarrow C\right)$ is also derivable in $!_{\mathbf{b}}^{\mathbf{2 0 1 5}} \mathbf{M A L C}{ }^{*} \mathbf{b}(\mathrm{st})^{\prime}$.

Proof The $!_{\mathbf{b}}^{\mathbf{2 0 1 5}} \mathbf{M A L C}{ }^{*} \mathbf{b}(\mathrm{st})^{\prime}$ system differs from $!_{\mathrm{b}}^{\mathbf{2 0 1 8}} \mathbf{M A L C} \mathbf{C}^{*} \mathbf{b}(\mathrm{st})^{\prime}$ only in two rules: $! R^{\prime}$ and $! C^{\prime}$. Thus, we have to reconsider Case 1 and Subcase $4 . \mathrm{a}^{\prime}$ (in Subcase 3.a, ! $C^{\prime}$ still acts as an "easy rule").

Case 1. The bottom of $\mathscr{D}_{\text {left }}$ now is

$$
\frac{\xi ; \Lambda \rightarrow A}{\xi ; \Lambda \rightarrow ! A} ! R^{\prime}
$$

with $\xi \neq \varnothing$. We perform the same deep cut elimination procedure, as in Theorem 1. Namely, the lowermost rule application in $\mathscr{D}_{\text {right }}$ is

$$
\frac{\Xi\left(\zeta, A ; \Gamma^{\prime}, \Gamma^{\prime \prime}\right) \rightarrow C}{\Xi\left(\zeta ; \Gamma^{\prime}, ! A, \Gamma^{\prime \prime}\right) \rightarrow C} ! L
$$

and we trace the $A$ in the stoup upwards until applications of $! P$. Next, we replace these occurrences of $A$ with $\xi$.

The active $! P$ applications,

$$
\frac{\Xi_{i}\left(\zeta_{i} ; \Gamma_{i}^{\prime}, A, \Gamma_{i}^{\prime \prime}\right) \rightarrow C_{i}}{\Xi_{i}\left(\zeta_{i}, A ; \Gamma_{i}^{\prime}, \Gamma_{i}^{\prime \prime}\right) \rightarrow C_{i}} ! P
$$


transform into cuts with a smaller $\kappa$ :

$$
\frac{\xi ; \Lambda \rightarrow A \quad \Xi_{i}\left(\zeta_{i} ; \Gamma_{i}^{\prime}, A, \Gamma_{i}^{\prime \prime}\right) \rightarrow C_{i}}{\Xi_{i}\left(\zeta_{i}, \xi ; \Gamma_{i}^{\prime}, \Gamma_{i}^{\prime \prime}\right) \rightarrow C_{i}} \mathrm{cut}
$$

Rule applications along the trace remain valid. Notice that the stoup nonemptiness conditions on $! R^{\prime}$ and $! C^{\prime}$ are maintained by non-emptiness of $\xi$.

Subcase 4. $a^{\prime}$. In this case,

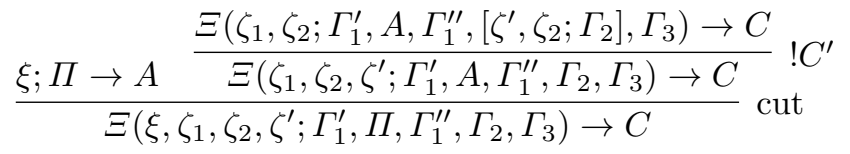

(with $\zeta_{2} \neq \varnothing$ ) transforms into

$$
\frac{\xi ; \Pi \rightarrow A \quad \Xi\left(\zeta_{1}, \zeta_{2} ; \Gamma_{1}^{\prime}, A, \Gamma_{1}^{\prime \prime},\left[\zeta^{\prime}, \zeta_{2} ; \Gamma_{2}\right], \Gamma_{3}\right) \rightarrow C}{\frac{\Xi\left(\xi, \zeta_{1}, \zeta_{2} ; \Gamma_{1}^{\prime}, \Pi, \Gamma_{1}^{\prime \prime},\left[\zeta^{\prime}, \zeta_{2} ; \Gamma_{2}\right], \Gamma_{3}\right) \rightarrow C}{\Xi\left(\xi, \zeta_{1}, \zeta_{2}, \zeta^{\prime} ; \Gamma_{1}^{\prime}, \Pi, \Gamma_{1}^{\prime \prime}, \Gamma_{2}, \Gamma_{3}\right) \rightarrow C} ! C^{\prime}} \mathrm{cut}
$$

(the case with $A$ in $\Gamma_{3}$ is considered symmetrically), and

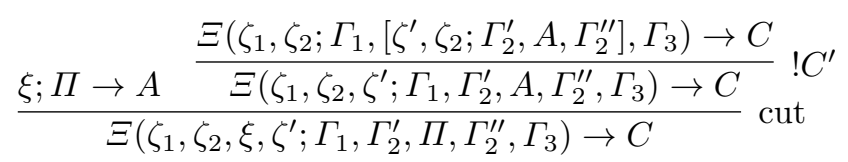

(again, $\zeta_{2} \neq \varnothing$ ) transforms into

$$
\frac{\xi ; \Pi \rightarrow A \quad \Xi\left(\zeta_{1}, \zeta_{2} ; \Gamma_{1},\left[\zeta^{\prime}, \zeta_{2} ; \Gamma_{2}^{\prime}, A, \Gamma_{2}^{\prime \prime}\right], \Gamma_{3}\right) \rightarrow C}{\frac{\Xi\left(\zeta_{1}, \zeta_{2} ; \Gamma_{1},\left[\xi, \zeta^{\prime}, \zeta_{2} ; \Gamma_{2}^{\prime}, \Pi, \Gamma_{2}^{\prime \prime}\right], \Gamma_{3}\right) \rightarrow C}{\Xi\left(\zeta_{1}, \zeta_{2}, \xi, \zeta^{\prime} ; \Gamma_{1}, \Gamma_{2}^{\prime}, \Pi, \Gamma_{2}^{\prime \prime}, \Gamma_{3}\right) \rightarrow C} ! C^{\prime}} \mathrm{cut}
$$

\section{Versions of Modified Morrill's Systems without Stoups}

In this section we provide alternative, in a sense more traditional formulations of $!_{\mathrm{b}}^{\mathbf{2 0 1 5}} \mathrm{MALC} \mathbf{C}^{*} \mathbf{b}(\mathrm{st})^{\prime}, !_{\mathrm{b}}^{\mathbf{2 0 1 8}} \mathbf{M A L C}{ }^{*} \mathbf{b}(\mathrm{st})^{\prime}$, and $!_{\mathrm{b}}^{\mathbf{2 0 1 8}} \mathbf{M A L C b}(\mathrm{st})^{\prime}$ without stoups (like in Morrill and Valentín (2015a)). We denote these calculi by $!_{b}^{2015}$ MALC ${ }^{*} b, !_{b}^{2018} \mathbf{M A L C}^{*} \mathrm{~b}$, and $!_{\mathrm{b}}^{2018} \mathrm{MALCb}$ respectively.

Formulae, meta-formulae, and sequents are defined as in Morrill's system (Section 3), but all the stoups are empty now.

We start with $!_{\mathbf{b}}^{\mathbf{2 0 1 5}} \mathbf{M A L C}{ }^{*} \mathbf{b}$ and $!_{\mathbf{b}}^{\mathbf{2 0 1 8}} \mathbf{M A L C}{ }^{*} \mathbf{b}$. Both calculi are built on top of MALC* $\mathbf{b}$, the multiplicative-additive Lambek calculus with brackets (Morrill, 1992, Moortgat, 1996). Axioms and rules of MALC*b are as follows:

$$
\overline{A \rightarrow A} \text { id }
$$




$$
\begin{aligned}
& \frac{\Gamma \rightarrow B \quad \Xi\left(\Delta_{1}, C, \Delta_{2}\right) \rightarrow D}{\Xi\left(\Delta_{1}, C / B, \Gamma, \Delta_{2}\right) \rightarrow D} / L \quad \frac{\Gamma, B \rightarrow C}{\Gamma \rightarrow C / B} / R \\
& \frac{\Gamma \rightarrow A \quad \Xi\left(\Delta_{1}, C, \Delta_{2}\right) \rightarrow D}{\Xi\left(\Delta_{1}, \Gamma, A \backslash C, \Delta_{2}\right) \rightarrow D} \backslash L \quad \frac{A, \Gamma \rightarrow C}{\Gamma \rightarrow A \backslash C} \backslash R \\
& \frac{\Xi\left(\Delta_{1}, A, B, \Delta_{2}\right) \rightarrow D}{\Xi\left(\Delta_{1}, A \cdot B, \Delta_{2}\right) \rightarrow D} \cdot L \quad \frac{\Delta \rightarrow A \quad \Gamma \rightarrow B}{\Delta, \Gamma \rightarrow A \cdot B} \cdot R \\
& \frac{\Xi\left(\Delta_{1}, \Delta_{2}\right) \rightarrow A}{\Xi\left(\Delta_{1}, \mathbf{1}, \Delta_{2}\right) \rightarrow A} \mathbf{1} L \quad \overline{\mathbf{1}} \mathbf{1} R \\
& \frac{\Xi\left(\Delta_{1}, A, \Delta_{2}\right) \rightarrow B}{\Xi\left(\Delta_{1},\left[[]^{-1} A\right], \Delta_{2}\right) \rightarrow B}[]^{-1} L \quad \frac{[\Xi] \rightarrow A}{\Xi \rightarrow[]^{-1} A}[]^{-1} R \\
& \frac{\Xi\left(\Delta_{1},[A], \Delta_{2}\right) \rightarrow B}{\Xi\left(\Delta_{1},\langle\rangle A, \Delta_{2}\right) \rightarrow B}\langle\rangle L \quad \frac{\Xi \rightarrow A}{[\Xi] \rightarrow\langle\rangle A}\langle\rangle R
\end{aligned}
$$

The following rules for ! are the same in both systems, $!_{\mathbf{b}}^{\mathbf{2 0 1 5}} \mathbf{M A L C} \mathbf{C}^{*} \mathbf{b}$ and $!_{\mathrm{b}}^{2018} \mathrm{MALC}^{*} \mathrm{~b}$ :

$$
\begin{gathered}
\frac{\Xi\left(\Delta_{1}, A, \Delta_{2}\right) \rightarrow C}{\Xi\left(\Delta_{1}, ! A, \Delta_{2}\right) \rightarrow C} ! L \\
\frac{\Xi\left(\Delta_{1}, ! A, \Phi, \Delta_{2}\right) \rightarrow C}{\Xi\left(\Delta_{1}, \Phi, ! A, \Delta_{2}\right) \rightarrow C} ! P_{1} \quad \frac{\Xi\left(\Delta_{1}, \Phi, ! A, \Delta_{2}\right) \rightarrow C}{\Xi\left(\Delta_{1}, ! A, \Phi, \Delta_{2}\right) \rightarrow C} ! P_{2}
\end{gathered}
$$

The difference between $!_{\mathbf{b}}^{\mathbf{2 0 1 5}} \mathbf{M} \mathbf{A} \mathbf{L} \mathbf{C}^{*} \mathbf{b}$ and $!_{\mathbf{b}}^{\mathbf{2 0 1 8}} \mathbf{M} \mathbf{A} \mathbf{L} \mathbf{C}^{*} \mathbf{b}$ is in the $! R$ and $! C$ rules. In $!_{\mathbf{b}}^{\mathbf{2 0 1 5}} \mathbf{M A L} \mathbf{C}^{*} \mathbf{b}$, they are formulated as follows

$$
\begin{gathered}
\frac{! A_{1}, \ldots, ! A_{n} \rightarrow B}{! A_{1}, \ldots, ! A_{n} \rightarrow ! B} ! R, n \geq 1 \\
\frac{\Xi\left(! A_{1}, \ldots, ! A_{n}, \Gamma_{1},\left[! A_{1}, \ldots, ! A_{n}, \Gamma_{2}\right], \Gamma_{3}\right) \rightarrow C}{\Xi\left(! A_{1}, \ldots, ! A_{n}, \Gamma_{1}, \Gamma_{2}, \Gamma_{3}\right) \rightarrow C} ! C, n \geq 1
\end{gathered}
$$

For $!_{\mathbf{b}}^{\mathbf{2 0 1 8}} \mathbf{M A L C} \mathbf{C}^{*} \mathbf{b}$, these rules are formulated as follows:

$$
\frac{! A \rightarrow B}{! A \rightarrow ! B} ! R \quad \frac{\Xi\left(! A, \Gamma_{1},\left[! A, \Gamma_{2}\right], \Gamma_{3}\right) \rightarrow C}{\Xi\left(! A, \Gamma_{1},\left[\left[\Gamma_{2}\right]\right], \Gamma_{3}\right) \rightarrow C} ! C
$$

The cut rule of all stoup-free calculi is formulated as follows:

$$
\frac{\Pi \rightarrow A \quad \Xi\left(\Gamma_{1}, A, \Gamma_{2}\right) \rightarrow C}{\Xi\left(\Gamma_{1}, \Pi, \Gamma_{2}\right) \rightarrow C} \text { cut }
$$

In order to obtain $!_{\mathbf{b}}^{\mathbf{2 0 1 8}} \mathbf{M A L C b}$, we impose Lambek's restriction on the rules of $!_{\mathrm{b}}^{\mathbf{2 0 1 8}} \mathbf{M A L C}{ }^{*} \mathbf{b}$ in the following natural way: 
- in $\backslash R$ and $/ R$, we require $\Gamma \neq \Lambda$;

- in $! C$, we require $\Gamma_{2} \neq \Lambda$.

Proposition 1 Let $\Xi \rightarrow C$ be a sequent without stoups (in the context of $!_{\mathrm{b}}^{2018} \mathbf{M A L C}{ }^{*} \mathbf{b}(\mathrm{st})^{\prime}$ we consider it as a sequent with empty stoups). Then the following are equivalent:

1. $\Xi \rightarrow C$ is derivable in $!_{\mathrm{b}}^{2018} \mathbf{M A L C} \mathbf{C}^{*} \mathbf{b}$ without cut;

2. $\Xi \rightarrow C$ is derivable in $!_{\mathrm{b}}^{\mathbf{2 0 1 8}} \mathbf{M A L C} \mathbf{C}^{*} \mathbf{b}$, possibly using cut;

3. $\Xi \rightarrow C$ is derivable in $!_{\mathbf{b}}^{\mathbf{2 0 1 8}} \mathbf{M A L C} \mathbf{C}^{*} \mathbf{b}(\mathrm{st})^{\prime}$, possibly using cut;

4. $\Xi \rightarrow C$ is derivable in $!_{\mathbf{b}}^{\mathbf{2 0 1 8}} \mathbf{M A L C} \mathbf{C}^{*} \mathbf{b}(\mathrm{st})^{\prime}$ without cut.

The same holds for the variants with Lambek's restriction, $!_{\mathbf{b}}^{2018} \mathbf{M A L C b}(\mathrm{st})^{\prime}$ and $!_{\mathrm{b}}^{2018} \mathrm{MALCb}$.

Proof We proceed by round-robin implications: $1 \Rightarrow 2 \Rightarrow 3 \Rightarrow 4 \Rightarrow 1$.

$1 \Rightarrow 2$ Obvious.

$2 \Rightarrow 3$ Consider a derivation of $\Xi \rightarrow C$ in $!_{\mathbf{b}}^{2018} \mathbf{M A L C} \mathbf{C}^{*} \mathbf{b}$ (possibly with cuts) and translate it into $!_{\mathbf{b}}^{\mathbf{2 0 1 8}} \mathbf{M A L C} \mathbf{C}^{*} \mathbf{b}(\mathrm{st})$. The interesting case concerns !-operating rules. These rules are simulated using cut, by temporarily moving the active !-formula to the stoup (which is normally empty):

$$
\begin{aligned}
& \frac{A ; \Lambda \rightarrow ! A \quad \Xi\left(\Gamma_{1}, \Phi, ! A, \Gamma_{2}\right) \rightarrow C}{\frac{\Xi\left(A ; \Gamma_{1}, \Phi, \Gamma_{2}\right) \rightarrow C}{\Xi\left(\Gamma_{1}, ! A, \Phi, \Gamma_{2}\right) \rightarrow C} ! L} \text { cut }
\end{aligned}
$$

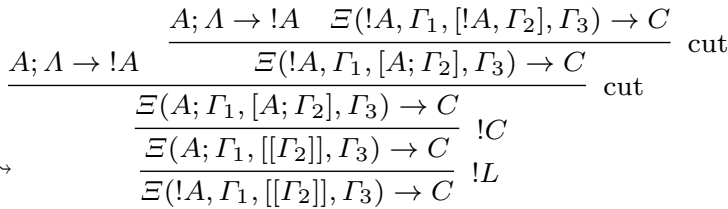

$$
\begin{aligned}
& \frac{\Xi\left(! A, \Gamma_{1},\left[! A, \Gamma_{2}\right], \Gamma_{3}\right) \rightarrow C}{\Xi\left(! A, \Gamma_{1},\left[\left[\Gamma_{2}\right]\right], \Gamma_{3}\right) \rightarrow C} ! C \leadsto \quad \frac{\overline{\Xi\left(A ; \Gamma_{1},\left[\left[\Gamma_{2}\right]\right], \Gamma_{3}\right) \rightarrow C} !}{\Xi\left(! A, \Gamma_{1},\left[\left[\Gamma_{2}\right]\right], \Gamma_{3}\right) \rightarrow C} ! L
\end{aligned}
$$

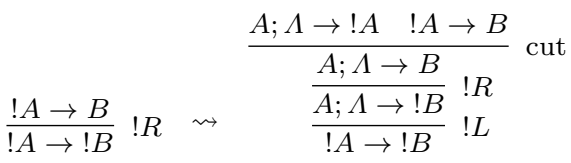

$$
\begin{aligned}
& \frac{\Xi\left(\Gamma_{1}, A, \Gamma_{2}\right) \rightarrow C}{\Xi\left(\Gamma_{1}, ! A, \Gamma_{2}\right) \rightarrow C} ! L \rightsquigarrow \frac{\frac{\Xi\left(\Gamma_{1}, A, \Gamma_{2}\right) \rightarrow C}{\Xi\left(A ; \Gamma_{1}, \Gamma_{2}\right) \rightarrow C} ! P}{\Xi\left(\Gamma_{1}, ! A, \Gamma_{2}\right) \rightarrow C} ! L
\end{aligned}
$$

The sequent $A ; \Lambda \rightarrow ! A$ is derived as follows:

$$
\frac{A \rightarrow A}{\frac{A ; \Lambda \rightarrow A}{A ; \Lambda \rightarrow ! A} ! R} ! R
$$

All other rules, including cut, are translated straightforwardly. 
$3 \Rightarrow 4$ This is due to cut elimination (Theorem 1).

$4 \Rightarrow 1$ Consider a cut-free proof of $\Xi \rightarrow C$ in $!_{\mathbf{b}}^{2018} \mathbf{M A L C} \mathbf{A L}^{*} \mathbf{b}(\mathrm{st})^{\prime}$. In the goal sequent, all stoups are empty, but this could be not the case for sequents inside the derivation. In each sequent, we "flatten" the stoups, replacing each meta-formula $\zeta ; \Gamma$, where $\zeta=\left\{A_{1}, \ldots, A_{N}\right\}$, with $! A_{1}, \ldots, ! A_{N}, \Gamma$. For $\zeta=$ $\left\{A_{1}, \ldots, A_{N}\right\}$, let us denote $! A_{1}, \ldots, ! A_{N}$ by $! \zeta$.

The rules of $!_{\mathrm{b}}^{\mathbf{2 0 1 8}} \mathbf{M A L C}^{*} \mathbf{b}(\mathrm{st})^{\prime}$ which do not operate the stoup are mapped to those of $!_{\mathrm{b}}^{2018}$ MALC ${ }^{*} \mathbf{b}$, adding permutations for !-formulae, if necessary. For example, this is how it is performed for $\backslash R$ and $\backslash L$ :

$$
\begin{aligned}
\frac{\zeta ; B, \Gamma \rightarrow C}{\zeta ; \Gamma \rightarrow B \backslash C} \backslash R & \rightsquigarrow \frac{\frac{! \zeta, B, \Gamma \rightarrow C}{\overline{B, ! \zeta, \Gamma \rightarrow C} ! P}}{! \zeta, \Gamma \rightarrow B \backslash C} \backslash R \\
\frac{\zeta_{1} ; \Gamma \rightarrow B \quad \Xi\left(\zeta_{2} ; \Delta_{1}, C, \Delta_{2}\right) \rightarrow D}{\Xi\left(\zeta_{1}, \zeta_{2} ; \Delta_{1}, \Gamma, B \backslash C, \Delta_{2}\right) \rightarrow D} \backslash L \rightsquigarrow & \frac{\frac{! \zeta_{1}, \Gamma \rightarrow B \quad \Xi\left(! \zeta_{2}, \Delta_{1}, C, \Delta_{2}\right) \rightarrow D}{\Xi\left(! \zeta_{2}, \Delta_{1}, ! \zeta_{1}, \Gamma, B \backslash C, \Delta_{2}\right) \rightarrow C}}{\overline{\left.\Xi ! ! \zeta_{1}, ! \zeta_{2}, \Delta_{1}, \Gamma, B \backslash C, \Delta_{2}\right) \rightarrow D} ! P} \backslash L
\end{aligned}
$$

Notice how Lambek's restriction is conserved in the $\backslash R$ rule.

Contraction is handled as follows:

$$
\begin{aligned}
& \frac{\Xi\left(! \zeta, ! A, \Gamma_{2},\left[! \zeta^{\prime}, ! A, \Gamma_{2}\right], \Gamma_{3}\right) \rightarrow B}{\Xi\left(! A, ! \zeta, \Gamma_{2},\left[! \zeta^{\prime}, ! A, \Gamma_{2}\right], \Gamma_{3}\right) \rightarrow B} ! P \\
& \frac{\Xi\left(\zeta, A ; \Gamma_{1},\left[\zeta^{\prime}, A ; \Gamma_{2}\right], \Gamma_{3}\right) \rightarrow B}{\Xi\left(\zeta, A ; \Gamma_{1},\left[\left[\zeta^{\prime} ; \Gamma_{2}\right]\right], \Gamma_{3}\right) \rightarrow B} ! C^{\prime} \rightsquigarrow \frac{\frac{\Xi\left(! A, ! \zeta, \Gamma_{1},\left[! A, ! \zeta^{\prime}, \Gamma_{2}\right], \Gamma_{3}\right) \rightarrow B}{\Xi\left(! A, ! \zeta, \Gamma_{1},\left[\left[! \zeta^{\prime}, \Gamma_{2}\right]\right], \Gamma_{3}\right) \rightarrow B} ! C}{\Xi\left(! \zeta, ! A, \Gamma_{1},\left[\left[! \zeta^{\prime}, \Gamma_{2}\right]\right], \Gamma_{3}\right) \rightarrow B} ! P
\end{aligned}
$$

Again, we notice that Lambek's restriction $\left(! \zeta_{2}, \Gamma_{2} \neq \Lambda\right)$ is conserved.

Finally, $! L$ becomes just permutation, $! R^{\prime}$ maps to $! R$, and the version of $! P$ in $!_{\mathbf{b}}^{\mathbf{2 0 1 8}} \mathbf{M A L} \mathbf{C}^{*} \mathbf{b}(\mathrm{st})^{\prime}$ maps to a combination of permutation and $! L$ of $!_{\mathrm{b}}^{2018} \mathrm{MALC}^{*} \mathrm{~b}$.

Proposition 2 Let $\Xi \rightarrow C$ be a sequent without stoups (in the context of $!_{\mathbf{b}}^{\mathbf{2 0 1 5}} \mathbf{M A L C} \mathbf{C}^{*} \mathbf{b}(\mathrm{st})^{\prime}$ we consider it as a sequent with empty stoups). Then the following are equivalent:

1. $\Xi \rightarrow C$ is derivable in $!_{\mathbf{b}}^{\mathbf{2 0 1 5}} \mathbf{M A L C} \mathbf{L}^{*} \mathbf{b}$ without cut;

2. $\Xi \rightarrow C$ is derivable in $!_{\mathrm{b}}^{\mathbf{2 0 1 5}} \mathbf{M A L C} \mathbf{C}^{*} \mathbf{b}$, possibly using cut;

3. $\Xi \rightarrow C$ is derivable in $!_{\mathbf{b}}^{\mathbf{2 0 1 5}} \mathbf{M A L C} \mathbf{C}^{*} \mathbf{b}(\mathrm{st})^{\prime}$, possibly using cut;

4. $\Xi \rightarrow C$ is derivable in $!_{\mathrm{b}}^{\mathbf{2 0 1 5}} \mathbf{M A L C} \mathbf{C}^{*} \mathbf{b}(\mathrm{st})^{\prime}$ without cut.

Proof $1 \Rightarrow 2$ Obvious. 
$2 \Rightarrow 3$ The $! R$ and $! C$ rules of $!_{\mathbf{b}}^{\mathbf{2 0 1 5}} \mathbf{M A L C} \mathbf{C}^{*} \mathbf{b}$ are simulated in the system $!_{\mathrm{b}}^{2015} \mathrm{MALC}^{*} \mathbf{b}(\mathrm{st})^{\prime}$ as follows:

$$
\begin{aligned}
& \underline{A_{n} ; \Lambda \rightarrow ! A_{n} \quad ! A_{1}, \ldots, ! A_{n} \rightarrow B} \text { cut } \\
& \frac{A_{1} ; \Lambda \rightarrow ! A \quad \overline{A_{2}, \ldots, A_{n} ; ! A_{1} \rightarrow B}}{A_{1}, \ldots, A_{n} ; \Lambda \rightarrow B} \text { cut } \\
& \frac{! A_{1}, \ldots, ! A_{n} \rightarrow B}{! A_{1}, \ldots, ! A_{n} \rightarrow ! B} ! R, n \geq 1 \rightsquigarrow \quad \rightsquigarrow \quad \frac{\overline{A_{1}, \ldots, A_{n} ; \Lambda \rightarrow ! B} ! R^{\prime},\left\{A_{1}, \ldots, A_{n}\right\} \neq \varnothing}{\frac{A_{1}, \ldots, ! A_{n} \rightarrow ! B}{! A_{1}, \ldots}} \\
& \frac{\Xi\left(! A_{1}, \ldots, ! A_{n}, \Gamma_{1},\left[! A_{1}, \ldots, ! A_{n}, \Gamma_{2}\right], \Gamma_{3}\right) \rightarrow C}{\Xi\left(! A_{1}, \ldots, ! A_{n}, \Gamma_{1}, \Gamma_{2}, \Gamma_{3}\right) \rightarrow C} ! C, n \geq 1 \\
& \underline{A_{n} ; \Lambda \rightarrow ! A_{n} \quad \Xi\left(! A_{1}, \ldots, ! A_{n}, \Gamma_{1},\left[! A_{1}, \ldots, ! A_{n}, \Gamma_{2}\right], \Gamma_{3}\right) \rightarrow C} \text { cut }
\end{aligned}
$$

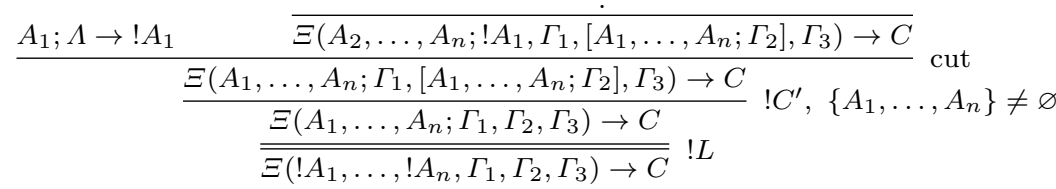

where $A_{i} ; \Lambda \rightarrow ! A_{i}(i=1, \ldots, n)$ is derived as follows:

$$
\frac{\frac{A_{i} \rightarrow A_{i}}{A_{i} ; \Lambda \rightarrow A_{i}} ! P}{A_{i} ; \Lambda \rightarrow ! A_{i}} ! R,\left\{A_{i}\right\} \neq \varnothing
$$

All other rules are translated exactly as in the proof of the $2 \Rightarrow 3$ implication of Proposition 1.

$3 \Rightarrow 4$ This is due to cut eliminaton, Theorem 2

$4 \Rightarrow 1$ The $! R^{\prime}$ rule of $!_{\mathbf{b}}^{\mathbf{2 0 1 5}} \mathbf{M A L C}{ }^{*} \mathbf{b}(\text { st })^{\prime}$ maps directly onto the $! R$ rule of $!_{\mathrm{b}}^{\mathbf{2 0 1 5}} \mathbf{M A L C}{ }^{*} \mathbf{b}$. Contraction is handled as follows:

$$
\frac{\Xi\left(\zeta_{1}, \zeta_{2} ; \Gamma_{1},\left[\zeta^{\prime}, \zeta_{2} ; \Gamma_{2}\right], \Gamma_{3}\right) \rightarrow C}{\Xi\left(\zeta_{1}, \zeta_{2}, \zeta^{\prime} ; \Gamma_{1}, \Gamma_{2}, \Gamma_{3}\right) \rightarrow C} ! C^{\prime}, \zeta_{2} \neq \varnothing
$$

transforms into

$$
\frac{\frac{\Xi\left(! \zeta_{1}, ! \zeta_{2}, \Gamma_{1},\left[! \zeta^{\prime}, ! \zeta_{2}, \Gamma_{2}\right], \Gamma_{3}\right) \rightarrow C}{\overline{\Xi\left(! \zeta_{2}, ! \zeta_{1}, \Gamma_{1},\left[! \zeta_{2}, ! \zeta^{\prime}, \Gamma_{2}\right], \Gamma_{3}\right) \rightarrow C}} ! P}{\frac{\Xi\left(! \zeta_{2}, ! \zeta_{1}, \Gamma_{1}, ! \zeta^{\prime}, \Gamma_{2}, \Gamma_{3}\right) \rightarrow C}{\overline{\Xi\left(! \zeta_{1}, ! \zeta_{2}, ! \zeta^{\prime}, \Gamma_{1}, \Gamma_{2}, \Gamma_{3}\right) \rightarrow C}} ! P, ! \zeta_{2} \neq \Lambda}
$$

All other rules are translated exactly as in the proof of the $4 \Rightarrow 1$ implication of Proposition 1.

Finally, for $!_{\mathbf{b}}^{\mathbf{2 0 1 8}}$ MALC* $\mathbf{b}($ st) we prove only one implication, since the other one does not hold (for counter-example see Section 4 ). 
Proposition 3 If a sequent without stoups is derivable in $!_{\mathbf{b}}^{\mathbf{2 0 1 8}} \mathbf{M A L C} \mathbf{A} \mathbf{b}(\mathrm{st})$, then it is also derivable in $\mathbf{!}_{\mathbf{b}}^{\mathbf{2 0 1 8}} \mathbf{M A L C} \mathbf{C}^{*} \mathbf{b}$.

Proof Recall that derivations in $!_{\mathbf{b}}^{\mathbf{2 0 1 8}} \mathbf{M A L C} \mathbf{M}^{*} \mathbf{b}(\mathrm{st})$ are cut-free by definition. Thus, this proposition can be proved by modifying the $4 \Rightarrow 1$ implication of Proposition 1. The rules that are different in $!_{\mathbf{b}}^{\mathbf{2 0 1 8}} \mathbf{M A L C} \mathbf{C}^{*} \mathbf{b}(\mathrm{st})$, if compared with $!_{\mathbf{b}}^{2018} \mathbf{M A L C}{ }^{*} \mathbf{b}(\mathrm{st})^{\prime}$, are $! R$ and $! C$. Thus, we need to reconsider these rules. Fortunately, after "flattening" the stoups, $! R$ and $! C$ become identical to $! R^{\prime}$ and $! C^{\prime}$ respectively.

\section{The $\pi$ and $\pi_{q}$ Projections}

The calculi presented above are related to the bracket-free system $\mathbf{M} \mathbf{M} \mathbf{A L} \mathbf{C}^{*}$ by means of so-called bracket-forgetting projections (BFP). The BFPs are going to be used in the undecidability proofs (Section 9 below), in order to make use of the standard undecidability proof for !MALC* in the undecidability proofs for more sophisticated sequents with brackets. We define two versions of BFP, and for simplicity we do this for the syntax without stoups.

Definition 2 The $\pi$-projection of a formula is defined in the following recursive way:

$$
\begin{array}{ll}
\pi(p)=p \text { for } p \in \operatorname{Var} & \pi(A \cdot B)=\pi(A) \cdot \pi(B) \\
\pi(A \backslash B)=\pi(A) \backslash \pi(B) & \pi(B / A)=\pi(B) / \pi(A) \\
\pi\left(A_{1} \wedge A_{2}\right)=\pi\left(A_{1}\right) \wedge \pi\left(A_{2}\right) & \pi\left(A_{1} \vee A_{2}\right)=\pi\left(A_{1}\right) \vee \pi\left(A_{2}\right) \\
\pi(! A)=! \pi(A) & \pi(\mathbf{1})=\mathbf{1} \\
\pi\left([]^{-1} A\right)=\pi(A) & \pi(\langle\rangle A)=\pi(A)
\end{array}
$$

For meta-formulae (tree terms) and sequents without stoups the $\pi$-projection is defined as follows:

$$
\begin{array}{ll}
\pi\left(\Gamma_{1}, \ldots, \Gamma_{k}\right)=\pi\left(\Gamma_{1}\right), \ldots, \pi\left(\Gamma_{k}\right) & \pi(\Lambda)=\Lambda \\
\pi([\Xi])=\pi(\Xi) & \pi(\Xi \rightarrow C)=(\pi(\Xi) \rightarrow \pi(C))
\end{array}
$$

For technical reasons we shall also need the following modification of $\pi$ projection.

Definition 3 Let $q$ be a designated variable. Then the $\pi_{q}$-projection is defined on variables as follows:

$$
\pi_{q}(p)= \begin{cases}p, & \text { if } p \neq q \\ \mathbf{1}, & \text { if } p=q\end{cases}
$$

and then propagated to formulae, meta-formulae, and sequents exactly as $\pi$. 
The $\pi$-projection erases all bracket information from a sequent. Since brackets block some unwanted derivabilities, this projection is only one-way sound, as formulated in the following definition. The $\pi_{q}$-projection additionally makes the special variable $q$ behave as a unit (this is going to be necessary when handling $!_{\mathbf{b}}^{\mathbf{2 0 1 8}} \mathbf{M A L C b}(\mathrm{st})$, the system with Lambek's restriction which does not include a unit constant).

Definition 4 A calculus $\mathcal{L}$ is $\pi$-sound $\left(\pi_{q}\right.$-sound) in !MALC $^{*}$ if for any sequent derivable in $\mathcal{L}$ its $\pi$-projection (resp., $\pi_{q}$-projection) is derivable in !MALC*

Since the rules of all Morrill's systems (in the version without stoups), after applying the $\pi$-projection, map to rules of !MALC ${ }^{*}$, we automatically get $\pi$ soundness for Morrill's systems. For $\pi_{q}$-soundness, we additionally notice that the axiom $q \rightarrow q$ maps to a derivable sequent $\mathbf{1} \rightarrow \mathbf{1}$ (everything else remains as for the $\pi$-projection).

Proposition 4 The following calculi are $\pi$-sound and $\pi_{q}$-sound in !MALC* $!_{\mathrm{b}}^{2015} \mathrm{MALC}^{*} \mathrm{~b}, !_{\mathrm{b}}^{2018} \mathrm{MALC}{ }^{*} \mathrm{~b}$, and $!_{\mathrm{b}}^{2018} \mathrm{MALCb}$.

Notice that $\pi$ and $\pi_{q}$ lose essential information about bracketing, so the reverse implication, " $\pi$-completeness" or " $\pi_{q}$-completeness," does not (and is not intended to) hold. For example, \langle\rangle $p \rightarrow p$ is not derivable in any of the Morrill's systems, while its $\pi$-projection (and also $\pi_{q}$-projection), $p \rightarrow p$, is an axiom of !MALC ${ }^{*}$. More interesting examples arise from the linguistic usage of brackets (see Linguistic Introduction): e.g., * "the girl whom John loves Mary and Pete loves" is not assigned type NP (parsed as a valid noun phrase) in bracketed calculi, but after forgetting the brackets and bracket modalities the corresponding sequent becomes derivable in !MALC*.

For fragments without additives, there are analogous notions of $\pi$-soundness and $\pi_{q}$-soundness of a given calculus in $! \mathbf{L}^{*}$.

\section{Undecidability}

In this section we prove algorithmic undecidability of the derivability problems for systems defined above. In order to make our results stronger, we confine ourselves to fragments of these systems which include only multiplicative connectives (product and divisions), brackets and bracket modalities, and the subexponential, but not additive connectives. For the full MALC*-variants of the calculi, the corresponding undecidability results follow as corollaries, by conservativity.

Recall the well-known proof of undecidability for $! \mathbf{L}^{*}$, the Lambek calculus (without brackets) enriched with a full-power exponential modality (Lincoln et al., 1992, Kanazawa, 1999, Kanovich et al., 2019a), via encoding of semiThue systems.

A semi-Thue system (Thue, 1914) is a pair $\mathcal{S}=\langle\mathfrak{A}, P\rangle$, where $\mathfrak{A}$ is a finite alphabet and $P$ is a finite set of rewriting rules of the form $\alpha \Rightarrow \beta$, where 
$\alpha$ and $\beta$ are words (possibly empty) over $\mathfrak{A}$. A rule from $P$ can be applied as follows: $\eta \alpha \theta \Rightarrow{ }_{\mathcal{S}} \eta \beta \theta$, where $(\alpha \Rightarrow \beta) \in P$ and $\eta$ and $\theta$ are arbitrary (possibly empty) words over $\mathfrak{A}$. By $\Rightarrow_{\mathcal{S}}^{*}$ (rewritability relation in $\mathcal{S}$ ) we denote the reflexive-transitive closure of $\Rightarrow_{\mathcal{S}}$.

We use the following classical result by Markov (1947) and Post (1947):

Theorem 3 There exists a semi-Thue system $\mathcal{S}$ for which the $\Rightarrow_{\mathcal{S}}^{*}$ relation is algorithmically undecidable, i.e., there exists no algorithm that, given words $\gamma$ and $\delta$, decides whether $\gamma \Rightarrow_{\mathcal{S}}^{*} \delta$.

Before going into the details of our undecidability proof, we sketch the ideas. As noticed by Buszkowski (1982, 2005), semi-Thue systems can be encoded as finite theories (that is, sets of sequents considered as additional axioms) over the Lambek calculus. Following Buszkowski (2005), such an encoding can performed in a very natural way, by taking a new axiom $y_{1}, \ldots, y_{m} \rightarrow$ $x_{1} \cdot \ldots x_{k}$ for each rule $x_{1} \ldots x_{k} \Rightarrow y_{1} \ldots y_{m}$ of the semi-Thue system. (Notice how the arrows change their direction here.)

Proposition 5 The sequent $a_{1}, \ldots, a_{n} \rightarrow b_{1} \cdot \ldots \cdot b_{k}$ is derivable in MALC* extended with the set of new axioms produced from rules of a semi-Thue system $\mathcal{S}$ (as explained above) if and only if $b_{1} \ldots b_{k} \Rightarrow_{\mathcal{S}}^{*} a_{1} \ldots a_{n}$.

By Theorem 3, this yields undecidability for the problem of derivability from finite sets of hypotheses in the Lambek calculus.

In his earlier paper, Buszkowski (1982) provides a much more sophisticated encoding of semi-Thue derivations using only one division operation (the encoding above also uses product). We discuss this encoding later, in Section 11 .

Reducing derivability from finite theories to "pure" derivability requires a sort of deduction theorem, which allows to internalise additional axioms (hypotheses) into the sequent being derived. For classical or intuitionistic logic, for example, this could be implemented as follows: formula $\varphi$ is derivable from hypotheses $\psi_{1}, \ldots, \psi_{n}$ if and only if the formula $\psi_{1} \rightarrow\left(\psi_{2} \rightarrow \ldots \rightarrow\right.$ $\left.\left(\psi_{n} \rightarrow \varphi\right) \ldots\right)$ is derivable without hypotheses. In the Lambek calculus without (sub)exponentials, however, such a theorem is impossible, due to the substructural nature of the system. This is due to the fact that in derivations from hypotheses each hypothesis can be used several (but also maybe zero) times, while in the absence of weakening and contraction the "pure" calculus treats each formula as a "resource" which should be used exactly once.

The full-power exponential, as in !MALC* ${ }^{*}$, enables the structural rules of weakening, contraction, and permutation, and enjoys internalisation of extra axiom, in the following form.

Proposition 6 A sequent $\Delta \rightarrow D$ is derivable in !MALC $^{*}$ from a set of sequents $\left\{\Gamma_{1} \rightarrow C_{1}, \ldots, \Gamma_{N} \rightarrow C_{N}\right\}$ (possibly using cut) if and only if the sequent ! $\left(C_{1} / \prod \Gamma_{1}\right), \ldots, !\left(C_{N} / \prod \Gamma_{N}\right), \Delta \rightarrow D$ is derivable in !MALC*.

By $\prod \Gamma$, for $\Gamma=E_{1}, \ldots, E_{k}$, we here and further denote the product $E_{1} \cdot \ldots \cdot E_{k}$. Theorem 3 and Propositions 5 and 6 together yield undecidability for $\mathbf{L}^{*}$. 
Subexponentials, with restricted sets of structural rules, also allow internalisation, but the !-formulae used in order to obtain it are more complicated. For ${ }^{\mathbf{r}} \mathbf{L}^{*}$, this is performed by Kanovich et al. (2019a) (Kanovich et al. (2016b) use a slightly different strategy). We perform internalisation of finite sets of hypotheses in Morill's systems, where! interacts with brackets.

For our undecidability proofs, it will be convenient to use Chomsky's type-0 (unrestricted) grammars (Chomsky, 1956), a formalism closely related to semiThue systems. A type-0 grammar $\mathcal{G}$ can be defined as a semi-Thue system $\mathcal{S}$ with the following additional features:

- a designated symbol $s \in \mathfrak{A}$, called the starting symbol;

- a designated subset $\Sigma \subset \mathfrak{A}$, called the terminal alphabet;

- left-hand sides of rewriting rules are required to be non-empty.

The language generated by the type- 0 grammar $\mathcal{G}$ is defined as the set of all words $w$ over the terminal alphabet $\Sigma$, such that $s \Rightarrow_{\mathcal{S}}^{*} w$, where $\mathcal{S}$ is the rewriting (semi-Thue) system of $\mathcal{G}$. Further we use the notation $\Rightarrow_{\mathcal{G}}^{*}$ instead of $\Rightarrow_{\mathcal{S}}^{*}$.

For type-0 grammars, there is the following form of Theorem 3

Theorem 4 There exists a type-0 grammar $\mathcal{G}$ such that the language generated by $\mathcal{G}$ is algorithmically undecidable, i.e., there exists no algorithm that, given a word $w$ over $\Sigma$, decides whether $s \Rightarrow_{\mathcal{G}}^{*} w$.

Wishing to prove undecidability for several closely related calculi, we first introduce an abstract notion of internalisation of finite theories in a deductiontheorem style. Then we prove undecidability for an arbitrary calculus $\mathcal{L}$ which enjoys this property and is $\pi_{q^{-}}$sound in $\mathbf{~} \mathbf{L}^{*}$. The internalisation property facilitates the "forward" direction of the encoding, from a type-0 grammar (semiThue system) to $\mathcal{L}$, and $\pi_{q}$-soundness is used for the "backwards" direction, from derivations in $\mathcal{L}$, via $! \mathbf{L}^{*}$, back to derivations in the grammar.

For simplicity, we are going to internalise formulae rather than sequents: recall that $\Gamma \rightarrow C$ corresponds to $C / \prod \Gamma$. Further we shall designate two specific variables, $s$ and $q$. The $s$ variable is going to be the starting symbol of the grammar, and $q$ is a fresh variable which should not appear in the grammar. The $q$ variable will be used in lieu of $\mathbf{1}$, since the latter could be unavailable in presence of Lambek's restriction.

Definition 5 Let $\mathcal{A}=\left\{A_{1}, \ldots, A_{N}\right\}$ be a finite set of formulae. A metaformula $\Phi$ internalises $\mathcal{A}$ in the calculus $\mathcal{L}$, if the following holds:

1. the sequent $\Phi, s \rightarrow s$ is derivable in $\mathcal{L}$;

2. the following 'landing' rule is admissible in $\mathcal{L}$ :

$$
\frac{\Phi, \Delta_{1}, A_{i}, \Delta_{2} \rightarrow C}{\Phi, \Delta_{1}, \Delta_{2} \rightarrow C} \text { land, } A_{i} \in \mathcal{A}
$$

3. the sequent $! A_{1}, \ldots, ! A_{N} \rightarrow \prod \pi_{q}(\Phi)$ is derivable in $! \mathbf{L}^{*}$. 
If for any finite set $\mathcal{A}$ of Lambek formulae there exists a meta-formula $\Phi$ which internalises $\mathcal{A}$ in $\mathcal{L}$, then we say that $\mathcal{L}$ internalises finite sets of Lambek formulae.

Let $\mathcal{G}$ be a type- 0 grammar and suppose that all letters of its alphabet are variables $(\mathfrak{A} \subset$ Var $)$. We also introduce an extra fresh variable $q \notin \mathfrak{A}$. Let

$\mathcal{A}_{\mathcal{G}}=\left\{\left(x_{1} \ldots x_{k}\right) /\left(y_{1} \ldots \cdot y_{m}\right) \mid x_{1} \ldots x_{k} \Rightarrow y_{1} \ldots y_{m}\right.$ is a rewriting rule of $\left.\mathcal{G}\right\}$

By definition of a type-0 grammar, $x_{1} \ldots x_{k}$ is always non-empty. On the other hand, $y_{1} \ldots y_{m}$ could be empty $(m=0)$, and in this case we include just $x_{1} \cdot \ldots \cdot x_{k}$ into $\mathcal{B}_{\mathcal{G}}$. Such a rule, with an empty right-hand side, is called an $\varepsilon$-rule and written as $x_{1} \ldots x_{k} \Rightarrow \varepsilon$ ( $\varepsilon$ stands for the empty word).

Now we are ready to formulate and prove the key lemma.

Lemma 1 Let $\mathcal{L}$ be $\pi_{q}$-sound in $! \mathbf{L}^{*}$ and admit the $\cdot L, \cdot R$, and / L rules. Let $\Phi_{\mathcal{G}}$ internalise $\mathcal{A}_{\mathcal{G}}=\left\{A_{1}, \ldots, A_{N}\right\}$ in $\mathcal{L}$ in the sense of Definition 5 . Then the following are equivalent:

1. $s \Rightarrow_{\mathcal{G}}^{*} a_{1} \ldots a_{n}$

2. the sequent $\Phi_{\mathcal{G}}, a_{1}, \ldots, a_{n} \rightarrow s$ is derivable in $\mathcal{L}$;

3. there exists such a bracketing $\Delta$ of $a_{1}, \ldots, a_{n}$ that the sequent $\Phi_{\mathcal{G}}, \Delta \rightarrow s$ is derivable in $\mathcal{L}$;

4. the sequent $! A_{1}, \ldots, ! A_{N}, a_{1}, \ldots, a_{n} \rightarrow s$ is derivable in $! \mathbf{L}^{*}$.

Proof We prove round-robin implications: $1 \Rightarrow 2 \Rightarrow 3 \Rightarrow 4 \Rightarrow 1$.

$1 \Rightarrow 2$ Proceed by induction on the number of rewriting steps in $\Rightarrow_{\mathcal{G}}^{*}$.

The base case is no rewriting steps, $s \Rightarrow_{\mathcal{G}}^{*} s$, and $\Phi_{\mathcal{G}}, s \rightarrow s$ is derivable by Definition 5, item 1 .

Next, let $a_{1} \ldots a_{i} x_{1} \ldots x_{m} a_{r} \ldots a_{n} \Rightarrow_{\mathcal{G}} a_{1} \ldots a_{i} y_{1} \ldots y_{k} a_{r} \ldots a_{n}$ be the last rewriting step. By induction hypothesis, since $s \Rightarrow_{\mathcal{G}}^{*} a_{1} \ldots a_{i} x_{1} \ldots x_{m} a_{r} \ldots a_{n}$ in fewer rewriting steps, we have $\Phi_{\mathcal{G}}, a_{1}, \ldots, a_{i}, x_{1}, \ldots, x_{m}, a_{r}, \ldots, a_{n}$. Since $x_{1} \ldots x_{m} \Rightarrow y_{1} \ldots y_{k}$ is a rule of $\mathcal{G}$, we have $\left(x_{1} \cdot \ldots \cdot x_{m}\right) /\left(y_{1} \cdot \ldots \cdot y_{m}\right) \in \mathcal{A}_{\mathcal{G}}$. Now the needed sequent $\Phi_{\mathcal{G}}, a_{1}, \ldots, a_{i}, y_{1}, \ldots, y_{m}, a_{r}, \ldots, a_{n} \rightarrow s$ is derived as follows, using the 'landing' rule provided by Definition 5 item 2

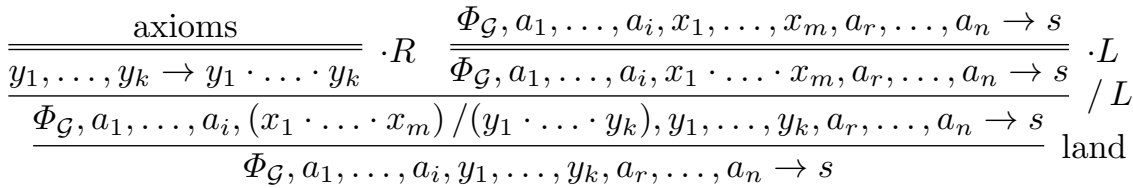

(Here and further double horizontal line means several applications of the rule.)

$2 \Rightarrow 3$ is obvious, since one just takes the trivial bracketing $\Delta=a_{1}, \ldots, a_{n}$.

$3 \Rightarrow 4$ Since $a_{1}, \ldots, a_{n}=\pi_{q}(\Delta)$ and $\mathcal{L}$ is $\pi_{q}$-sound in $! \mathbf{L}^{*}$, the sequent $\pi_{q}\left(\Phi_{\mathcal{G}}\right), a_{1}, \ldots, a_{n} \rightarrow s$ is derivable in $\mathbf{~ L}^{*}$. Next, proceed as follows, using 
item 3 of Definition 5 ,

$$
\frac{! B_{1}, \ldots, ! B_{N} \rightarrow \prod \pi_{q}\left(\Phi_{\mathcal{G}}\right) \quad \frac{\pi_{q}\left(\Phi_{\mathcal{G}}\right), a_{1}, \ldots, a_{n} \rightarrow s}{\overline{\prod \pi_{q}\left(\Phi_{\mathcal{G}}\right), a_{1}, \ldots, a_{n} \rightarrow s}} \cdot L}{! B_{1}, \ldots, ! B_{N}, a_{1}, \ldots, a_{n} \rightarrow s} \text { cut }
$$

$4 \Rightarrow 1$ This part comes directly from the standard undecidability proof for $\mathbf{L}^{*}$, see Kanovich et al. (2019a). Consider the derivation of the sequent $! A_{1}, \ldots, ! A_{N}, a_{1}, \ldots, a_{n} \rightarrow s$ in $! \mathbf{L}^{*}$. The cut rule in $! \mathbf{L}^{*}$ is eliminable (Kanovich et al. 2019a), so we can suppose that this derivation is cut-free. All formulae in this derivation are subformulae of the goal sequent, and the only applicable rules are $\cdot L, \cdot R, / L$, and rules operating ! in the antecedent: $! L, ! C_{1,2}, ! W$.

Now let us hide all the formulae which include / or !. This trivialises all !-operating rules. Next, let us replace all 's in the antecedents with commas. This, in its turn, trivialises $\cdot L$. All sequents in our derivation are now of the form $b_{1}, \ldots, b_{\ell} \Rightarrow C$, where $\ell \geq 0$ and $C=c_{1} \cdot \ldots \cdot c_{r}(r \geq 1)$ or $C=\mathbf{1}$. For the sake of uniformity, we also write $C=\mathbf{1}$ as $C=c_{1} \cdot \ldots \cdot c_{r}$ with $r=0$. The / $L$ rule reduces to

$$
\frac{b_{i+1}, \ldots, b_{j} \rightarrow y_{1} \cdot \ldots \cdot y_{k} \quad b_{1}, \ldots, b_{i}, x_{1}, \ldots, x_{m}, b_{j+1}, \ldots, b_{s} \rightarrow C}{b_{1}, \ldots, b_{i}, b_{i+1}, \ldots, b_{j}, b_{j+1}, \ldots, b_{s} \rightarrow C}
$$

where $x_{1} \ldots x_{m} \Rightarrow y_{1} \ldots y_{k}$ is a rewriting rule of $\mathcal{G}$. For each $\varepsilon$-rule $x_{1} \ldots x_{m} \Rightarrow$ $\varepsilon$ in $\mathcal{G}$ we get the rule

$$
\frac{b_{1}, \ldots, b_{i}, x_{1}, \ldots, x_{m}, b_{i+1}, \ldots, b_{s} \rightarrow C}{b_{1}, \ldots, b_{i}, b_{i+1}, \ldots, b_{s} \rightarrow C}
$$

(which is the reduction of $! L$ for $!\left(x_{1} \cdot \ldots \cdot x_{m}\right)$ ). Finally, $\cdot R$ transforms into

$$
\frac{b_{1}, \ldots, b_{i} \rightarrow c_{1} \cdot \ldots \cdot c_{j} \quad b_{i+1}, \ldots, b_{\ell} \rightarrow c_{j+1} \cdot \ldots \cdot c_{r}}{b_{1}, \ldots, b_{i}, b_{i+1}, \ldots, b_{\ell} \rightarrow c_{1} \cdot \ldots \cdot c_{j} \cdot c_{j+1} \cdot \ldots \cdot c_{r}}
$$

and axioms are of the form $a \rightarrow a$.

Now straightforward induction on derivation establishes the following fact: if $b_{1}, \ldots, b_{\ell} \rightarrow c_{1} \cdot \ldots \cdot c_{r}$ is derivable in the simplified calculus presented above, then $b_{1} \ldots b_{\ell}$ is derivable from $c_{1} \ldots c_{r}$ in the type- 0 grammar $\mid G c$. This finishes our proof.

Theorem 4 and Lemma 1 immediately yield the following generic undecidability result ("meta-theorem").

Theorem 5 Let $\mathcal{L}$ be $\pi_{q}$-sound in $! \mathbf{L}^{*}$, admit the $\cdot L, \cdot R$, and $/ L$ rules, and internalise finite sets of Lambek formulae. Then the derivability problem in $\mathcal{L}$ is undecidable. 
Now, in order to prove undecidability, it is sufficient to show that the calculi considered in this paper internalise finite sets of Lambek formulae. The easiest example is $! \mathbf{L}^{*}$, the multiplicative-additive Lambek calculus with a full-power exponential modality. A set $\mathcal{A}=\left\{A_{1}, \ldots, A_{N}\right\}$ is internalised in ! $\mathbf{L}^{*}$ by $\Phi=! A_{1}, \ldots, ! A_{N}$ (cf. Proposition 6). For $!^{\mathrm{r}} \mathbf{L}^{*}$, the situation is a bit trickier, since in the absence of the weakening rule $! A_{1}, \ldots, ! A_{N}, s \rightarrow s$ (item 1 of Definition 5 is not derivable. This issue is handled by extending $\Phi$ with extra formulae which neutralise $! A_{i}$. Namely, $\Phi=\mathbf{1} / ! A_{1}, ! A_{1}, \ldots \mathbf{1} / ! A_{N}, ! A_{N}$ internalises $\mathcal{A}$ in $\mathbf{~}^{\mathrm{r}} \mathbf{L}^{*}$ (Kanovich et al. 2019a). Actually, the $\mathbf{1}$ constant here can be replaced by $s / s$.

For the calculi with brackets, which interact with the contraction rule, we go further along this line. We still have to add formulae like $\mathbf{1} / ! A_{i}$, for item 1 of Definition 5. but now we also need to neutralise the changes which the contraction rule makes on the bracketing structures. We carry this strategy out in Propositions 7 and 8 below.

We start with systems without stoups: $!_{\mathbf{b}}^{\mathbf{2 0 1 5}} \mathbf{L}^{*} \mathbf{b}, !_{\mathbf{b}}^{\mathbf{2 0 1 8}} \mathbf{L}^{*} \mathbf{b}$, and $!_{\mathbf{b}}^{\mathbf{2 0 1 8}} \mathbf{L b}$.

Proposition 7 The meta-formula

$$
\Phi=!\left((s / s) / ![]^{-1} A_{1}\right), ![]^{-1} A_{1}, \ldots, !\left((s / s) / ![]^{-1} A_{N}\right), ![]^{-1} A_{N}
$$

internalises $\left\{A_{1}, \ldots, A_{N}\right\}$ in $!_{\mathbf{b}}^{\mathbf{2 0 1 5}} \mathbf{L}^{*} \mathbf{b}$.

Proof 1 . The sequent $\Phi, s \rightarrow s$ is derived in $!_{\mathbf{b}}^{\mathbf{2 0 1 5}} \mathbf{L}^{*} \mathbf{b}$ as follows:

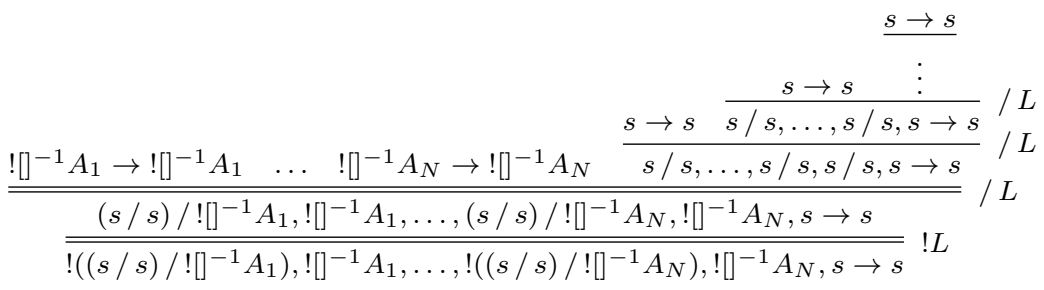

2. The 'landing' rule is derived in $!_{\mathbf{b}}^{\mathbf{2 0 1 5}} \mathbf{L}^{*} \mathbf{b}$ as follows:

$$
\begin{aligned}
& \frac{\Phi, \Delta_{1}, A_{i}, \Delta_{2} \rightarrow C}{\Phi, \Delta_{1},\left[[]^{-1} A_{i}\right], \Delta_{2} \rightarrow C}[]^{-1} L \\
& \frac{\Phi, \Delta_{1},\left[![]^{-1} A_{i}\right], \Delta_{2} \rightarrow C}{\Phi, \Delta_{1}, \Delta_{2} \rightarrow C} ! L \\
& ! C \text { applied to } ![]^{-1} A_{i}
\end{aligned}
$$

3. Notice that

$$
\pi_{q}(\Phi)=!\left((s / s) / ! A_{1}\right), ! A_{1}, \ldots, !\left((s / s) / ! A_{N}\right), ! A_{N} .
$$

Next, by applying $\cdot R$ to $\Lambda \rightarrow !\left((s / s) / ! A_{1}\right) ; ! A_{1} \rightarrow ! A_{1} ; \ldots \Lambda \rightarrow !\left((s / s) / ! A_{N}\right)$; $! A_{1} \rightarrow ! A_{N}$, we get the necessary sequent $! B_{1}, \ldots, ! B_{N} \rightarrow \prod \pi_{q}(\Phi)$.

The sequents $\Lambda \rightarrow !\left((s / s) / ! A_{i}\right)$ are derived in $! \mathbf{L}^{*}$ as follows: 


$$
\frac{\frac{s \rightarrow s}{\Lambda \rightarrow s / s}}{\frac{\frac{1 A_{1} \rightarrow s / s}{\Lambda \rightarrow(s / s) / ! A_{1}}}{\Lambda \rightarrow !\left((s / s) / ! B_{1}\right)}}
$$

Proposition 8 The meta-formula

$$
\Phi=!\left((s / s) / ! Z_{1}\right), ! Z_{1}, \ldots, !\left((s / s) / ! Z_{n}\right), ! Z_{n}, !((s / s) /\langle\rangle\langle\rangle q),[[q]],
$$

where

$$
Z_{i}=\left([]^{-1}\left(! A_{i} \cdot\langle\rangle\langle\rangle q\right)\right) / q,
$$

internalises $\left\{A_{1}, \ldots, A_{n}\right\}$ in $!_{\mathbf{b}}^{\mathbf{2 0 1 8}} \mathbf{L}^{*} \mathbf{b}$ and $!_{\mathbf{b}}^{\mathbf{2 0 1 8}} \mathbf{L b}$.

Notice that in $!_{\mathbf{b}}^{\mathbf{2 0 1 8}} \mathbf{L b}$ we do not need to impose any additional nonemptiness restriction on the 'landing' rule, since Lambek's restriction is automatically satisfied by non-emptiness of $\Phi$. Thus, for our undecidability proof $!_{\mathrm{b}}^{2018} \mathbf{L b}$ is capable of encoding arbitrary semi-Thue systems, even the ones which include $\varepsilon$-rules.

For categorial grammars, however, Lambek's restriction will make a difference in the expressive power of $!_{\mathbf{b}}^{2018} \mathbf{L b}$ as opposed to $!_{\mathrm{b}}^{\mathbf{2 0 1 8}} \mathbf{L}^{*} \mathbf{b}$, as explained in the next section.

Proof 1 . The sequent $\Phi, s \rightarrow s$ is derived in $!_{\mathbf{b}}^{\mathbf{2 0 1 8}} \mathbf{L b}$ (and therefore in $\mathbf{!}_{\mathbf{b}}^{\mathbf{2 0 1 8}} \mathbf{L}^{*} \mathbf{b}$ ) as follows:

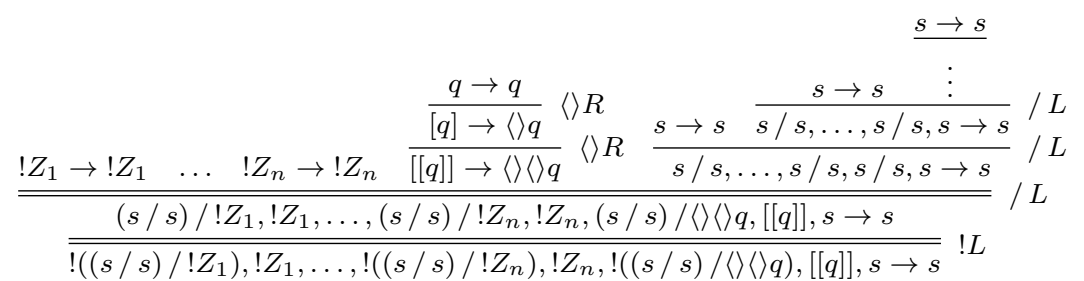

Notice that all antecedents in this derivation are non-empty, so Lambek's restriction is observed.

2. Let $\Phi^{\prime}$ be $\left.(s / s) / ! Z_{1}\right), ! Z_{1}, \ldots, !\left((s / s) / ! Z_{n}\right), ! Z_{n}, !((s / s) /\langle\rangle\langle\rangle q)$, that is, $\Phi=\Phi^{\prime},[[q]]$, and recall that $! Z_{i}=!\left(\left([]^{-1}\left(! A_{i} \cdot\langle\rangle\langle\rangle q\right)\right) / q\right)$. Now the 'landing' rule is derived in $!_{\mathbf{b}}^{2018} \mathbf{L b}$ (and therefore also in $!_{\mathbf{b}}^{2018} \mathbf{L} * \mathbf{b}$ ) as follows.

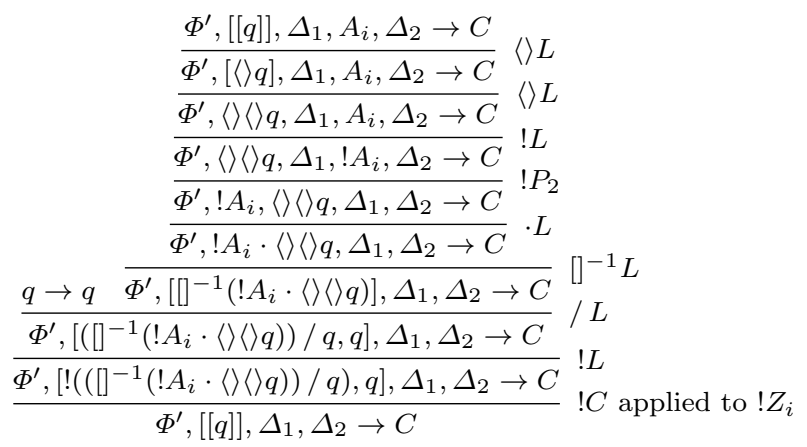


Again, notice how Lambek's restriction is maintained by non-emptiness of $\Phi^{\prime}$ and by the $q$ in the brackets (when applying $! C$ ).

3. Notice that

$$
\begin{aligned}
\pi_{q}(\Phi)=!\left((s / s) / !\left(! A_{1} \cdot \mathbf{1}\right)\right) / \mathbf{1}, !\left(! A_{1} \cdot \mathbf{1}\right), \ldots, \\
!\left((s / s) / !\left(! A_{n} \cdot \mathbf{1}\right)\right) / \mathbf{1}, !\left(! A_{n} \cdot \mathbf{1}\right), !((s / s) / \mathbf{1}), \mathbf{1} .
\end{aligned}
$$

Next, we enjoy the following derivations in $! \mathbf{L}^{*}$ :

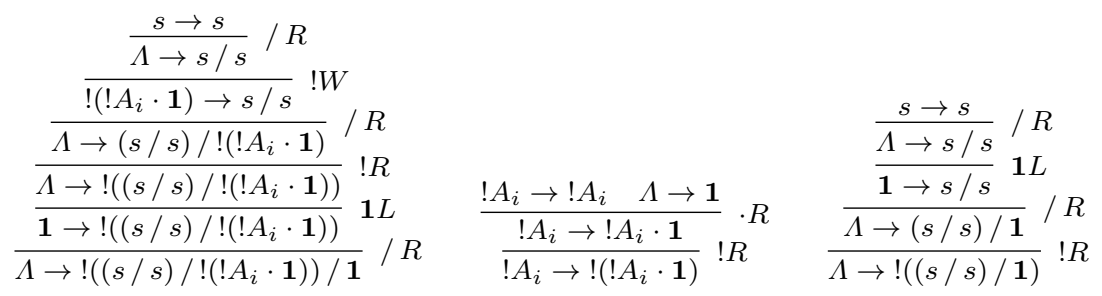

Also recall that $\Lambda \vdash \mathbf{1}$ is an axiom of ! $\mathbf{L}^{*}$. Now several applications of $\cdot L$ yield $! A_{1}, \ldots, ! A_{n} \rightarrow \prod \pi_{q}(\Phi)$.

Now by Theorem 5 we get undecidability for the systems without stoups.

Theorem 6 The derivability problems for $!_{\mathbf{b}}^{\mathbf{2 0 1 5}} \mathbf{L}^{*} \mathbf{b}, !_{\mathbf{b}}^{\mathbf{2 0 1 8}} \mathbf{L}^{*} \mathbf{b}$, and $!_{\mathbf{b}}^{\mathbf{2 0 1 8}} \mathbf{L b}$ are undecidable.

For systems with stoups, the situation is as follows. First, concerning derivability of sequents with empty stoups, $!_{\mathrm{b}}^{\mathbf{2 0 1 5}} \mathbf{L}^{*} \mathbf{b}(\mathrm{st})^{\prime}, !_{\mathrm{b}}^{\mathbf{2 0 1 8}} \mathbf{L}^{*} \mathbf{b}(\mathrm{st})^{\prime}$, and $!_{\mathrm{b}}^{2018} \mathbf{L b}(\mathrm{st})^{\prime}$ just equivalent to $!_{\mathrm{b}}^{\mathbf{2 0 1 5}} \mathbf{L}^{*} \mathrm{~b}, !_{\mathrm{b}}^{\mathbf{2 0 1 8}} \mathbf{L}^{*} \mathrm{~b}$, and $!_{\mathrm{b}}^{\mathbf{2 0 1 8}} \mathbf{L b}$ respectively (Propositions 1 and 2). This gives undecidability for these systems.

Theorem 7 The derivability problems for $!_{\mathbf{b}}^{\mathbf{2 0 1 5}} \mathbf{L}^{*} \mathbf{b}(\mathrm{st})^{\prime}, !_{\mathbf{b}}^{\mathbf{2 0 1 8}} \mathbf{L}^{*} \mathbf{b}(\mathrm{st})^{\prime}$, and $!_{\mathbf{b}}^{\mathbf{2 0 1 8}} \mathbf{L b}(\mathrm{st})^{\prime}$ are undecidable.

Proving undecidability for original Morrill's systems, $!_{\mathbf{b}}^{\mathbf{2 0 1 5}} \mathbf{L}^{*} \mathbf{b}(\mathrm{st})$ and $!_{\mathrm{b}}^{\mathbf{2 0 1 8}} \mathbf{L}^{*} \mathbf{b}$ (st) (which we altered in order to gain cut elimination, see Section 4), requires some extra work. For $!_{b}^{\mathbf{2 0 1 5}} \mathbf{L}^{*} \mathbf{b}(\mathrm{st})$ it is easy. The right rule for ! is never used in the proof of item 1 in Proposition 7. Also, in the landing rule (item 2) there are no other !-formulae moved into the newly created bracketed island. Thus, from the point of view of Proposition $7 !_{\mathbf{b}}^{\mathbf{2 0 1 5}} \mathbf{L}^{*} \mathbf{b}(\mathrm{st})$ is indistinguishable from $!_{\mathbf{b}}^{\mathbf{2 0 1 5}} \mathbf{L}^{*} \mathbf{b}(\mathrm{st})^{\prime}$, and this yields the necessary undecidability result.

Theorem 8 The derivability problem for $!_{\mathbf{b}}^{\mathbf{2 0 1 5}} \mathbf{L}^{*} \mathbf{b}(\mathrm{st})$ is undecidable.

The case of $!_{\mathbf{b}}^{\mathbf{2 0 1 8}} \mathbf{L} * \mathbf{b}($ st) is trickier. The issue is that the $! L$ rule in this calculus is not invertible, i.e., derivability of $\Xi\left(\zeta ; \Delta_{1}, ! A, \Delta_{2}\right) \rightarrow C$ is not always equivalent to derivability of $\Xi\left(\zeta, A ; \Delta_{1}, \Delta_{2}\right) \rightarrow C$. In particular, ! $p \rightarrow$ ! $p$ is derivable, while $p ; \Lambda \rightarrow ! p$ is not. For our construction this issue is crucial. Namely, when proving the 'landing' rule (item 2 of Definition 5), in 
$!_{\mathbf{b}}^{\mathbf{2 0 1 8}} \mathbf{L}^{*} \mathbf{b}$ (st) we would have to move the !-formulae from $\Phi$ to the stoup. Otherwise, we could not operate the contraction rule. This, however, would cause problems with item 1 the left premises of the derivation (see proof of Proposition 80 become $Z_{i} ; \Lambda \rightarrow ! Z_{i}$ (instead of $! Z_{i} \rightarrow ! Z_{i}$ ), and in general are not derivable.

Fortunately, this issue is easily resolved by adding an extra! on $Z_{i}$ (Kanovich et al. 2019b). Indeed, $! Z_{i} ; \Lambda \rightarrow ! Z_{i}$ is derivable from $! Z_{i} \rightarrow ! Z_{i}$ by application of $! P$. This yields the following internalisation property, where we essentially use the stoup for !-formulae in $\Phi$ (the rightmost [[q]] is kept outside the stoup).

Proposition 9 The meta-formula

$$
\Phi=(s / s) / ! Z_{1}, ! Z_{1}, \ldots,(s / s) / ! Z_{N}, ! Z_{N},(s / s) /\langle\rangle\langle\rangle q ;[[q]],
$$

where

$$
Z_{i}=\left([]^{-1}\left(! A_{i} \cdot\langle\rangle\langle\rangle q\right)\right) / q
$$

satisfies items 1 and 2 of Definition 5 for internalisation of $\left\{A_{1}, \ldots, A_{N}\right\}$ in $!_{\mathbf{b}}^{\mathbf{2 0 1 8}} \mathbf{L}^{*} \mathbf{b}(\mathrm{st})$.

Proof 1 . The sequent $\Phi, s \rightarrow s$ is derived in $!_{\mathbf{b}}^{\mathbf{2 0 1 8}} \mathbf{L} * \mathbf{b}$ (st) as follows:

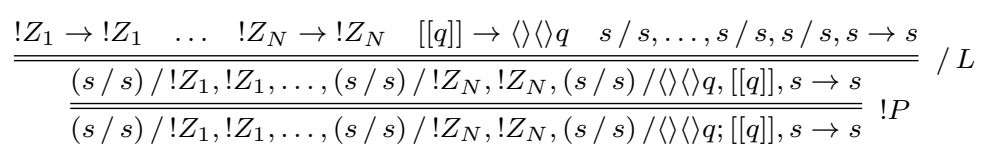

2. Let $\zeta$ be the stoup of $\Phi$, that is, $\Phi=\zeta ;[[q]]$. Then the "landing" rule is established as follows:

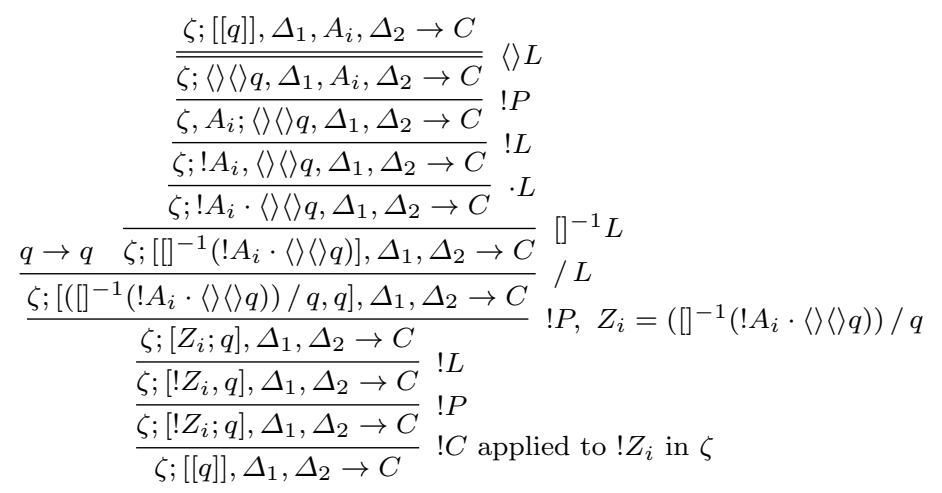

Now we can finalise our undecidability proof for $!_{\mathbf{b}}^{2018} \mathbf{L}^{*} \mathbf{b}(\mathrm{st})$.

Lemma 2 Let $\mathcal{A}_{\mathcal{G}}=\left\{A_{1}, \ldots, A_{N}\right\}$ and let $Z_{i}=\left([]^{-1}\left(! A_{i} \cdot\langle\rangle\langle\rangle q\right)\right) / q(i=$ $1, \ldots, N)$. Then the sequent

$!\left((s / s) / ! Z_{1}\right), ! ! Z_{1}, \ldots, !\left((s / s) / ! Z_{n}\right), ! ! Z_{n}, !((s / s) /\langle\rangle\langle\rangle q),[[q]], a_{1}, \ldots, a_{n} \rightarrow s$ is derivable in $!_{\mathbf{b}}^{\mathbf{2 0 1 8}} \mathbf{L}^{*} \mathbf{b}(\mathrm{st})$ if and only if $s \Rightarrow_{\mathcal{G}}^{*} a_{1} \ldots a_{n}$. 
Proof For the "if" direction, we use Proposition 9 and proceed exactly as in the $1 \Rightarrow 2$ implication from the proof of Lemma 1. This gives derivability of

$$
(s / s) / ! Z_{1}, ! Z_{1}, \ldots,(s / s) / ! Z_{n}, ! Z_{n},(s / s) /\langle\rangle\langle\rangle q ;[[q]], a_{1}, \ldots, a_{n} \rightarrow s,
$$

which yields the necessary sequent

$!\left((s / s) / ! Z_{1}\right), ! ! Z_{1}, \ldots, !\left((s / s) / ! Z_{n}\right), ! ! Z_{n}, !((s / s) /\langle\rangle\langle\rangle q),[[q]], a_{1}, \ldots, a_{n} \rightarrow s$

by several applications of $! L$.

For the "only if" direction, since the sequent in question is derivable in $!_{\mathrm{b}}^{\mathbf{2 0 1 8}} \mathbf{L}^{*} \mathbf{b}$ (st), it is also derivable in $!_{\mathrm{b}}^{\mathbf{2 0 1 8}} \mathbf{L}^{*} \mathbf{b}$ (Proposition 3). By cut with $! Z_{i} \rightarrow ! ! Z_{i}$ (which is derivable in $!_{\mathbf{b}}^{\mathbf{2 0 1 8}} \mathbf{M A L C} \mathbf{C}^{*} \mathbf{b}$ ) we get derivability of

$!\left((s / s) / ! Z_{1}\right), ! Z_{1}, \ldots, !\left((s / s) / ! Z_{n}\right), ! Z_{n}, !((s / s) /\langle\rangle\langle\rangle q),[[q]], a_{1}, \ldots, a_{n} \rightarrow s$.

Recall that cut is admissible in $!_{\mathbf{b}}^{\mathbf{2 0 1 8}} \mathbf{L}^{*} \mathbf{b}$ by Proposition 1. Now we use Proposition 8 and the $4 \Rightarrow 1$ implication of Lemma 1 and conclude that $s \Rightarrow_{\mathcal{G}}^{*} a_{1} \ldots a_{n}$.

Theorem 9 The derivability problem in $!_{\mathbf{b}}^{\mathbf{2 0 1 8}} \mathbf{L}^{*} \mathbf{b}(\mathrm{st})$ is undecidable.

Proof Immediately by Lemma 2 and Theorem 4

\section{Generative Power of Categorial Grammars}

Besides undecidability results, Lemma1 has another corollary: categorial grammars based on the calculi with subexponentials considered throughout this paper generate exactly the class of all recursively enumerable languages.

The definitions of categorial grammars are given in Section 2 for calculi without brackets and in Section 3 for bracketed systems. Recall that for the latter we distinguish two notions of recognition, namely s-recognition and trecognition.

Let us briefly survey known results characterising classes of languages generated by categorial grammars over different extensions of the Lambek calculus.

For the pure (multiplicative-only) Lambek calculus this class coincides with the class of context-free languages. The hard part, Lambek to context-free, was done by Pentus (1993). For the easier direction, context-free to Lambek, we refer to Gaifman (Bar-Hillel et al. 1960), as the first one who obtained the result, and Buszkowski (1985), for a modern way of proving it using Greibach normal form (Greibach, 1965) 1

Adding the unit constant, $\mathbf{1}$, does not extend the class of languages generated by Lambek grammars - all $\mathbf{L}_{\mathbf{1}}$-languages are still context-free (Kuznetsov $2012 \mathrm{~b}$ ).

\footnotetext{
1 Methods of Gaifman and Buszkowski work only for context-free languages without the empty word. The empty word case was handled by Kuznetsov (2012a).
} 
Grammars based on the Lambek calculus with brackets, $\mathbf{L} \mathbf{b}^{*}$, also generate only context-free languages, both in the sense of s- and t-recognition. This was initially claimed by Jäger (2003), but, as noticed by Fadda and Morrill (2005), his proof for t-recognition relied on an incorrect lemma by Versmissen (1996). A correct proof was given by Kanazawa (2018).

Additive connectives, $\vee$ and $\wedge$, increase the generative power of Lambek grammars. Namely, as noticed by Kanazawa (1992), MALC*-grammars an generate finite intersections of context-free languages and, moreover, images of such intersections under symbol-to-symbol homomorphisms (that is, homomorphisms $h: \Sigma^{*} \rightarrow \Sigma^{*}$ that map $\Sigma$ to $\left.\Sigma\right)$. Furthermore, as shown by Kuznetsov (2013) and Kuznetsov and Okhotin (2017), the class of MALC* languages includes the class of languages generated by conjunctive grammars Okhotin (2013). This latter class is strictly greater than the class of intersections of context-free languages, but, unless $\mathrm{P}=\mathrm{NP}$, is still not closed under symbol-to-symbol homomorphisms (Kuznetsov and Okhotin, 2017).

In this section we show that adding the (sub)exponential modality, even constrained by brackets, increases the power of Lambek categorial grammars to the highest possible level - all recursively enumerable (r.e.) languages.

From many definitions of the class of r.e. languages we choose the one based on type- 0 grammars (see Section 9 above for definition). A language $M$ over alphabet $\Sigma$ is r.e. if and only if there exists a type-0 grammar $\mathcal{G}$ such that $M=\left\{w \in \Sigma^{*} \mid s \Rightarrow_{\mathcal{G}}^{*} w\right\}$, i.e., $M$ is the language generated by $\mathcal{G}$.

Obviously, all languages generated by categorial grammars based on calculi considered in this paper are r.e. The converse statement is non-trivial, and extends undecidability results of the previous section. For bracketed calculi, moreover, we have two notions of recognition (s-recognition and t-recognition).

As in the previous section, we provide a generic result. Notice how Lambek's restriction comes into play here.

Theorem 10 1. Let $\mathcal{L}$ satisfy the conditions of Theorem 5 and additionally admit \langle\rangle $L$ and $\backslash R$ without Lambek's restriction. Then any r.e. language can be generated by a categorial grammar based on $\mathcal{L}$.

2. Let $\mathcal{L}$ satisfy the conditions of Theorem 5 and admit \langle\rangle $L$ and $\backslash R$ with Lambek's restriction. Then any r.e. language without the empty word can be generated by a categorial grammar based on $\mathcal{L}$.

Proof Consider the type-0 grammar $\mathcal{G}$ which generates the given r.e. language. Let $\Phi_{\mathcal{G}}$ internalise $\mathcal{G}$ in $\mathcal{L}$. We are going to prove that the following three statements are equivalent:

1. a word $a_{1} \ldots a_{n}$ belongs to the language generated by $\mathcal{G}$ (that is, $s \Rightarrow_{\mathcal{G}}^{*}$ $\left.a_{1} \ldots a_{n}\right)$;

2. the sequent $a_{1}, \ldots, a_{n} \rightarrow \prod \Phi_{\mathcal{G}} \backslash s$ is derivable in $\mathcal{L}$;

3. the sequent $\Delta \rightarrow \prod \Phi_{\mathcal{G}} \backslash s$ is derivable in $\mathcal{L}$ for some bracketing $\Delta$ of $a_{1}, \ldots, a_{n}$.

The meta-formula $\Phi_{\mathcal{G}}$ could contain brackets; for brackets, we define $\prod$ as follows: $\Pi[\Gamma]=\langle\rangle\left(\prod \Gamma\right)$. 
In order to establish $1 \Rightarrow 2$, apply Lemma 1 Let $s \Rightarrow_{\mathcal{G}}^{*} a_{1} \ldots a_{n}$. We get $\Phi_{\mathcal{G}}, a_{1}, \ldots, a_{n} \rightarrow s$ derivable in $\mathcal{L}$. Applying $\cdot L$ and \langle\rangle $L$ several times, we obtain $\prod \Phi_{\mathcal{G}}, a_{1}, \ldots, a_{n} \rightarrow s$. Now we apply $/ R$. In Case 1 , without Lambek's restriction, we always get $a_{1}, \ldots, a_{n} \rightarrow \prod \Phi_{\mathcal{G}} \backslash s$. In Case 2 , this is possible only for $n>0$, and in this case we consider only type- 0 grammars which do not generate the empty word.

The $2 \Rightarrow 3$ implication is established by taking the trivial bracketing $\Delta=$ $a_{1}, \ldots, a_{n}$. Finally, for the backwards $3 \Rightarrow 1$ implication we use $\pi_{q^{\text {-soundness }}}$ of $\mathcal{L}$ in ! $\mathbf{L}^{*}$ and derive $a_{1}, \ldots, a_{n} \rightarrow \prod \pi_{q}\left(\Phi_{\mathcal{G}}\right) \backslash s$ in $! \mathbf{L}^{*}$.

In $\mathbf{~ L}^{*}$, the $\backslash R$ rule is invertible using cut:

$$
\frac{\Pi \rightarrow F \backslash E \quad \frac{F \rightarrow F \quad E \rightarrow E}{F, F \backslash E \rightarrow E} \backslash L}{F, \Pi \rightarrow E} \text { cut }
$$

Thus, we get $\prod \pi_{q}\left(\Phi_{\mathcal{G}}\right), a_{1}, \ldots, a_{n} \rightarrow s$, and by cut with item 3 of Definition 5 we get $! A_{1}, \ldots, ! A_{N}, a_{1}, \ldots, a_{n} \rightarrow s$. The $4 \Rightarrow 1$ implication of Lemma 1 finishes the job.

Now the necessary categorial grammar is constructed as follows. The lexicon $\triangleright$ is trivial, just the identity relation: $\triangleright=\{\langle a, a\rangle \mid a \in \Sigma\}$. All the information is kept in the goal formula $H=\prod \pi_{q}\left(\Phi_{\mathcal{G}}\right) \backslash s$. By definition, this grammar generates the same language as $\mathcal{G}$, both in the sense of s-recognition and t-recognition.

Notice that the grammars constructed in Theorem 10 have the property of unique type assignment: for each letter $a$ there exists exactly one formula $A$ such that $a \triangleright A$. For the pure Lambek calculus, constructing such grammars is much harder. However, for each context-free language without the empty word there exists a Lambek grammar with unique type assignment, as shown by Safiullin (2007), see also Kuznetsov (2017).

Internalisation properties (Propositions 7, 8, 9) now yield the following theorems.

Theorem 11 Let $M$ be a recursively enumerable language. Then

1. there exists a ! $\mathbf{L}^{*}$-grammar which generates $M$;

2. there exists a $!^{\mathbf{r}} \mathbf{L}^{*}$-grammar which generates $M$;

3. for each of the calculi $!_{\mathbf{b}}^{\mathbf{2 0 1 5}} \mathbf{L}^{*} \mathbf{b}(\mathrm{st}), !_{\mathbf{b}}^{\mathbf{2 0 1 5}} \mathbf{L}^{*} \mathbf{b}(\mathrm{st})^{\prime}, !_{\mathbf{b}}^{\mathbf{2 0 1 5}} \mathbf{L}^{*} \mathbf{b}, !_{\mathbf{b}}^{\mathbf{2 0 1 8}} \mathbf{L}^{*} \mathbf{b}(\mathrm{st})$, $!_{\mathbf{b}}^{\mathbf{2 0 1 8}} \mathbf{L}^{*} \mathbf{b}(\mathrm{st})^{\prime}, !_{\mathbf{b}}^{\mathbf{2 0 1 8}} \mathbf{L}^{*} \mathbf{b}$ there exist a grammar which generates $M$, both in the sense of s-recognition and t-recognition.

Theorem 12 Let $M$ be a recursively enumerable language without the empty word. Then for each of the calculi $!_{\mathbf{b}}^{\mathbf{2 0 1 8}} \mathbf{L b}(\mathrm{st})^{\prime}$ and $!_{\mathbf{b}}^{\mathbf{2 0 1 8}} \mathbf{L b}$ there exists a grammar which generates $M$, both in the sense of s-recognition and t-recognition. 


\section{Undecidability for One-Division Fragments}

In this section we strengthen our complexity lower bounds by restricting ourselves to the smallest non-trivial fragment of the Lambek calculus with only one division operation, extended by ! and, in the bracketed case, bracket modalities \langle\rangle and []$^{-1}$. For these one-division systems, we obtain the same complexity results. Thus, the situation is different from the pure Lambek: while checking derivability in the Lambek calculus (without additives, brackets, and exponentials) is an NP-complete problem (Pentus, 2006), for the one-division fragment there exists a polynomial time algorithm (Savateev, 2010 Kuznetsov, 2016). In contrast, in the presence of ! the one-division fragment is as powerful, as the whole system, both with and without brackets.

Our construction is based on Buszkowski's method of encoding type-0 grammars in the one-division fragment of the Lambek calculus extended with extra (non-logical) axioms (Buszkowski, 1982). Then these non-logical axioms are internalised using !, in the same way as in Section 9. We present a new, simplified version of Buszkowski's construction. This version does not require the type-0 grammar to be translated into binary normal form, and, in particular, works also for languages with the empty word.

Recall that the one-division fragment of the Lambek calculus uses formulae constructed from variables using only one division operation, /. We denote this fragment by $\mathbf{L}^{*}(/)$ (the asterisk means that we do not impose Lambek's restriction). Axioms of $\mathbf{L}^{*}(/)$ are of the form $A \rightarrow A$, and its rules of inference are as follows:

$$
\begin{gathered}
\frac{\Pi \rightarrow A \quad \Delta_{1}, B, \Delta_{2} \rightarrow C}{\Delta_{1}, B / A, \Pi, \Delta_{2} \rightarrow C} / L \quad \frac{\Pi, A \rightarrow B}{\Pi \rightarrow B / A} / R \\
\frac{\Pi \rightarrow A \quad \Delta_{1}, A, \Delta_{2} \rightarrow C}{\Delta_{1}, \Pi, \Delta_{2} \rightarrow C} \text { cut }
\end{gathered}
$$

Let us call a B-rule (after Buszkowski) an inference rule of the following form:

$$
\frac{\Delta, q_{1}, \ldots, q_{m} \rightarrow r}{p_{1}, \ldots, p_{k}, \Delta \rightarrow t} B_{q_{1}, \ldots, q_{m}, r ; p_{1}, \ldots, p_{k}, t}
$$

In this rule, $q_{1}, \ldots, q_{m}, p_{1}, \ldots, p_{k}, r$, and $t$ are concrete variables from Var, not meta-variables. Each tuple of variables produces an independent B-rule. On the other hand, $\Delta$ stands for an arbitrary sequence of Lambek formulae (in the one-division language).

With each rule, we associate the following Gentzen-style rule, which we call a $\mathrm{B}^{\prime}$-rule:

$$
\frac{\Pi_{1} \rightarrow p_{1} \quad \ldots \quad \Pi_{k} \rightarrow p_{k} \quad \Delta, q_{1}, \ldots, q_{m} \rightarrow r}{\Pi_{1}, \ldots, \Pi_{k}, \Delta \rightarrow t} B_{q_{1}, \ldots, q_{m}, r ; p_{1}, \ldots, p_{k}, t}^{\prime}
$$

and the following $B$-formula $\left.\right|^{2}$

$$
B=\left(t /\left(r / q_{1} \ldots q_{m}\right)\right) / p_{1} \ldots p_{k} .
$$

${ }^{2}$ Here $E / F_{1} \ldots F_{n}$ is used as a shortcut for $\left(E / F_{n}\right) / \ldots / F_{1}$, thus, $B$ is a one-division formula. 
For a B-formula $B$, we introduce the corresponding $B$-axiom $\Lambda \rightarrow B$.

B-rules, B-axioms, and $B^{\prime}$-rules will be used as extensions of the Lambek calculus with one division, and each of the three variants has its benefits:

1. with B-rules, a derivation of a sequent without Lambek connectives (i.e., of the form $z_{1}, \ldots, z_{m} \rightarrow s$ ) is non-branching, which will allow us to encode it using a ! modality with a restricted version of contraction rule;

2. B-formulae are convenient for incorporating into the main sequent using a "deduction theorem" with!;

3. finally, the calculus with $B^{\prime}$-rules, unlike the first two variants, admits cut elimination (see Lemma 4 below), which facilitates analysis of derivations.

B-rules, B-axioms, and B'-rules yield equivalent extensions of the Lambek calculus:

Lemma 3 In the presence of cut, the extensions of $\mathbf{L}^{*}(/)$ with: (1) a set of B-rules; (2) the corresponding set of $\mathrm{B}^{\prime}$-rules; (3) the corresponding set of $B$-axioms are equivalent, i.e., derive the same set of sequents.

Proof $(1) \Rightarrow(2)$ (modelling B-rules using B'-rules)

$$
\frac{p_{1} \rightarrow p_{1} \quad \ldots \quad p_{k} \rightarrow p_{k} \quad \Delta, q_{1}, \ldots, q_{m} \rightarrow r}{p_{1}, \ldots, p_{k}, \Delta \rightarrow t} B_{q_{1}, \ldots, q_{m}, r ; p_{1}, \ldots, p_{k}, t}^{\prime}
$$

$(2) \Rightarrow(3) \quad$ (modelling $\mathrm{B}^{\prime}$-rules using B-axioms and cut)

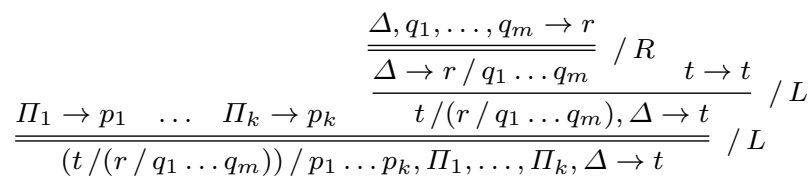

Then a cut with the B-axiom $\Lambda \rightarrow\left(t /\left(r / q_{1} \ldots q_{m}\right)\right) / p_{1} \ldots p_{k}$ yields the goal sequent $\Pi_{1}, \ldots, \Pi_{k}, \Delta \rightarrow t$.

$(3) \Rightarrow(1)$ (deriving B-axioms using B-rules)

$$
\begin{aligned}
& \frac{q_{1} \rightarrow q_{1} \quad \ldots \quad q_{m} \rightarrow q_{m} \quad r \rightarrow r}{\underline{\underline{r / q_{1} \ldots q_{m}, q_{1}, \ldots, q_{m} \rightarrow r}}} / L \\
& \frac{\frac{r / q_{1} \ldots q_{m}, q_{1}, \ldots, q_{m} \rightarrow r}{p_{1}, \ldots, p_{k}, r / q_{1} \ldots q_{m} \rightarrow t}}{p_{1}, \ldots, p_{k} \rightarrow t /\left(r / q_{1} \ldots q_{m}\right)} B_{q_{1}, \ldots, q_{m}, r ; p_{1}, \ldots, p_{k}, t} \\
& \frac{\frac{p_{1}, \ldots, p_{k} \rightarrow t /\left(r / q_{1} \ldots q_{m}\right)}{p_{1}, \ldots, p_{k}}}{\Lambda \rightarrow\left(t /\left(r / q_{1} \ldots q_{m}\right)\right) / p_{1} \ldots p_{k}} / R
\end{aligned}
$$

Lemma 4 The one-division Lambek calculus $\mathbf{L}^{*}(/)$, extended with an arbitrary finite set of $\mathrm{B}^{\prime}$-rules, admits cut elimination.

Proof The proof goes via a standard argument, exactly as for the Lambek calculus itself (Lambek, 1958). The global induction is by the number of cuts in a derivation. Each cut is eliminated by nested induction, where the outer 
parameter is the complexity of the formula being cut, and the inner one is the summary derivation depth of the premises of the cut.

The base case is cut with axiom $(A \rightarrow A)$, which just disappears. For the induction step, one distinguishes principal and non-principal cut premises. A premise is called principal, if the last rule in its derivation introduces the formula being cut. Thus, if the left premise is introduced by a $\mathrm{B}^{\prime}$-rule, it is always principal (since this rule introduces the succedent $t$ ). The key trick, however, is that if the right premise of a cut is introduced by a $\mathrm{B}^{\prime}$-rule, then it is never principal. This is due to the fact that a $\mathrm{B}^{\prime}$-rule introduces nothing to the antecedent: the antecedent of its goal, $\Pi_{1}, \ldots, \Pi_{k}, \Delta$, is composed from antecedents of the premises.

Thus, there are three possible cases.

Case 1: the left premise is non-principal. Cut can be exchanged with the last rule in the left premise derivation, and propagates upward. The inner induction parameter gets smaller, while the outer one is intact. Propagation of cut through non-principal $(\rightarrow /)$ and $(/ \rightarrow)$ is standard and is performed exactly as in the cut elimination proof of Lambek (1958). As for B'-rules, such a rule cannot yield a non-principal left premise of cut.

Case 2: the right premise in non-principal. Cut propagates to the right.

This is how cut gets propagated through a $\mathrm{B}^{\prime}$-rule:

$$
\frac{\Psi \rightarrow A \frac{\Pi_{1} \rightarrow p_{1} \quad \ldots \quad \Pi_{k} \rightarrow p_{k} \quad \Delta^{\prime}, A, \Delta^{\prime \prime}, q_{1}, \ldots, q_{m} \rightarrow t}{\Pi_{1}, \ldots, \Pi_{k}, \Delta^{\prime}, A, \Delta^{\prime \prime} \rightarrow t}}{\Pi_{1}, \ldots, \Pi_{k}, \Delta^{\prime}, \Psi, \Delta^{\prime \prime} \rightarrow t} B^{\prime}
$$

transforms into

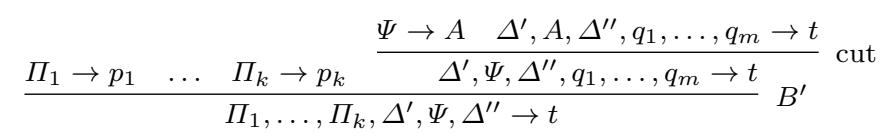

and

$$
\frac{\Psi \rightarrow A \frac{\Pi_{1} \rightarrow p_{1} \quad \ldots \quad \Pi_{i}^{\prime}, A, \Pi_{i}^{\prime \prime} \rightarrow p_{i} \quad \ldots \quad \Pi_{k} \rightarrow p_{k} \quad \Delta, q_{1}, \ldots, q_{m} \rightarrow t}{\Pi_{1}, \ldots, \Pi_{i}^{\prime}, A, \Pi_{i}^{\prime \prime}, \ldots, \Pi_{k}, \Delta \rightarrow t}}{\Pi_{1}, \ldots, \Pi_{i}^{\prime}, \Psi, \Pi_{i}^{\prime \prime}, \ldots, \Pi_{k}, \Delta \rightarrow t} B^{\prime}
$$

transforms into

$$
\frac{\Pi_{1} \rightarrow p_{1} \quad \ldots \quad \frac{\Psi \rightarrow A \quad \Pi_{i}^{\prime}, A, \Pi_{i}^{\prime \prime} \rightarrow p_{i}}{\Pi_{i}^{\prime}, \Psi, \Pi_{i}^{\prime \prime} \rightarrow p_{i}} \text { cut } \quad \ldots \quad \Pi_{k} \rightarrow p_{k} \quad \Delta, q_{1}, \ldots, q_{m} \rightarrow t}{\Pi_{1}, \ldots, \Pi_{i}^{\prime}, \Psi, \Pi_{i}^{\prime \prime}, \ldots, \Pi_{k}, \Delta \rightarrow t} B^{\prime}
$$

Propagation of cut to the right through non-principal / $R$ and / $L$ is again due to Lambek.

Case 3: both left and right premises are principal, being introduced by $/ R$ and / $L$ respectively. In this case cut transforms into two cuts of lower complexity:

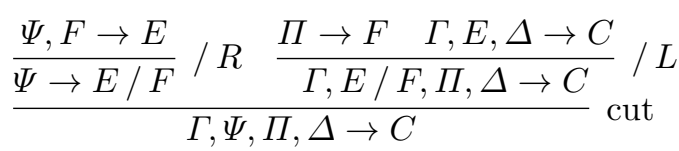


becomes

$$
\frac{\Pi \rightarrow F \quad \frac{\Psi, F \rightarrow E \quad \Gamma, E, \Delta \rightarrow C}{\Gamma, \Psi, F, \Delta \rightarrow C} \mathrm{cut}}{\Gamma, \Psi, \Pi, \Delta \rightarrow C} \mathrm{cut}
$$

(This transformation, again, comes from the original Lambek's proof.)

Now we are ready to present the encoding of type-0 grammars. Consider a grammar $\mathcal{G}=\langle N, \Sigma, P, s\rangle$. For each production $\mathfrak{p}=\left(v_{1} \ldots v_{m} \Rightarrow w_{1} \ldots w_{k}\right) \in$ $P$ add $\mathbf{a}^{\mathfrak{p}}, \mathbf{b}^{\mathfrak{p}}, \mathbf{c}^{\mathfrak{p}}, \mathbf{d}^{\mathfrak{p}}, \mathbf{e}^{\mathfrak{p}}, \mathbf{f}^{\mathfrak{p}}$, and $\bar{y}^{\mathfrak{p}}$, for each $y \in N \cup \Sigma$, as distinct variables to Var, and consider the following seven B-rules:

$$
\begin{array}{ll}
\frac{\Delta \rightarrow s}{\mathbf{e}^{\mathfrak{p}}, \Delta \rightarrow \mathbf{a}^{\mathfrak{p}}}\left(1_{\mathfrak{p}}\right) & \frac{\Delta, y \rightarrow \mathbf{b}^{\mathfrak{p}}}{\bar{y}^{\mathfrak{p}}, \Delta \rightarrow \mathbf{b}^{\mathfrak{p}}}\left(4_{\mathfrak{p}}\right) \\
\frac{\Delta, y \rightarrow \mathbf{a}^{\mathfrak{p}}}{\bar{y}^{\mathfrak{p}}, \Delta \rightarrow \mathbf{a}^{\mathfrak{p}}}\left(2_{\mathfrak{p}}\right) & \frac{\Delta, \mathbf{e}^{\mathfrak{p}} \rightarrow \mathbf{b}^{\mathfrak{p}}}{\mathbf{f}^{\mathfrak{p}}, \Delta \rightarrow \mathbf{c}^{\mathfrak{p}}}\left(5_{\mathfrak{p}}\right) \\
\frac{\Delta, v_{1}, \ldots, v_{m} \rightarrow \mathbf{a}^{\mathfrak{p}}}{\bar{w}_{1}^{\mathfrak{p}}, \ldots, \bar{w}_{k}^{\mathfrak{p}}, \Delta \rightarrow \mathbf{b}^{\mathfrak{p}}}\left(3_{\mathfrak{p}}\right) & \frac{\Delta, \bar{y}^{\mathfrak{p}} \rightarrow \mathbf{c}^{\mathfrak{p}}}{y, \Delta \rightarrow \mathbf{c}^{\mathfrak{p}}}\left(6_{\mathfrak{p}}\right) \\
\frac{\Delta, \mathbf{f}^{\mathfrak{p}} \rightarrow \mathbf{c}^{\mathfrak{p}}}{\Delta \rightarrow s}\left(7_{\mathfrak{p}}\right) &
\end{array}
$$

By $\mathbf{B}_{\mathcal{G}}$ we denote the set of all B-rules obtained from production rules of $\mathcal{G}$ as shown above; let $\mathcal{B}_{\mathcal{G}}$ be the corresponding set of B-formulae and $\mathbf{B}_{\mathcal{G}}^{\prime}$ be the corresponding set of $\mathrm{B}^{\prime}$-rules.

Before going further, let us comment a bit on these B-rules. In the language without product, we cannot directly implement the 'landing' rule which replaces one subword with another (that is, applies a semi-Thue transition) at an arbitrary place of the antecedent. However, if we manage to move the subword to the right-hand side of the antecedent, it can be indeed replaced by another one (and moved to the left-hand side) by a B-rule, which is our main rule $\left(3_{\mathfrak{p}}\right)$. Other rules do the necessary preparations. This idea is essentially due to Buszkowski; here we present it more straightforwardly. First, $\left(1_{\mathfrak{p}}\right)$ starts the replacement procedure. Second, several applications of $\left(2_{\mathfrak{p}}\right)$ rotate the antecedent so that the necessary subword is on the right-hand side of the antecedent. The usage of an alternative alphabet $\left(\bar{y}^{\mathfrak{p}}\right.$ instead of $\left.y\right)$ and special variables in the succedent $\left(\mathbf{a}^{\mathfrak{p}}, \ldots\right)$ here ensures that this process cannot be aborted, and other rules cannot be applied until we finish. Third, as said above, $\left(3_{\mathfrak{p}}\right)$ performs the actually semi-Thue transition. Finally, $\left(4_{\mathfrak{p}}\right)-\left(7_{\mathfrak{p}}\right)$ perform the backwards rotation and quit the procedure. This strategy is formalized in the proof of the key Lemma 5 below, which is the version of Lemma 1 for encoding Buszkowski's rules.

Lemma 5 Let $\mathcal{L}$ be $\pi_{q}$-sound in $\mathbf{L}^{*}$ and admit the $/ L$ and / $R$ rules (maybe with Lambek's restriction for the latter). Let $\Psi_{\mathcal{G}}$ internalise $\mathcal{B}_{\mathcal{G}}=\left\{B_{1}, \ldots, B_{N}\right\}$ in $\mathcal{L}$ (see Definition 5). Also let all formulae in $\Psi_{\mathcal{G}}$ be !-formulae, for which permutation rules are allowed in $\mathcal{L}$. Then the following are equivalent: 
1. $s \Rightarrow_{\mathcal{G}}^{*} z_{1} \ldots z_{n}$;

2. $z_{1}, \ldots, z_{n} \rightarrow s$ is derivable from axiom $s \rightarrow s$, using only rules from $\mathbf{B}_{\mathcal{G}}$, without cut;

3. $\Psi_{\mathcal{G}}, z_{1}, \ldots, z_{n} \rightarrow s$ is derivable in $\mathcal{L}$.

4. there exists such a bracketing $\Delta$ of $z_{1}, \ldots, z_{n}$ that the sequent $\Psi_{\mathcal{G}}$ is derivable in $\mathcal{L}$;

5. the sequent $! B_{1}, \ldots, ! B_{N}, z_{1}, \ldots, z_{n} \rightarrow s$ is derivable in :MALC* $^{*}$;

6. $z_{1}, \ldots, z_{n} \rightarrow s$ is derivable in $\mathbf{L}^{*}(/)$ extended with rules from $\mathbf{B}_{\mathcal{G}}^{\prime}$.

Proof This proof shares much with the proof of Lemma 1 .

$1 \Rightarrow 2$ Proceed by induction on the derivation of $z_{1} \ldots z_{n}$ from $s$ in $\mathcal{G}$. The base case, $s \Rightarrow_{\mathcal{G}}^{*} s$, corresponds to the $s \rightarrow s$ axiom. For the induction step, consider the last production rule $\mathfrak{p}=\left(v_{1} \ldots v_{m} \Rightarrow w_{1} \ldots w_{k}\right)$ applied in the derivation:

$$
s \Rightarrow_{\mathcal{G}}^{*} z_{1} \ldots z_{i} v_{1} \ldots v_{m} z_{j} \ldots z_{n} \Rightarrow_{\mathcal{G}} z_{1} \ldots z_{i} w_{1} \ldots w_{k} z_{j} \ldots z_{n} .
$$

By induction hypothesis, the sequent $z_{1}, \ldots, z_{i}, v_{1}, \ldots, v_{m}, z_{j}, \ldots, z_{n} \rightarrow s$ is derivable. The necessary sequent $z_{1}, \ldots, z_{i}, w_{1}, \ldots, w_{k}, z_{j}, \ldots, z_{n} \rightarrow s$ is now derived as follows:

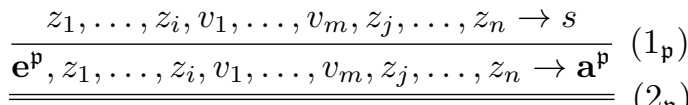

$$
\begin{aligned}
& \overline{\overline{\bar{z}_{j}^{\mathfrak{p}}, \ldots, \bar{z}_{n}^{\mathfrak{p}}, \mathbf{e}^{\mathfrak{p}}, z_{1}, \ldots, z_{i}, v_{1}, \ldots, v_{m} \rightarrow \mathbf{a}^{\mathfrak{p}}}}\left(2_{\mathfrak{p}}\right)
\end{aligned}
$$

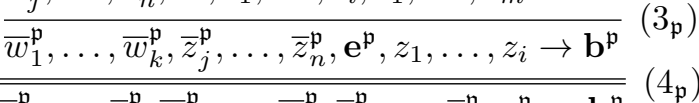

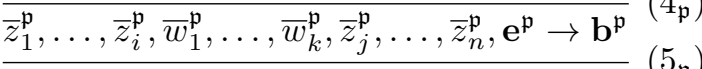

$$
\begin{aligned}
& \overline{\overline{\mathbf{f}^{\mathfrak{p}}, \bar{z}_{1}^{\mathfrak{p}}, \ldots, \bar{z}_{i}^{\mathfrak{p}}, \bar{w}_{1}^{\mathfrak{p}}, \ldots, \bar{w}_{k}^{\mathfrak{p}}, \bar{z}_{j}^{\mathfrak{p}}, \ldots, \bar{z}_{n}^{\mathfrak{p}} \rightarrow \mathbf{c}^{\mathfrak{p}}}}\left(5_{\mathfrak{p}}\right) \\
& \frac{z_{1}, \ldots, z_{i}, w_{1}, \ldots, w_{k}, z_{j}, \ldots, z_{n}, \mathbf{f}^{\mathfrak{p}} \rightarrow \mathbf{c}^{\mathfrak{p}}}{z_{1}, \ldots, z_{i}, w_{1}, \ldots, w_{k}, z_{j}, \ldots, z_{n} \rightarrow s}\left(7_{\mathfrak{p}}\right)
\end{aligned}
$$

$2 \Rightarrow 3$ Proceed by induction on derivation. The base case, $\Phi, s \rightarrow s$, is derivable by item 1 of Definition 5 . The induction step, i.e., application of a B-rule of the form

$$
\frac{\Delta, q_{1}, \ldots, q_{m} \rightarrow r}{p_{1}, \ldots, p_{k}, \Delta \rightarrow t} B
$$

is handled using the 'landing' rule (item 2 of Definition 5) as follows:

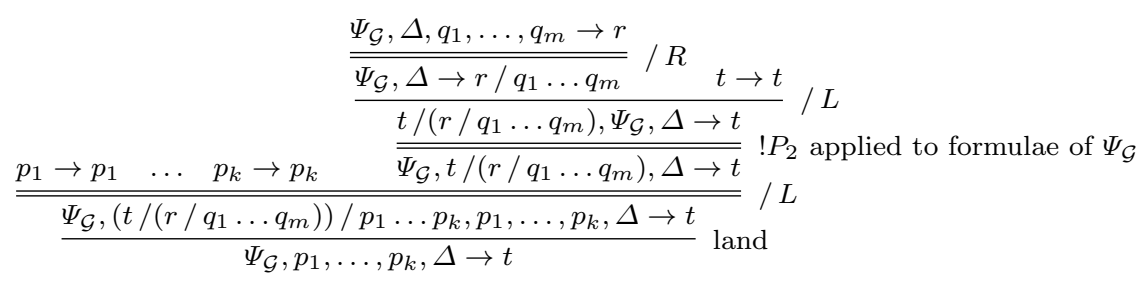

Notice that here we essentially used the permutation rules for formulae of $\Psi_{\mathcal{G}}$. 
$3 \Rightarrow 4$ Obvious: take the trivial (empty) bracketing $\Delta=z_{1}, \ldots, z_{n}$.

$4 \Rightarrow 5$ is handled exactly as in Lemma 1

$5 \Rightarrow 6$ Consider a cut-free derivation of $! B_{1}, \ldots, ! B_{N}, z_{1}, \ldots, z_{n}$ in MALC $^{*}$ and erase all !-formulae from it. Then applications of structural rules for ! become trivial, and $! L$ transforms into

$$
\frac{\Delta_{1}, B, \Delta_{2} \rightarrow A}{\Delta_{1}, \Delta_{2} \rightarrow A}
$$

where $B$ is a $\mathrm{B}$-formula from $\mathcal{B}_{\mathcal{G}}$. This is equivalent to cut with the B-axiom $\Lambda \rightarrow B$.

Thus, $z_{1}, \ldots, z_{n}$ is derivable in $\mathbf{L}^{*}(/)$ extended by the set of $\mathrm{B}$-axioms obtained from $\mathcal{G}$ and, by Lemma 3 , in the corresponding extension by $\mathrm{B}^{\prime}$-rules, $\mathbf{B}_{\mathcal{G}}^{\prime}$.

$6 \Rightarrow 1$ The extension of the Lambek calculus with $\mathbf{B}_{\mathcal{G}}^{\prime}$, admits cut elimination (Lemma 4), and in the cut-free derivation the only rules that can be applied are $\mathrm{B}^{\prime}$-rules.

Proceed by induction on this derivation. The base case is the $s \rightarrow s$ axiom, and we have $s \Rightarrow_{\mathcal{G}}^{*} s$. For the induction step, let us go upwards along the derivation, turning right at each application of a $\mathrm{B}^{\prime}$-rule, and trace the succedent:

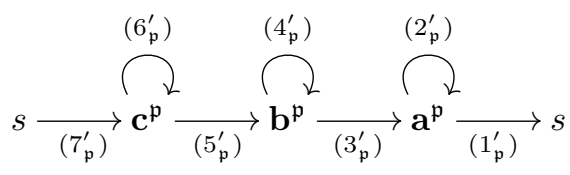

(Since variables $\mathbf{a}^{\mathfrak{p}}, \mathbf{b}^{\mathfrak{p}}$, and $\mathbf{c}^{\mathfrak{p}}$ could never appear in antecedents, the derivation cannot stop at an axiom of the form $\mathbf{a}^{\mathfrak{p}} \rightarrow \mathbf{a}^{\mathfrak{p}}$ or alike.)

Essentially, as we shall see below, once we started with $\left(7_{\mathfrak{p}}^{\prime}\right)$, we fix the production rule $\mathfrak{p}$ and perform, as a whole, the block of $\mathrm{B}^{\prime}$-rules which emulates application of $\mathfrak{p}$ (as the last production rule in the derivation). Then we return to a sequent of the form $\Delta \rightarrow s$, ready to perform our backtracking further.

Variables $\mathbf{d}^{\mathfrak{p}}, \mathbf{e}^{\mathfrak{p}}, \mathbf{f}^{\mathfrak{p}}$, and $\bar{y}^{\mathfrak{p}}(y \in N \cup \Sigma)$ are never succedents of conclusions of rules from $\mathbf{B}_{\mathcal{G}}^{\prime}$. Therefore, left premises of the rules $\left(1_{\mathfrak{p}}^{\prime}\right)-\left(5_{\mathfrak{p}}^{\prime}\right)$, which are of the form $\Pi_{i} \rightarrow p_{i}$, where $p_{i}$ is one of the aforementioned variables, could only be axioms $p_{i} \rightarrow p_{i}$. This means that $\left(1_{\mathfrak{p}}^{\prime}\right)-\left(5_{\mathfrak{p}}^{\prime}\right)$ actually transform into the corresponding B-rules, $\left(1_{\mathfrak{p}}\right)-\left(5_{\mathfrak{p}}\right)$. As for $\left(7_{\mathfrak{p}}^{\prime}\right)$, it already coincides with $\left(7_{\mathfrak{p}}\right)$.

Thus, the bottom of our derivation looks as follows, where $\Delta_{1}, \ldots, \Delta_{n^{\prime}}=$ $z_{1}, \ldots, z_{n}$ : 


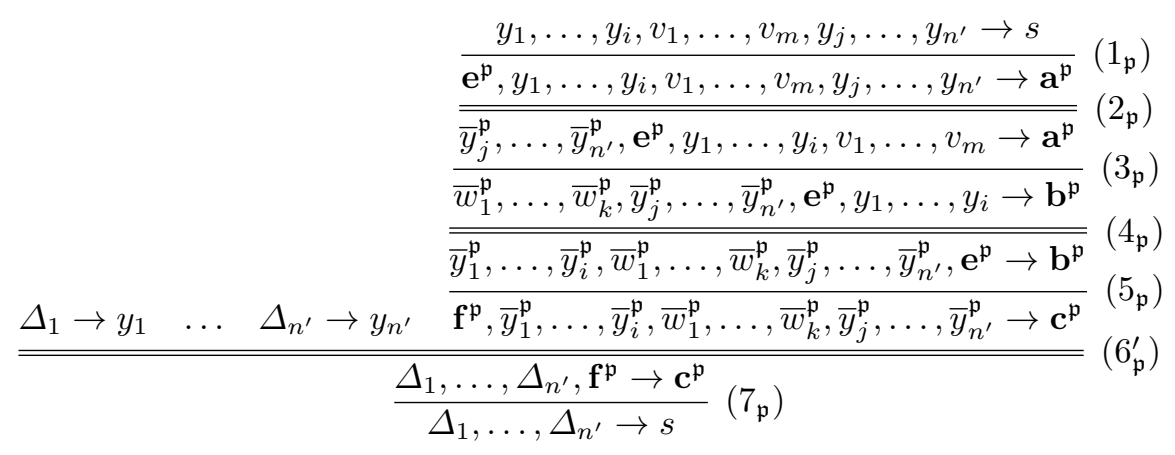

Consider sequents of the form $\Delta_{i} \rightarrow y_{i}$ (left premises); $w_{1}, \ldots, w_{k}$ are also $y$ 's. If $y_{i} \neq s$, then it could not be the succedent of the conclusion of a rule from $\mathbf{B}_{\mathcal{G}}^{\prime}$, therefore $\Delta_{i}=y_{i}$ and this is just an axiom. If $y_{i}=s$, then by induction hypothesis we have $\Delta_{i}$ derivable from $s$ in $\mathcal{G}$. Thus, in both cases ${ }^{3} \mathcal{G}$ derives $\Delta_{i}$ from $y_{i}$.

By induction hypothesis we have $s \Rightarrow_{\mathcal{G}}^{*} y_{1} \ldots y_{i} v_{1} \ldots v_{m} y_{j} \ldots y_{n^{\prime}}$, and since $\mathfrak{p}=\left(v_{1} \ldots v_{m} \Rightarrow w_{1} \ldots w_{k}\right)$ is a production rule of $\mathcal{G}$ (the form of $\mathfrak{p}$ is taken from $\left.\left(3_{\mathfrak{p}}\right)\right), s \Rightarrow_{\mathcal{G}}^{*} y_{1} \ldots y_{i} w_{1} \ldots w_{k} y_{j} \ldots y_{n^{\prime}}$. Finally, we recall a well-known property of derivations in type-0 grammars: if $s \Rightarrow_{\mathcal{G}}^{*} y_{1} \ldots y_{n^{\prime}}\left(w_{i}\right.$ 's are also part of $y_{j}$ 's) and $y_{i} \Rightarrow_{\mathcal{G}}^{*} \Delta_{i}$ for each $i$, then $s \Rightarrow_{\mathcal{G}}^{*} \Delta_{1} \ldots \Delta_{n^{\prime}}=z_{1} \ldots z_{n}$.

This lemma yields results on complexity and generative power of categorial grammars for one-division fragments, exactly as Lemma 1 does in the general case.

Theorem 13 The derivability problems for one-division fragments (that is, fragments including $/, !$, brackets and bracket modalities) of $! \mathbf{L}^{*}, !^{\mathrm{r}} \mathbf{L}^{*}, !_{\mathrm{b}}{ }^{2015} \mathbf{L}^{*} \mathbf{b}$, $!_{\mathrm{b}}^{2015} \mathbf{L}^{*} \mathbf{b}(\mathrm{st})^{\prime}$, and $!_{\mathrm{b}}^{2015} \mathbf{L}^{*} \mathbf{b}(\mathrm{st})$ are undecidable.

Theorem 14 For any r.e. language $M$ and for each of the calculi mentioned in the previous theorem there exists a categorial grammar for $M$ based on the given calculus. For bracketed systems, such a grammar both s-recognises and t-recognises $M$.

For $!_{\mathrm{b}}^{2018} \mathrm{MALC}{ }^{*} \mathrm{~b}(\mathrm{st}){ }^{\prime \prime}$ and $!_{\mathrm{b}}^{2018} \mathrm{MALCb}(\mathrm{st})$, however, we cannot directly use the internalisation given by Proposition 8 , since the meta-formula $\Phi$ used there includes the product connective. Also, $\Phi$ includes $[[q]]$, which is not a !-formula and does not allow permutation, as required in Lemma 5.

We overcome this issue by slightly modifying the notion of internalisation and proving a new version of Proposition 8

\footnotetext{
3 This part can be simplified a bit by modifying $\mathcal{G}$. Namely, we could introduce a new starting symbol $s^{\prime}$ with a rule $s^{\prime} \Rightarrow s$. The language generated by $\mathcal{G}$ will not change. After this transformation, the starting symbol $s^{\prime}$ will never appear in the derivation, except for its start, and therefore there would be always $y_{i} \neq s^{\prime}$, and $\Delta_{i}=y_{i}$.
} 
Definition 6 Let $\mathcal{B}=\left\{B_{1}, \ldots, B_{N}\right\}$ be a finite set of formulae and $\mathcal{V} \subseteq$ Var be a finite set of variables. A meta-formula $\Psi \mathcal{V}$-internalises $\mathcal{B}$ in the calculus $\mathcal{L}$, if the following holds:

1. the sequent $\Psi, s \rightarrow s$ is derivable in $\mathcal{L}$;

2. the following ' $t$-landing' rule is admissible in $\mathcal{L}$ for any $t \in \mathcal{V}$ :

$$
\frac{\Psi, \Delta_{1}, \Delta_{2} \rightarrow t}{\Psi, \Delta_{1}, B_{i}, \Delta_{2} \rightarrow t} \operatorname{land}_{t}, B_{i} \in \mathcal{B}
$$

3. the sequent $! B_{1}, \ldots, ! B_{N} \rightarrow \prod \pi_{q}(\Psi)$ is derivable in $! \mathbf{L}^{*}$.

The new notion of $\mathcal{V}$-internalisation differs from the original notion of internalisation (Definition 5) in item 2. This item is formulated in a weaker form: we restrict the antecedents of sequents in the 'landing' rule by a finite set $\mathcal{V}$ of variables. The key observation is that $\mathcal{V}$-internalisation, where $\mathcal{V}$ is the set of all variables used in $\mathcal{B}$, is already sufficient for Lemma 5 . Thus, now we only have to prove the $\mathcal{V}$-internalisation property for $!_{\mathbf{b}}^{\mathbf{2 0 1 8}} \mathbf{L} * \mathbf{b}(\mathrm{st})^{\prime}$.

Proposition 10 Let $\mathcal{V}=\left\{t_{1}, \ldots, t_{m}\right\}$ be a finite set of variables and $\mathcal{B}=$ $\left\{B_{1}, \ldots, B_{N}\right\}$ be a finite set of Lambek formulae. Then the following metaformula

$\Psi_{\mathcal{B}, \mathcal{V}}=!\left((s / s) / ! Z_{1,1}\right), ! Z_{1,1}, \ldots, !\left((s / s) / ! Z_{m, N}\right), ! Z_{m, N}, !((s / s) /\langle\rangle\langle\rangle q), !\langle\rangle\langle\rangle q$, where

$$
Z_{i, j}=\left([]^{-1}\left(t_{j} /\left(\left(t_{j} / ! B_{i}\right) / !\langle\rangle\langle\rangle q\right)\right)\right) / q,
$$

$\mathcal{V}$-internalises $\mathcal{B}$ in $!_{\mathbf{b}}^{\mathbf{2 0 1 8}} \mathbf{L}^{*} \mathbf{b}$ and in $!_{\mathbf{b}}^{\mathbf{2 0 1 8}} \mathbf{L b}$.

Proof For short, denote $\Psi_{\mathcal{B}, \mathcal{V}}$ by just $\Psi$. Item 1 of Definition 6 is checked exactly as in Proposition 8 .

For item 2, let us check the $t$-landing rule for $t=t_{j} \in \mathcal{V}$ and $B_{i} \in \mathcal{B}$. Let $\Psi=\Psi^{\prime}, !\langle\rangle\langle\rangle q$.

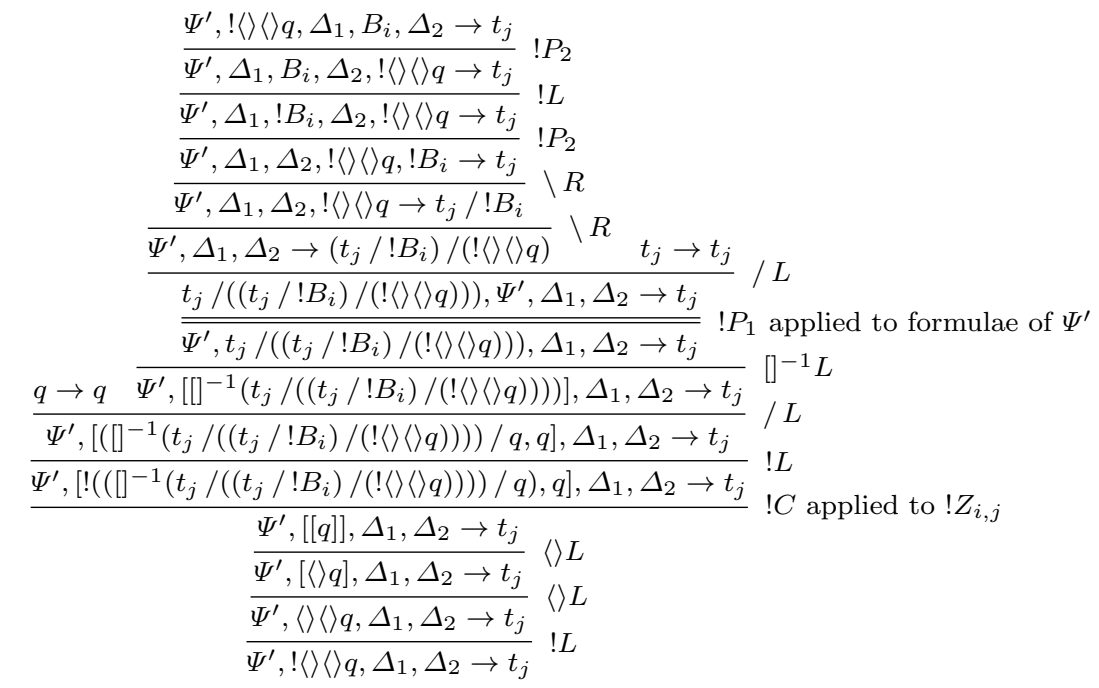


Finally, let us check item 3 . The $\pi_{q}$-projection of $\Psi$ includes the following formulae (the order does not matter due to permutation rules):

1. ! $\left((s / s) / ! \pi_{q}\left(Z_{i, j}\right)\right)$;

2. $\left.! Z_{i, j}=!\left(\left(t_{j} /\left(\left(t_{j} / ! B_{i}\right) / ! \mathbf{1}\right)\right)\right) / \mathbf{1}\right)$;

3. ! $((s / s) / \mathbf{1})$ and $! \mathbf{1}$.

We enjoy the following derivations in $! \mathbf{L}^{*}$ :

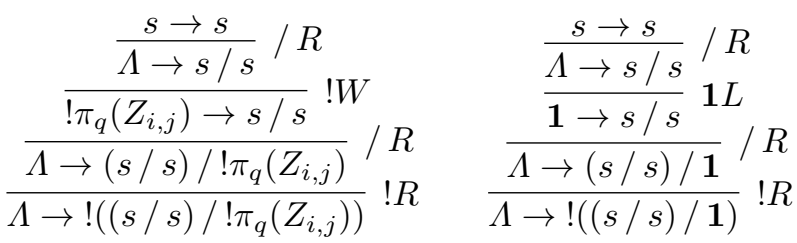

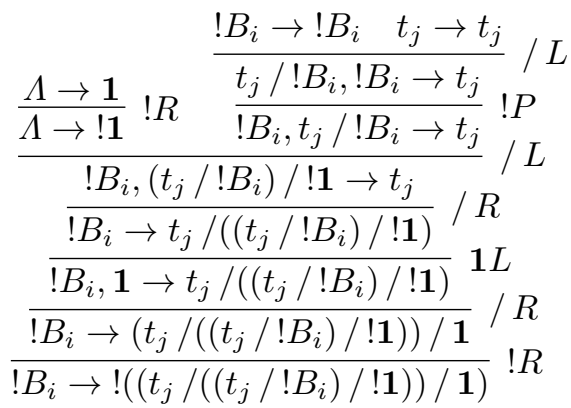

By $\cdot R$, we derive $! B_{1}, \ldots, ! B_{N}, \ldots, ! B_{1}, \ldots, ! B_{N} \rightarrow \prod \pi_{q}(\Psi)\left(\right.$ here $\left.! B_{1}, \ldots, ! B_{N}\right)$ is repeated $m$ times. Permutations and contractions yield the needed sequent $! B_{1}, \ldots, ! B_{N} \rightarrow \prod \pi_{q}(\Psi)$.

By Proposition 1, we propagate this construction to $!_{\mathbf{b}}^{\mathbf{2 0 1 8}} \mathbf{L}^{*} \mathbf{b}(\mathrm{st})^{\prime}$ and $!_{b}^{2018} \mathbf{L b}(\mathrm{st})^{\prime}$. Finally, for the original Morrill's system $!_{\mathbf{b}}^{\mathbf{2 0 1 8}} \mathbf{L}^{*} \mathbf{b}(\mathrm{st})$ we use the same trick as in Section 9 (Proposition 9), adding an extra ! over $Z_{i, j}$. In a whole, this yields the necessary results.

Theorem 15 The derivability problems for one-division fragments (that is, fragments including $/$, !, brackets and bracket modalities) of $!_{\mathbf{b}}^{\mathbf{2 0 1 8}} \mathbf{L}^{*} \mathbf{b}, !_{\mathbf{b}}^{\mathbf{2 0 1 8}} \mathbf{L b}$, $!_{\mathrm{b}}^{\mathbf{2 0 1 8}} \mathbf{L}^{*} \mathbf{b}(\mathrm{st})^{\prime}, !_{\mathrm{b}}^{\mathbf{2 0 1 8}} \mathbf{L b}(\mathrm{st})^{\prime}$, and $!_{\mathrm{b}}^{\mathbf{2 0 1 8}} \mathbf{L}^{*} \mathbf{b}(\mathrm{st})$ are undecidable.

Theorem 16 For any r.e. language $M$ and for each of the calculi mentioned in the previous theorem there exists a categorial grammar based on the given calculus which both s-recognises and t-recognises $M$, for calculi without Lambek's restriction $\left(!_{\mathbf{b}}^{\mathbf{2 0 1 8}} \mathbf{L}^{*} \mathbf{b}, !_{\mathbf{b}}^{\mathbf{2 0 1 8}} \mathbf{L}^{*} \mathbf{b}(\mathrm{st})^{\prime}\right.$, and $\left.!_{\mathbf{b}}^{\mathbf{2 0 1 8}} \mathbf{L}^{*} \mathbf{b}(\mathrm{st})\right)$, and $M-\{\varepsilon\}$, in the case with Lambek's restriction $\left(!_{\mathbf{b}}^{\mathbf{2 0 1 8}} \mathbf{L b}\right.$ and $\left.!_{\mathbf{b}}^{\mathbf{2 0 1 8}} \mathbf{L b}(\mathrm{st})^{\prime}\right)$. 


\section{Conclusions and Future Work}

In this article, we have performed the logical analysis of two systems introduced by Morrill as a base for the CatLog categorial grammar parser, $!_{\mathrm{b}}^{\mathbf{2 0 1 5}} \mathbf{M A L C} \mathbf{C}^{*} \mathbf{b}(\mathrm{st})$ and $!_{\mathrm{b}}^{2018} \mathbf{M A L C}{ }^{*} \mathbf{b}(\mathrm{st})$. We have pointed out issues with cut elimination in these systems, and provided necessary modification for which cut elimination is proved. We also discussed how Lambek's non-emptiness restriction can be imposed on $!_{b}^{\mathbf{2 0 1 8}} \mathbf{M A L C}{ }^{*} \mathbf{b}(\mathrm{st})$. From the algorithmic point of view, we have proved undecidability for each of Morrill's systems, even in the smallest possible language with only one division, brackets and bracket modalities, and the subexponential. Moreover, we have shown that categorial grammars based on Morrill's calculi can generate arbitrary recursively enumerable languages (in the case with Lambek's restriction - arbitrary r.e. languages without the empty word).

One of the most interesting questions for future research is as follows. The undecidability results presented in this article look unfortunate, since the calculi it is applied to are intended to be used in natural language parsing software.

Thus, it is an important task to explore fragments of the calculi, guarded by certain syntactic conditions on applying !, for which the derivability problem is decidable. For systems without brackets, in particular, ! ${ }^{\mathbf{r}} \mathbf{M} \mathbf{A L C} \mathbf{L}^{*}$, such an algorithm exists under the condition that ! is applied only to variables (Kanovich et al. 2016b). The complexity of this algorithm is the same as for the calculus without !: NP for ! ${ }^{\mathrm{r}} \mathbf{L}^{*}$ and PSPACE for ${ }^{\mathrm{r}} \mathbf{M A L C} \mathbf{C}^{*}$. Moreover, for ! $\mathbf{L}^{*}$ decidability is known for a broader class of formulae allowed under !, namely, formulae of implication depth 1 , that is, of the form $p_{1} \ldots p_{k} \backslash q / r_{1} \ldots r_{m}$ (Fofanova, 2018). Extending this result to !MALC ${ }^{*}$ and ! $^{\mathbf{r}} \mathbf{M} \mathbf{A L C} \mathbf{C}^{*}$ is still an open question.

For systems with brackets, the class of formulae for which the derivability problem becomes decidable appears to be much broader. For Morrill's first system, $!_{\mathbf{b}}^{\mathbf{2 0 1 5}} \mathbf{M A L C} \mathbf{C}^{*} \mathbf{b}(\mathrm{st})$, this class is guarded by so-called bracket nonnegative condition (BNNC) imposed on !-formulae. Under this condition, ! can be applied to any formula which does not include negative occurrences of \langle\rangle and does not include positive occurrences of [ $]^{-1}$. In particular, ! is allowed to be applied to any formula which does not include bracket modalities at all, no matter how complex this formula is. Morrill and Valentín (2015a) show that the derivability problem in $\mathbf{!}_{\mathbf{b}}^{\mathbf{2 0 1 5}} \mathbf{M} \mathbf{A L} \mathbf{C}^{*} \mathbf{b}$ (st) for sequents obeying BNNC is decidable; Kanovich et al. (2017b) establish an NP upper complexity bound for its fragment without additives, also with BNNC imposed. We conjecture that for the full system, including additives, the complexity bound is PSPACE. These complexity boundaries are tight, since the multiplicative-only Lambek calculus is already NP-complete (Pentus, 2006) and MALC* is PSPACEcomplete (Kanovich, 1994). For Morrill's second system, $!_{\mathbf{b}}^{\mathbf{2 0 1 8}}{ }^{\mathbf{M A L C}}{ }^{*} \mathbf{b}$ (st), formulating the corresponding version of BNNC and proving decidability for the fragment guarded by this new condition is a problem for further investigation. 
Another, potentially simpler but more technical question left for further research is the question of extending our cut elimination proof to calculi with discontinuous operations (Morrill and Valentín, 2010). We conjecture that the proof could be obtained as a combination of our proof (using "deep cut elimination" for !-formulae) presented here and the proof by Morrill and Valentín (2010) for displacement calculus. The notations, however, would become extremely complicated - thus, a digestable presentation of such a proof becomes a separate challenge.

Acknowledgements. We are grateful to Glyn Morrill for a number of very helpful interactions we benefited from at various stages of this work. The work of Max Kanovich was partially supported by EPSRC Programme Grant EP/R006865/1: "Interface Reasoning for Interacting Systems (IRIS)." The work of Andre Scedrov and Stepan Kuznetsov was prepared within the framework of the HSE University Basic Research Program and partially funded by the Russian Academic Excellence Project ' $5-100$.' The work of Stepan Kuznetsov was also partially supported by the Council of the President of Russia for Support of Young Russian Researchers and Leading Research Schools of the Russian Federation, by the Young Russian Mathematics Award, and by the Russian Foundation for Basic Research grant 20-01-00435.

\section{References}

Abrusci, V. M. (1990). A comparison between Lambek syntactic calculus and intuitionistic linear logic. Zeitschr. math. Logik Grundl. Math. (Math. Logic Q.), 36:11-15.

Ajdukiewicz, K. (1935). Die syntaktische Konnexität. Studia Philosophica, $1: 1-27$.

Andreoli, J.-M. (1992). Logic programming with focusing proofs in linear logic. J. Logic Comput., 2(3):297-347.

Bar-Hillel, Y. (1953). A quasi-arithmetical notation for syntactic description. Language, 29:47-58.

Bar-Hillel, Y., Gaifman, C., \& Shamir, E. (1960). On the categorial and phrase-structure grammars. Bull. Res. Council Israel, 9F:1-16.

Braüner, T. (2000). A cut-free Gentzen formulation of the modal logic S5. Log. J. IGPL, 8(5):629-643.

Braüner, T. \& de Paiva, V. (1996). Cut elimination for full intuitionistic linear logic. BRICS Report RS-96-10.

Braüner, T. \& de Paiva, V. (1998). A formulation of linear logic based on dependency relations. In: CSL 1997: Computer Science Logic, M. Nielsen $\&$ W. Thomas, ed., volume 1414 of $L N C S$, pages 129-148. Springer.

Buszkowski, W. (1982). Some decision problems in the theory of syntactic categories. Zeitschr. Math. Logik Grundl. Math. (Math. Logic Q.), 28:539548 . 
Buszkowski, W. (1985). The equivalence of unidirectional lambek categorial grammars and context-free grammars. Zeitschr. Math. Logik Grundl. Math. (Math. Logic Q.), 31:369-384.

Buszkowski, W. (2003). Type logics in grammar. In: Trends in Logic: 50 Years of Studia Logica, pages 337-382. Springer.

Buszkowski, W. (2005). Lambek calculus with nonlogical axioms. In: Language and Grammar, volume 168 of CSLI Lect. Notes, pages 77-93. CSLI.

Buszkowski, W. \& Palka, E. (2008). Infinitary action logic: complexity, models and grammars. Studia Logica, 89:1-18.

Carpenter, B. (1997). Type-logical semantics. MIT Press.

Chomsky, N. (1956). Three models for the description of language. IRE Trans. Inform. Theory, I T-2(3):113-124.

Dekhtyar, M. \& Dikovsky, A. (2008). Generalized categorial dependency grammars. In: Pillars of Computer Science: Trakhtenbrot/Festschrift, A. Avron, N. Dershowitz, \& A. Rabinovich, ed., volume 4800 of LNCS, pages 230-255. Springer.

Eades III, H. \& de Paiva, V. (2020). Multiple conclusion linear logic: cut elimination and more. J. Logic Comput., 30(1):157-174.

Fadda, M. \& Morrill, G. (2005). The Lambek calculus with brackets. In: Language and Grammar: Studies in Mathematical Linguistics and Natural Language, pages 113-128. CSLI.

Fofanova, E. M. (2018). Algorithmic decidability of a fragment of the Lambek calculus with an exponential modality. In: Mal'tsev Meeting 2018, Collection of Abstracts. Sobolev Inst. Math. \& Novosibirsk State University. (in Russian).

Gentzen, G. (1935). Untersuchungen über das logische Schließen I. Mathematische Zeitschrift, 39:176-210.

Girard, J.-Y. (1987). Linear logic. Theoretical Computer Science, 50(1):1-102.

Girard, J.-Y. (1991). A new constructive logic: classical logic. Mathematical Structures in Computer Science, 1(3):255-296.

Girard, J.-Y. (1993). On the unity of logic. Annals of Pure and Applied Logic, 59(3):201-217.

Greibach, S. A. (1965). A new normal-form theorem for context-free phrase structure grammars. J. ACM, 12(1):42-52.

Hodas, J. \& Miller, D. (1994). Logic programming in a fragment of intuitionistic linear logic. Information and Computation, 110(2):327-365.

Jäger, G. (2003). On the generative capacity of multi-modal categorial grammars. Research on Language and Computation, 1(1-2):105-125.

Kanazawa, M. (1992). The Lambek calculus enriched with additional connectives. J. Log. Lang. Inform., 1(2):141-171.

Kanazawa, M. (1999). Lambek calculus: Recognizing power and complexity. In: JFAK. Essays dedicated to Johan van Benthem to the occasion of his 50th birthday. Vossiuspers, Amsterdam Univ. Press.

Kanazawa, M. (2018). On the recognizing power of the Lambek calculus with brackets. J. Log. Lang. Inform., 27(4):295-312. 
Kanovich, M. (1994). Horn fragments of non-commutative logics with additives are PSPACE-complete. Extended abstract, presented at CSL '94, Kazimierz, Poland.

Kanovich, M., Kuznetsov, S., Morrill, G., \& Scedrov, A. (2017a). A polynomial-time algorithm for the Lambek calculus with brackets of bounded order. In: 2nd International Conference on Formal Structures for Computation and Deduction (FSCD 2017), D. Miller, ed., volume 84 of Leibniz International Proceedings in Informatics (LIPIcs), pages 22:122:17. Schloss Dagstuhl-Leibniz-Zentrum für Informatik.

Kanovich, M., Kuznetsov, S., Nigam, V., \& Scedrov, A. (2018). A logical framework with commutative and non-commutative subexponentials. In: IJCAR 2018: Automated Reasoning, volume 10900 of LNAI, pages 228-245. Springer.

Kanovich, M., Kuznetsov, S., Nigam, V., \& Scedrov, A. (2019a). Subexponentials in non-commutative linear logic. Math. Struct. Comput. Sci., 29(8):1217-1249.

Kanovich, M., Kuznetsov, S., \& Scedrov, A. (2016a). On Lambek's restriction in the presence of exponential modalities. In: Proc. LFCS 2016, S. Artemov \& A. Nerode, ed., volume 9537 of LNCS, pages 146-158. Springer.

Kanovich, M., Kuznetsov, S., \& Scedrov, A. (2016b). Undecidability of the Lambek calculus with a relevant modality. In: FG 2015, FG 2016: Formal Grammar, A. Foret, G. Morrill, R. Muskens, R. Osswald, \& S. Pogodalla, ed., volume 9804 of $L N C S$, pages 240-256. Springer.

Kanovich, M., Kuznetsov, S., \& Scedrov, A. (2017b). Undecidability of the Lambek calculus with subexponential and bracket modalities. In: FCT 201\%: Fundamentals of Computation Theory, R. Klasing \& M. Zeitoun, ed., volume 10472 of $L N C S$, pages 326-340. Springer.

Kanovich, M., Kuznetsov, S., \& Scedrov, A. (2019b). Undecidability of a newly proposed calculus for CatLog3. In: FG 2019: Formal Grammar, R. Bernardi, G. Kobele, \& S. Pogodalla, ed., volume 11668 of LNCS, pages 67-83. Springer.

Kanovich, M., Kuznetsov, S., \& Scedrov, A. (2020). Reconciling Lambek's restriction, cut-elimination, and substitution in the presence of exponential modalities. J. Logic Comput., 30(1):239-256.

Kozen, D. (2002). On the complexity of reasoning in Kleene algebra. Inform. Comput., 179:152-162.

Kuznetsov, S. (2012a). Lambek grammars with one division and one primitive type. Log. J. IGPL, 20(1):207-221.

Kuznetsov, S. (2012b). Lambek grammars with the unit. In: $F G$ 2010, FG 2011: Formal Grammar, P. de Groote \& M.-J. Nederhof, ed., volume 7395 of $L N C S$, pages 262-266. Springer.

Kuznetsov, S. (2013). Conjunctive grammars in Greibach normal form and the Lambek calculus with additive connectives. In: FG 2013, FG 2012: Formal Grammar, G. Morrill \& M.-J. Nederhof, ed., volume 8036 of LNCS, pages 242-249. Springer. 
Kuznetsov, S. (2017). The Lambek calculus with iteration: two variants. In: WoLLIC 2017: Logic, Language, Information, and Computation, J. Kennedy \& R. J. G. B. de Queiroz, ed., volume 10388 of LNCS, pages 182-198. Springer.

Kuznetsov, S. (2019). The logic of action lattices is undecidable. In: Proc. 34th Annual ACM/IEEE Symposium on Logic in Computer Science (LICS 2019). IEEE.

Kuznetsov, S. \& Okhotin, A. (2017). Conjunctive categorial grammars. In: Proc. MoL '17, volume W17-3414 of ACL Anthology, pages 140-151.

Kuznetsov, S. L. (2016). On translating Lambek grammars with one division into context-free grammars. Proc. Steklov Inst. Math., 294:129-138.

Lambek, J. (1958). The mathematics of sentence structure. Amer. Math. Monthly, 65:154-170.

Lincoln, P., Mitchell, J., Scedrov, A., \& Shankar, N. (1992). Decision problems for propositional linear logic. Annals of Pure and Applied Logic, 56(1):239 311.

Markov, A. (1947). On the impossibility of certain algorithms in the theory of associative systems. Doklady Acad. Sci. USSR (N. S.), 55:583-586.

Moortgat, M. (1996). Multimodal linguistic inference. J. Log. Lang. Inform, $5(3-4): 349-385$.

Moot, R. \& Retoré, C. (2012). The logic of categorial grammars: a deductive account of natural language syntax and semantics, volume 6850 of LNCS. Springer.

Morrill, G. (1992). Categorial formalisation of relativisation: pied piping, islands, and extraction sites. Technical Report LSI-92-23-R, Universitat Politècnica de Catalunya.

Morrill, G. (2014). A categorial type logic. In: Categories and Types in Logic, Language, and Physics: Essays Dedicated to J. Lambek on the Occasion of His 90th Birthday, C. Casadio, B. Coecke, M. Moortgat, \& P. Scott, ed., LNCS, pages 331-352. Springer.

Morrill, G. (2017a). Grammar logicised: relativisation. Linguistics and Philosophy, 40(2):119-163.

Morrill, G. (2017b). Parsing logical grammar: CatLog3. In: Proc. LACompLing2017, pages 107-131.

Morrill, G. (2018a). The CatLog3 technical manual. Technical report, Universitat Politècnica de Catalunya. http://www.lsi.upc.edu/ ${ }^{\sim}$ morrill/CatLog3/CatLog3.pdf.

Morrill, G. (2018b). A note on movement in logical grammar. J. Lang. Model., 6(2):353-363.

Morrill, G. (2019). Parsing/theorem-proving for logical grammar CatLog3. J. Log. Lang. Inform., page 34 pp. (forthcoming).

Morrill, G., Kuznetsov, S., Kanovich, M., \& Scedrov, A. (2018). Bracket induction for Lambek calculus with bracket modalities. In: FG 2018: Formal Grammar, A. Foret, G. Kobele, \& S. Pogodalla, ed., volume 10950 of LNCS, pages 84-101. Springer. 
Morrill, G. \& Valentín, O. (2010). Displacement calculus. Linguistic Analysis, 36(1-4):167-192.

Morrill, G. \& Valentín, O. (2015a). Computation coverage of TLG: Nonlinearity. In: Proc. NLCS '15, volume 32 of EPiC Series, pages 51-63.

Morrill, G. \& Valentín, O. (2015b). Multiplicative-additive focusing for parsing as deduction. In: First International Workshop on Focusing (affiliated with LPAR), Suva, Fiji, volume 197 of EPTCS, pages 29-54.

Morrill, G. V. (2011). Categorial grammar: logical syntax, semantics, and processing. Oxford Univ. Press.

Okhotin, A. (2013). Conjunctive and boolean grammars: the true general case of the context-free grammars. Comput. Sci. Review, 9:27-59.

Pentus, M. (1993). Lambek grammars are context-free. In: Proc. LICS '93, pages 429-433. IEEE.

Pentus, M. (2006). Lambek calculus is NP-complete. Theor. Comput. Sci., 357(1):186-201.

Post, E. L. (1947). Recursive unsolvability of a problem of Thue. J. Symb. Log., 12:1-11.

Pullum, G. K. \& Gazdar, G. (1982). Natural languages and context-free languages. Linguistics and Philosophy, 4(4):471-504.

Safiullin, A. N. (2007). Derivability of admissible rules with simple premises in the Lambek calculus. Moscow Univ. Math. Bull., 62(4):168-171.

Savateev, Y. (2010). Unidirectional Lambek grammars in polynomial time. Theory Comput. Syst., 46(4):662-672.

Shieber, S. M. (1985). Evidence against the context-freeness of natural languages. Linguistics and Philosophy, 8:333-343.

Steedman, M. (2000). The syntactic process. MIT Press.

Thue, A. (1914). Probleme über Veränderungen von Zeichenreihen nach gegebener Regeln. Kra. Vidensk. Selsk. Skrifter., 10.

Versmissen, K. (1996). Grammatical composition: modes, models, modalities. PhD thesis, OTS Utrecht. 